\title{
Conversions among Several Classes of Predicate Encryption and Applications to ABE with Various Compactness Tradeoffs
}

\author{
Nuttapong Attrapadung*1, Goichiro Hanaoka ${ }^{\dagger 1}$, and Shota Yamada ${ }^{\ddagger 1}$ \\ ${ }^{1}$ National Institute of Advanced Industrial Science and Technology (AIST).
}

July 13,2015

\begin{abstract}
Predicate encryption is an advanced form of public-key encryption that yield high flexibility in terms of access control. In the literature, many predicate encryption schemes have been proposed such as fuzzy-IBE, KP-ABE, CP-ABE, (doubly) spatial encryption (DSE), and ABE for arithmetic span programs. In this paper, we study relations among them and show that some of them are in fact equivalent by giving conversions among them. More specifically, our main contributions are as follows:

- We show that monotonic, small universe KP-ABE (CP-ABE) with bounds on the size of attribute sets and span programs (or linear secret sharing matrix) can be converted into DSE. Furthermore, we show that DSE implies non-monotonic CP-ABE (and KP-ABE) with the same bounds on parameters. This implies that monotonic/non-monotonic KP/CP-ABE (with the bounds) and DSE are all equivalent in the sense that one implies another.

- We also show that if we start from KP-ABE without bounds on the size of span programs (but bounds on the size of attribute sets), we can obtain $A B E$ for arithmetic span programs. The other direction is also shown: ABE for arithmetic span programs can be converted into KP-ABE. These results imply, somewhat surprisingly, KP-ABE without bounds on span program sizes is in fact equivalent to $\mathrm{ABE}$ for arithmetic span programs, which was thought to be more expressive or at least incomparable.

By applying these conversions to existing schemes, we obtain many non-trivial consequences. We obtain the first non-monotonic, large universe $\mathrm{CP}-\mathrm{ABE}$ (that supports span programs) with constantsize ciphertexts, the first KP-ABE with constant-size private keys, the first (adaptively-secure, multiuse) $\mathrm{ABE}$ for arithmetic span programs with constant-size ciphertexts, and more. We also obtain the first attribute-based signature scheme that supports non-monotone span programs and achieves constant-size signatures via our techniques.
\end{abstract}

Keywords. Attribute-based encryption, doubly spatial encryption, generic conversion, constant-size ciphertexts, constant-size keys, arithmetic span programs

\footnotetext{
${ }^{*}$ n. attrapadungeaist.go.jp

thanaoka-goichirolaist.go.jp

${ }^{\ddagger}$ yamada-shota@aist.go.jp
} 


\section{Introduction}

Predicate encryption (PE) is an advanced form of public-key encryption that allows much flexibility. Instead of encrypting data to a target recipient, a sender will specify in a more general way about who should be able to view the message. In predicate encryption for a predicate $R$, a sender can associate a ciphertext with a ciphertext attribute $Y$ while a private key is associated with a key attribute $X$. Such a ciphertext can then be decrypted by such a key if the predicate evaluation $R(X, Y)$ holds true.

There exist many classes of PE, each is defined by specifying a corresponding class of predicates. One notable class is attribute-based encryption (ABE) [40,25] for span programs (or equivalently, linear secret sharing schemes), of which predicate is defined over key attributes being a span program and ciphertext attributes being a set of attributes, and its evaluation holds true if the span program accepts the set. This is called key-policy ABE (KP-ABE). There is also ciphertext-policy ABE (CP-ABE), where the roles of key and ciphertext attributes are exchanged. Another important class is doubly spatial encryption (DSE) [26], of which predicate is defined over both key and ciphertext attributes being affine subspaces, and its evaluation holds true if both subspaces intersect. Very recently, a new important class of PE, that is called attribute encryption for arithmetic span programs is defined in [29]. They showed such a PE scheme is useful by demonstrating that the scheme can be efficiently converted into ABE for arithmetic branching programs for both zero-type and non-zero type predicates. If the scheme satisfies a certain requirement for efficiency (namely, encryption cost is at most linear in ciphertext predicate size), it is also possible to obtain a publicly verifiable delegation scheme for arithmetic branching programs, by exploiting a conversion shown in [38]. Furthermore, they gave a concrete construction of such scheme.

Compared to specific constructions of predicate encryption [31, 32, 34, 44, 22, 20] (to name just a few) that focus on achieving more expressive predicates and/or stronger security guarantee, relations among predicate encryption schemes are much less investigated. The purpose of this paper is to improve our understanding of relations among them.

\subsection{Our Results}

Relations among PE. Towards the goal above, we study relations among PE and show that some of them are in fact equivalent by giving generic conversion among them. We first investigate the relation among $\mathrm{ABE}$ with some bounds on parameters (the size of attribute sets and the size of span programs) and DSE. We have the following results:

- First, we show a conversion from KP-ABE (or CP-ABE) with the bounds on parameters into DSE (without key delegation, in Section 3). Such an implication is not straightforward in the first place. Intuitively, one reason stems from the different nature between both predicates: while DSE can be considered as an algebraic object that involves affine spaces, ABE can be seen as a somewhat more combinatorial object that involves sets (of attributes). Our approach involves some new technique for "programming" a set associated to a ciphertext and a span program associated to a private key in the $\mathrm{KP}-\mathrm{ABE}$ scheme so that they can emulate the relation for doubly spatial encryption.

- We then extend the result of [26], which showed that DSE implies CP/KP-ABE with large universes. We provide a new conversion from DSE (without delegation) to non-monotonic CP/KP-ABE with large universes (in Section 4). We note that the resulting schemes obtained by the above conversions have some bounds on parameters. In the conversion, we extensively use a special form of polynomial introduced in [30] and carefully design a matrix so that DSE can capture a relation for ABE.

Somewhat surprisingly, by combining the above results, we obtain generic conversions that can boost the functionality of (bounded) ABE: from monotonic to non-monotonic, and from small-universe to large- 


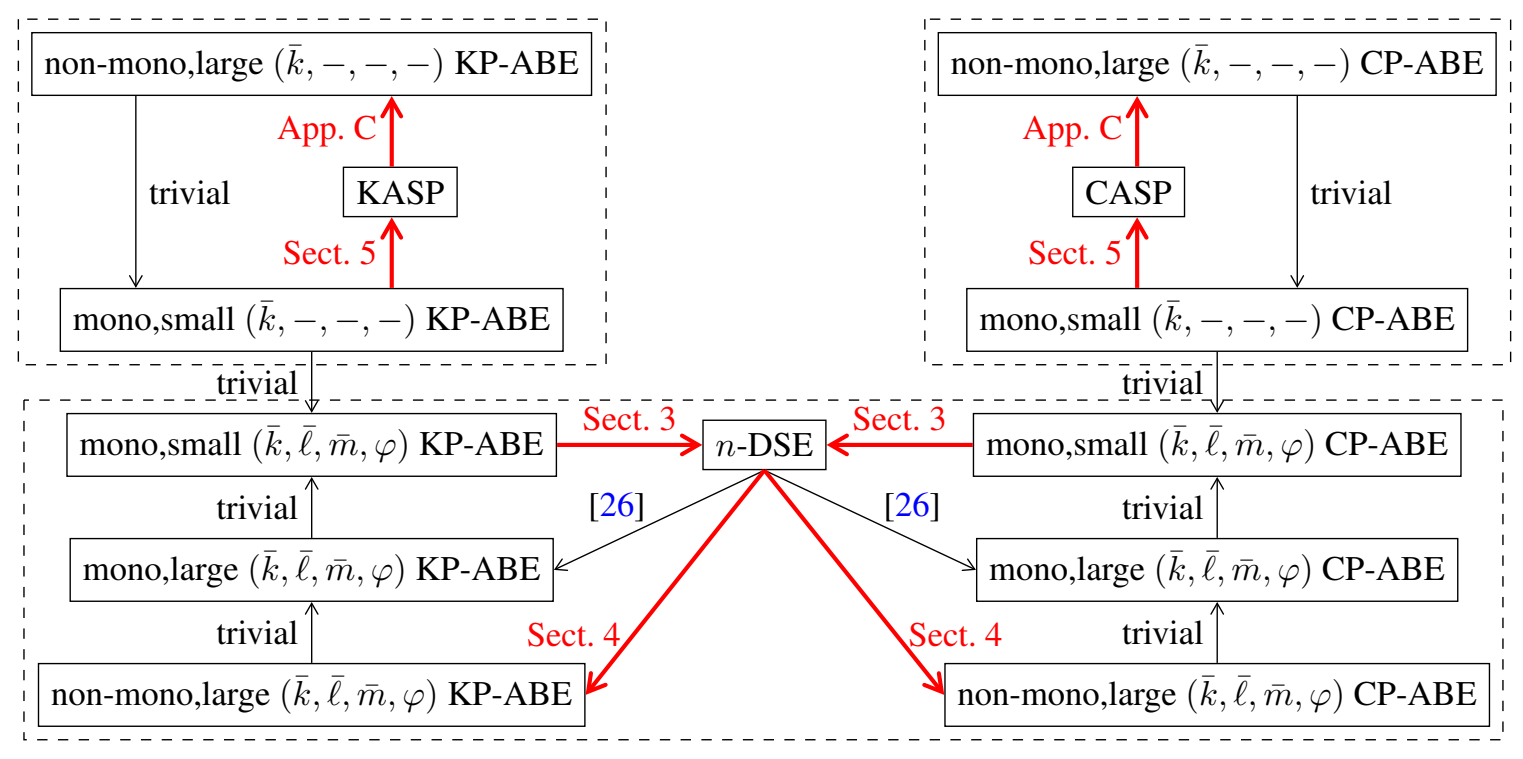

Figure 1: Relations among predicate encryption primitives. In this figure, arrows indicate conversions that transform the primitive of the starting point to that of the end point. The red arrows indicate our results in this paper. For ABE, 'mono' and 'non-mono' indicates whether it is monotonic or non-monotonic, while 'small' and 'large' indicate whether the attribute universes are large (i.e., exponentially large) or small (i.e., polynomially bounded). $(\bar{k}, \bar{\ell}, \bar{m}, \varphi)$ specify bounds on size of sets of attributes and span programs. See Section 2.1 for details. As a result, primitives inside each dashed box are all equivalent in the sense there is a conversion between each pair.

universe; moreover, we also obtain conversions which transform ABE to its dual (key-policy to ciphertextpolicy, and vice versa). This implies that they are essentially equivalent in some sense. See Figure 1 for the details.

So far, we have considered ABE schemes with bounds on parameters, especially on the size of span programs. We then proceed to investigate relation among ABE schemes without bounds on the size of span programs (but with a bound on the size of attribute sets) and ABE for arithmetic span programs recently introduced and studied by Ishai and Wee [29]. We call the latter key-policy ABE for arithmetic span programs (KASP), since in the latter, a ciphertext is associated with a vector while a private key is associated with an arithmetic span program which specifies a policy. By exchanging key and ciphertext attribute, we can also define ciphertext-policy version of ABE for arithmetic span program (CASP). We have the following results:

- We show that monotonic KP-ABE with small universe (without bound on the size of span programs) can be converted into KASP (in Section 5). The idea for the conversion is similar to that in Section 3.

- We then proceed to investigate the converse direction. In fact, we can show somewhat stronger result. That is, we show that KASP can be converted into non-monotonic KP-ABE with large universe, which trivially implies monotonic KP-ABE with small universe (in Appendix C). The idea for the conversion is similar to that in Section 4.

Given the above results, we have all of the following are equivalent: monotonic KP-ABE with small universe, non-monotonic KP-ABE with large universe, and KASP. Similar implications hold for the case of CP-ABE and CASP. However, we do not have a conversion from KP-ABE to CP-ABE in this case. Again, see Figure 1 for the details. 
Direct Applications: New Instantiations. By applying our conversions to existing schemes, we obtain many new instantiations. Most of them have new properties that were not achieved before. These include

- the first DSE with constant-size public key,

- the first DSE with constant-size ciphertexts,

- the first DSE with constant-size private keys,

- the first non-monotonic, large-universe CP-ABE with constant-size ciphertexts,

- the first non-monotonic, large-universe KP-ABE with constant-size keys,

- the first KASP, CASP with constant-size public key,

- the first KASP, CASP with adaptive security and unbounded multi-use,

- the first KASP with constant-size ciphertexts,

- the first CASP with constant-size keys,

which together offer various compactness tradeoffs. Previously, all DSE schemes require linear (or more) sizes in all parameters [26, 17, 14]. Previous CP-ABE with constant-size ciphertexts $[19,13,21,12]$ can only deal with threshold or even more limited expressiveness. As for KP-ABE, to the best of our knowledge, there were no constructions with constant-size keys. ${ }^{1}$ Previous KASP and CASP [29, 16] require linear sizes in all parameters. Moreover, the adaptively secure schemes [16] support only attribute one-use. See Section 6 and tables therein for our instantiations and comparisons. Note also that while our conversion to DSE does not explicitly support key delegation algorithms, we provide them additionally in Appendix B.

Application to Attribute-Based Signatures. Our technique is also useful in the settings of attributebased signatures (ABS) [35, 36]. We first define a notion that we call predicate signature (PS) which is a signature analogue of PE. Then, we construct a specific PS scheme with constant-size signatures such that a signature is associated with a set of attributes while a private key is associated with a policy (or monotone span programs). This is in some sense a dual notion of ordinary ABS in which a signature is associated with a policy and a private key with a set. By using the technique developed in the above, we can convert the PS scheme into an ABS scheme. As a result, we obtain the first ABS scheme with constantsize signatures. Previous ABS schemes with constant-size signatures [27, 12] only support threshold or more limited policies.

Finally, we remark that although our conversions are feasible, they often introduce polynomial-size overheads to some parameters. Thus, in most cases, above schemes obtained by the conversions should be seen as feasibility results in the sense that they might not be totally efficient.

\subsection{Related Works}

There are several previous works investigating relations among PE primitives. In [24], a black box separation between threshold predicate encryption (fuzzy IBE) and IBE was shown. They also rule out certain natural constructions of $\mathrm{PE}$ for $\mathbf{N C}^{1}$ from $\mathrm{PE}$ for $\mathbf{A C}^{0}$. In [15], it was shown that hierarchical inner product encryption is equivalent to spatial encryption, which is a special case of doubly spatial encryption.

[23] showed a generic conversion from KP-ABE supporting threshold formulae to CP-ABE supporting threshold formulae. Their result and ours are incomparable. Our KP-ABE to CP-ABE conversion requires the original KP-ABE to support monotone span programs, which is a stronger requirement than [23]. On the other hand, the resulting scheme obtained by our conversion supports non-monotone span programs, which is a wider class than threshold formulae ${ }^{2}$. Thus, by applying our conversion, we can obtain new

\footnotetext{
${ }^{1} \mathrm{KP}-\mathrm{ABE}$ with (asymptotically) short keys was also proposed in [9]. Compared to ours, their key size is not constant but they focus on more expressive $A B E$, namely $A B E$ for circuits.

${ }^{2}$ While it is known that monotone span programs contain threshold formulae [25], the converse is not known to be true.
} 
schemes (such as $\mathrm{CP}-\mathrm{ABE}$ supporting non-monotone span programs with constant-size ciphertext) that is not possible to obtain by the conversion by [23].

In recent works $[2,5]$, it is shown that $\mathrm{PE}$ satisfying certain specific template can be converted into $\mathrm{PE}$ for its dual predicate. In particular, it yields KP-ABE-to-CP-ABE conversion. Again, their result and ours are incomparable. On the one hand, schemes obtained from their conversion are typically more efficient than ours. On the other hand, their conversion only works for schemes with the template while our conversion is completely generic. Furthermore, since they essentially exchange key and ciphertext components in the conversion, the size of keys and ciphertexts are also exchanged. For example, if we start from $\mathrm{KP}-\mathrm{ABE}$ with constant-size ciphertexts, they obtain $\mathrm{CP}-\mathrm{ABE}$ with constant-size private keys while we obtain $\mathrm{CP}-\mathrm{ABE}$ with constant-size ciphertexts.

We also remark that in the settings where PE for general circuit is available, we can easily convert any $\mathrm{KP}-\mathrm{ABE}$ into $\mathrm{CP}-\mathrm{ABE}$ by using universal circuits as discussed in $[22,20]$. However, in the settings where only PE for span programs is available, this technique is not known to be applicable. We note that all existing PE schemes for general circuits [20, 22, 9] are quite inefficient and based on strong assumptions (e.g., existence of secure multi-linear map or hardness of certain lattice problems for an exponential approximation factor). In [7], in the context of quantum computation, Belovs studies a span program that decides whether two spaces intersect or not. The problem and its solution considered there is very similar to that in Section 3 of our paper. However, he does not consider application to cryptography and the result is not applicable to our setting immediately since the syntax of span programs is slightly different.

Concurrent and Independent Work. Concurrently and independently to our work, Aggrawal and Chase [1] show specific construction of $\mathrm{CP}-\mathrm{ABE}$ scheme with constant-size ciphertexts. Compared to our CP$\mathrm{ABE}$ scheme with constant-size ciphertexts, which is obtained by our conversion, their scheme only supports monotone access structure over large universe, whereas our scheme supports non-monotonic access structure over large universe. Furthermore, we can obtain adaptively secure scheme whereas their scheme is only selectively secure. On the other hand, their construction seems to have shorter keys.

\section{Preliminaries}

Notation. Throughout the paper, $p$ denotes a prime number. We will treat a vector as a column vector, unless stated otherwise. For a vector $\mathbf{a} \in \mathbb{Z}_{p}^{n}, \mathbf{a}[i] \in \mathbb{Z}_{p}$ represents $i$-th element of the vector. Namely, $\mathbf{a}=(\mathbf{a}[1], \ldots, \mathbf{a}[n])^{\top}$. For $\mathbf{a}, \mathbf{b} \in \mathbb{Z}_{p}^{n}$, we denote their inner product as $\langle\mathbf{a}, \mathbf{b}\rangle=\mathbf{a}^{\top} \mathbf{b}=\sum_{i=1}^{n} \mathbf{a}[i] \cdot \mathbf{b}[i]$. We denote by $\mathbf{e}_{i}$ the $i$-th unit vector: its $i$-th component is one, all others are zero. $\mathbf{I}_{n}$ and $\mathbf{0}_{n \times m}$ represent an identity matrix in $\mathbb{Z}_{p}^{n \times n}$ and zero matrix in $\mathbb{Z}_{p}^{n \times m}$ respectively. We also define $\mathbf{1}_{n}=(1,1, \ldots, 1)^{\top} \in$ $\mathbb{Z}_{p}^{n}$ and $\mathbf{0}_{n}=\mathbf{0}_{n \times 1}$. We often omit the subscript if it is clear from the context. We denote by $[a, b]$ a set $\{a, a+1, \ldots, b\}$ for $a, b \in \mathbb{Z}$ such that $a \leq b$ and $[b]$ denotes $[1, b]$. For a matrix $\mathbf{X} \in \mathbb{Z}_{p}^{n \times d}$, $\operatorname{span}(\mathbf{X})$ denotes a linear space $\left\{\mathbf{X} \cdot \mathbf{u} \mid \mathbf{u} \in \mathbb{Z}_{p}^{d}\right\}$ spanned by columns of $\mathbf{X}$. For matrices $\mathbf{A} \in \mathbb{Z}_{p}^{n_{1} \times m}$ and $\mathbf{B} \in \mathbb{Z}_{p}^{n_{2} \times m},[\mathbf{A} ; \mathbf{B}] \in \mathbb{Z}_{p}^{\left(n_{1}+n_{2}\right) \times m}$ denotes $\left[\mathbf{A}^{\top}, \mathbf{B}^{\top}\right]^{\top}$ i.e., the vertical concatenation of them.

\subsection{Definition of Predicate Encryption}

Here, we define the syntax of predicate encryption. We emphasize that we do not consider attribute hiding in this paper ${ }^{3}$.

Syntax. Let $R=\left\{R_{N}: A_{N} \times B_{N} \rightarrow\{0,1\} \mid N \in \mathbb{N}^{c}\right\}$ be a relation family where $A_{N}$ and $B_{N}$ denote "ciphertext attribute" and "key attribute" spaces and $c$ is some fixed constant. The index $N=\left(n_{1}, n_{2}, \ldots, n_{c}\right)$

\footnotetext{
${ }^{3}$ This is called "public-index" predicate encryption, categorized in [11].
} 
of $R_{N}$ denotes the numbers of bounds for corresponding parameters. A predicate encryption (PE) scheme for $R$ is defined by the following algorithms:

$\operatorname{Setup}(\lambda, N) \rightarrow(\mathrm{mpk}, \mathrm{msk})$ : The setup algorithm takes as input a security parameter $\lambda$ and a index $N$ of the relation $R_{N}$ and outputs a master public key mpk and a master secret key msk.

Encrypt (mpk, $\mathrm{M}, X) \rightarrow C$ : The encryption algorithm takes as input a master public key mpk, the message $\mathrm{M}$, and a ciphertext attribute $X \in A_{N}$. It will output a ciphertext $C$.

KeyGen $(m s k, m p k, Y) \rightarrow s_{Y}$ : The key generation algorithm takes as input the master secret key msk, the master public key mpk, and a key attribute $Y \in B_{N}$. It outputs a private key sk $Y$.

$\operatorname{Decrypt}\left(\mathrm{mpk}, C, X, \mathrm{sk}_{Y}, Y\right) \rightarrow \mathrm{M}$ or $\perp$ : We assume that the decryption algorithm is deterministic. The decryption algorithm takes as input the public parameters mpk, a ciphertext $C$, ciphertext attribute $X \in A_{N}$, a private key $\mathrm{sk}_{Y}$, and private key attribute $Y$. It outputs the message $\mathrm{M}$ or $\perp$ which represents that the ciphertext is not in a valid form.

We require the standard correctness of decryption: for all $\lambda, N$, (mpk, msk) $\leftarrow \operatorname{Setup}(\lambda, N), X \in$ $A_{N}, Y \in B_{N}$ such that $R(X, Y)=1$, and sk $Y \leftarrow$ KeyGen(msk, mpk, $\left.Y\right)$, we have Decrypt(mpk, Encrypt $\left.(\mathrm{mpk}, \mathrm{M}, X), X, \mathrm{sk}_{Y}, Y\right)=\mathrm{M}$.

Security. We now define the security for an PE scheme $\Pi$ by the following game between a challenger and an attacker $\mathcal{A}$.

At first, the challenger runs the setup algorithm and gives mpk to $\mathcal{A}$. Then $\mathcal{A}$ may adaptively make key-extraction queries. We denote this phase Phase1. In this phase, if $\mathcal{A}$ submits $Y \in B_{N}$ to the challenger, the challenger returns $\mathrm{sk}_{Y} \leftarrow \operatorname{KeyGen}(\mathrm{msk}, \mathrm{mpk}, Y)$. At some point, $\mathcal{A}$ outputs two equal length messages $\mathrm{M}_{0}$ and $\mathrm{M}_{1}$ and challenge ciphertext attribute $X^{\star} \in A_{N}$. $X^{\star}$ cannot satisfy $R\left(X^{\star}, Y\right)=$ 1 for any attribute $Y$ such that $\mathcal{A}$ already queried private key for $Y$. Then the challenger flips a random coin $\beta \in\{0,1\}$, runs Encrypt $\left(\mathrm{mpk}, \mathrm{M}_{\beta}, X^{\star}\right) \rightarrow C^{\star}$ and gives challenge ciphertext $C^{\star}$ to $\mathcal{A}$. In PHASE2, $\mathcal{A}$ may adaptively make queries as in PHASE1 with following added restriction: $\mathcal{A}$ cannot make a keyextraction query for $Y$ such that $R\left(X^{\star}, Y\right)=1$. At last, $\mathcal{A}$ outputs a guess $\beta^{\prime}$ for $\beta$. We say that $\mathcal{A}$ succeeds if $\beta^{\prime}=\beta$ and denote the probability of this event by $\operatorname{Pr}_{\mathcal{A}, \Pi}^{P E}$. The advantage of an attacker $\mathcal{A}$ is defined as $A d v_{\mathcal{A}, \Pi}^{P E}=\left|\operatorname{Pr}_{\mathcal{A}, \Pi}^{P E}-\frac{1}{2}\right|$. We say that $\Pi$ is adaptively secure if $A d v_{\mathcal{A}, \Pi}^{P E}$ is negligible for all probabilistic polynomial time (PPT) adversary $\mathcal{A}$.

A weaker notion called selective security can be defined as in the above game with the exception that the adversary $\mathcal{A}$ has to choose the challenge ciphertext index $X^{\star}$ before the setup phase but private key queries $Y_{1}, \ldots, Y_{Q}$ and choice of $\left(\mathrm{M}_{0}, \mathrm{M}_{1}\right)$ can still be adaptive.

\section{2 (Arithmetic) Span Program, ABE, and Doubly Spatial Encryption}

Definition of Span Program. Let $\mathcal{U}=\left\{u_{1}, \ldots, u_{t}\right\}$ be a set of variables. For each $u_{i}$, denote $\neg u_{i}$ as a new variable. Intuitively, $u_{i}$ and $\neg u_{i}$ correspond to positive and negative attributes, respectively. Also let $\mathcal{U}^{\prime}=\left\{\neg u_{1}, \ldots, \neg u_{t}\right\}$. A span program over $\mathbb{Z}_{p}$ is specified by a pair $(\mathbf{L}, \rho)$ of a matrix and a labelling function where

$$
\mathbf{L} \in \mathbb{Z}_{p}^{\ell \times m} \quad \rho:[\ell] \rightarrow \mathcal{U} \cup \mathcal{U}^{\prime}
$$

for some integer $\ell, m$. Intuitively, the map $\rho$ labels row $i$ with attribute $\rho(i)$.

A span program accepts or rejects an input by the following criterion. For an input $\delta \in\{0,1\}^{t}$, we define the sub-matrix $\mathbf{L}_{\delta}$ of $\mathbf{L}$ to consist of the rows whose labels are set to 1 by the input $\delta$. That is, it 
consists of either rows labelled by some $u_{i}$ such that $\delta_{i}=1$ or rows labelled by some $\neg u_{i}$ such that $\delta_{i}=0$. We say that

$$
(\mathbf{L}, \rho) \text { accepts } \delta \text { iff }(1,0, \ldots, 0) \text { is in the row span of } \mathbf{L}_{\delta} \text {. }
$$

We can write this also as $\mathbf{e}_{1} \in \operatorname{span}\left(\mathbf{L}_{\delta}^{\top}\right)$. A span program is called monotone if the labels of the rows consist of only the positive literals, in $\mathcal{U}$.

Key-Policy and Ciphertext-Policy Attribute-Based Encryption. Let $\mathcal{U}$ be the universe of attributes. We define a relation $R^{\mathrm{KP}}$ on any span programs $(\mathbf{L}, \rho)$ over $\mathbb{Z}_{p}$ and any sets of attributes $S \subseteq \mathcal{U}$ as follows. For $S \subseteq \mathcal{U}$, we define $\delta \in\{0,1\}^{t}$ as an indicator vector corresponding to $S$. Namely, $\delta_{i}=1$ if $u_{i} \in S$ and $\delta_{i}=0$ if $u_{i} \notin S$. We define

$$
R^{\mathrm{KP}}(S,(\mathbf{L}, \rho))=1 \text { iff }(\mathbf{L}, \rho) \text { accepts } \delta .
$$

Similarly, $R^{\mathrm{CP}}$ is defined as $R^{\mathrm{CP}}((\mathbf{L}, \rho), S)=1$ iff $(\mathbf{L}, \rho)$ accepts $\delta$.

$\mathrm{A} \mathrm{KP}-\mathrm{ABE}$ scheme may require some bounds on parameters: we denote

$$
\begin{aligned}
\bar{k} & =\text { the maximum size of } k \text { (the size of attribute set } S \text { ), } \\
\bar{\ell} & =\text { the maximum size of } \ell \text { (the number of rows of } \mathbf{L} \text { ), } \\
\bar{m} & =\text { the maximum size of } m \text { (the number of columns of } \mathbf{L}), \\
\varphi & =\text { the maximum size of allowed repetition in }\{\rho(1), \ldots, \rho(\ell)\} .
\end{aligned}
$$

These bounds define the index $N=(\bar{k}, \bar{\ell}, \bar{m}, \varphi)$ for the predicate family. When there is no restriction on corresponding parameter, we represent it by "-" such as $(k,-,-,-)$. We define $A_{N}$ and $B_{N}$ as the set of all attribute sets and the set of all span programs whose sizes are restricted by $N$, respectively. KP-ABE is a predicate encryption for $R_{N}^{\mathrm{KP}}: A_{N} \times B_{N} \rightarrow\{0,1\}$, where $R_{N}^{\mathrm{KP}}$ is restricted on $N$ in a natural manner. $\mathrm{CP}-\mathrm{ABE}$ is defined dually with $A_{N}$ and $B_{N}$ swapped.

Let $t:=|\mathcal{U}|$. We say the scheme supports small universe if $t$ is polynomially bounded and large universe if $t$ is exponentially large. The scheme is monotonic if span programs are restricted to be monotone, and non-monotonic otherwise.

Attribute-Based Encryption for Arithmetic Span Programs [29]. In this predicate, the index $N$ for the family is specified by an integer $n$. We call it the dimension of the scheme. We define $A_{N}=\mathbb{Z}_{p}^{n}$. An arithmetic span program of dimension $n$ is specified by a tuple $(\mathbf{Y}, \mathbf{Z}, \rho)$ of two matrices $\mathbf{Y}, \mathbf{Z} \in \mathbb{Z}_{p}^{m \times \ell}$ and a map $\rho:[\ell] \rightarrow[n]$, for some integers $\ell, m$. There is no restriction on $\ell$ and $m$. If $\rho$ is restricted to injective, we say that the scheme supports only attribute one-use. Otherwise, if there is no restriction on $\rho$, we say that it is unbounded multi-use. We let $B_{N}$ be the set of all arithmetic span programs of dimension $n$. We then define

$$
R_{N}^{\mathrm{KASP}}(\mathbf{x},(\mathbf{Y}, \mathbf{Z}, \rho))=1 \text { iff } \mathbf{e}_{1} \in \operatorname{span}\left\{\mathbf{x}[\rho(j)] \cdot \mathbf{y}_{j}+\mathbf{z}_{j}\right\}_{j \in[\ell]},
$$

where here $\mathbf{e}_{1}=(1,0, \ldots, 0)^{\top} \in \mathbb{Z}_{p}^{m}$ and $\mathbf{x}[\rho(j)]$ is the $\rho(j)$-th term of $\mathbf{x}$, while $\mathbf{y}_{j}$ and $\mathbf{z}_{j}$ are the $j$ th column of $\mathbf{Y}$ and $\mathbf{Z}$ respectively. We call predicate encryption for $R^{\mathrm{KASP}}$ key-policy attribute-based encryption for arithmetic span program (KASP). Ciphertext-policy ASP (CASP) can be defined dually with $A_{N}$ and $B_{N}$ swapped.

Doubly Spatial Encryption. In this predicate, the index $N$ for the family is specified by an integer $n$ (the dimension of the scheme). We define the domains as $A_{N}=B_{N}=\mathbb{Z}_{p}^{n} \times\left(\cup_{0 \leq d \leq n} \mathbb{Z}_{p}^{n \times d}\right)$. We define

$$
R_{N}^{\mathrm{DSE}}\left(\left(\mathbf{x}_{0}, \mathbf{X}\right),\left(\mathbf{y}_{0}, \mathbf{Y}\right)\right)=1 \text { iff }\left(\mathbf{x}_{0}+\operatorname{span}(\mathbf{X})\right) \cap\left(\mathbf{y}_{0}+\operatorname{span}(\mathbf{Y})\right) \neq \emptyset .
$$


Doubly spatial encryption is PE for relation $R_{N}^{\mathrm{DSE}}$ equipped with additional key delegation algorithm defined below.

$\operatorname{KeyDel}\left(\mathrm{mpk}, \mathrm{sk}_{\left(\mathbf{y}_{0}, \mathbf{Y}\right)},\left(\mathbf{y}_{0}, \mathbf{Y}\right),\left(\hat{\mathbf{y}}_{0}, \hat{\mathbf{Y}}\right)\right) \rightarrow \mathrm{sk}_{\hat{\mathbf{Y}}}:$ The key delegation algorithm takes as input the public parameters mpk, a private key sk $\left(\mathbf{y}_{0}, \mathbf{Y}\right),\left(\mathbf{y}_{0}, \mathbf{Y}\right)$, and $\left(\hat{\mathbf{y}}_{0}, \hat{\mathbf{Y}}\right)$ such that $\hat{\mathbf{y}}_{0}+\operatorname{span}(\hat{\mathbf{Y}}) \subseteq \mathbf{y}_{0}+$ $\operatorname{span}(\mathbf{Y})$. It outputs a private key $\mathrm{sk}_{\left(\hat{\mathbf{y}}_{0}, \hat{\mathbf{Y}}\right)}$.

We require that delegation is independent of the path taken. That is, for all $\lambda, N$, (mpk, msk) $\leftarrow$ $\operatorname{Setup}(\lambda, N), \mathbf{y}_{0}, \mathbf{Y}, \hat{\mathbf{y}}_{0}, \hat{\mathbf{Y}}$ such that $\hat{\mathbf{y}}_{0}+\operatorname{span}(\hat{\mathbf{Y}}) \subseteq \mathbf{y}_{0}+\operatorname{span}(\mathbf{Y})$, we have the following distributions are identical:

$$
\operatorname{KeyDel}\left(\mathrm{mpk} \mathrm{sk}_{\left(\mathbf{y}_{0}, \mathbf{Y}\right)},\left(\mathbf{y}_{0}, \mathbf{Y}\right),\left(\hat{\mathbf{y}}_{0}, \hat{\mathbf{Y}}\right)\right) \approx \operatorname{KeyGen}\left(\mathrm{mpk}, \mathrm{msk},\left(\hat{\mathbf{y}}_{0}, \hat{\mathbf{Y}}\right)\right)
$$

where sk $\left(\mathbf{y}_{0}, \mathbf{Y}\right) \leftarrow$ KeyGen(msk, mpk, $\left.\left(\mathbf{y}_{0}, \mathbf{Y}\right)\right)$.

\subsection{Embedding Lemma for PE}

The following useful lemma from [10] describes a sufficient criterion for implication from PE for a given predicate to PE for another predicate. The lemma is applicable to any relation family. We prove the lemma in Appendix A for self-containment.

We consider two relation families:

$$
R_{N}^{\mathrm{F}}: A_{N} \times B_{N} \rightarrow\{0,1\}, \quad R_{N^{\prime}}^{\mathrm{F}^{\prime}}: A_{N^{\prime}}^{\prime} \times B_{N^{\prime}}^{\prime} \rightarrow\{0,1\},
$$

which is parametrized by $N \in \mathbb{N}^{c}$ and $N^{\prime} \in \mathbb{N}^{c^{\prime}}$ respectively. Suppose that there exists three efficient mappings

$$
f_{\mathrm{p}}: \mathbb{Z}^{c^{\prime}} \rightarrow \mathbb{Z}^{c} \quad f_{\mathrm{e}}: A_{N^{\prime}}^{\prime} \rightarrow A_{f_{\mathrm{p}}\left(N^{\prime}\right)} \quad f_{\mathrm{k}}: B_{N^{\prime}}^{\prime} \rightarrow B_{f_{\mathrm{p}}\left(N^{\prime}\right)}
$$

which maps parameters, ciphertext attributes, and key attributes, respectively, such that for all $X^{\prime} \in$ $A_{N^{\prime}}^{\prime}, Y^{\prime} \in B_{N^{\prime}}^{\prime}$

$$
R_{N^{\prime}}^{\mathrm{F}^{\prime}}\left(X^{\prime}, Y^{\prime}\right)=1 \Leftrightarrow R_{f_{\mathrm{p}}\left(N^{\prime}\right)}^{\mathrm{F}}\left(f_{\mathrm{e}}\left(X^{\prime}\right), f_{\mathrm{k}}\left(Y^{\prime}\right)\right)=1 .
$$

We can then construct a PE scheme $\Pi^{\prime}=\left\{\right.$ Setup ${ }^{\prime}$,Encrypt ${ }^{\prime}$, KeyGen ${ }^{\prime}$, Decrypt $\left.{ }^{\prime}\right\}$ for predicate $R_{N^{\prime}}^{\mathrm{F}^{\prime}}$ from a PE scheme $\Pi=\{$ Setup, Encrypt, KeyGen, Decrypt $\}$ for predicate $R_{N}^{\mathrm{F}}$ as follows. Let $\operatorname{Setup}^{\prime}\left(\lambda, N^{\prime}\right)=$ $\operatorname{Setup}\left(\lambda, f_{\mathrm{p}}\left(N^{\prime}\right)\right)$ and

$$
\begin{aligned}
\operatorname{Encrypt}^{\prime}\left(\mathrm{mpk}, \mathrm{M}, X^{\prime}\right) & =\operatorname{Encrypt}\left(\mathrm{mpk}, \mathrm{M}, f_{\mathrm{e}}\left(X^{\prime}\right)\right), \\
\operatorname{KeyGen}^{\prime}\left(\mathrm{msk}, \mathrm{mpk}, Y^{\prime}\right) & =\operatorname{KeyGen}\left(\mathrm{msk}, \mathrm{mpk}, f_{\mathrm{k}}\left(Y^{\prime}\right)\right),
\end{aligned}
$$

and $\operatorname{Decrypt}^{\prime}\left(\mathrm{mpk}, C, X^{\prime}, \mathrm{sk}_{Y^{\prime}}, Y^{\prime}\right)=\operatorname{Decrypt}\left(\mathrm{mpk}, C, f_{\mathrm{e}}\left(X^{\prime}\right), \mathrm{sk}_{Y^{\prime}}, f_{\mathrm{k}}\left(Y^{\prime}\right)\right)$.

Lemma 1 (Embedding lemma [10]). If $\Pi$ is correct and secure, then so is $\Pi^{\prime}$. This holds for selective security and adaptive security.

Intuitively, the forward and backward direction of Relation (1) ensure that the correctness and the security are preserving, respectively. 


\section{Conversion from ABE to DSE}

In this section, we show how to construct DSE for dimension $n$ from monotonic KP-ABE (with bounds on the size of attribute sets and span programs). We note that by simply swapping key and ciphertext attributes, we can also obtain CP-ABE-to-DSE conversion. We first describe the conversion, then explain the intuition behind the conversion later below.

\subsection{The Conversion}

Mapping Parameters. We map $f_{\mathrm{p}}^{\mathrm{DSE} \rightarrow \mathrm{KP}}: n \mapsto(\bar{k}, \bar{\ell}, \bar{m}, \psi)$ where

$$
\begin{aligned}
\bar{k} & =n(n+1) \kappa+1, & \bar{\ell} & =2(n \kappa+1)(n+1), \\
\bar{m} & =(n \kappa+1)(n+1)+1, & \psi & =2(n+1),
\end{aligned}
$$

where we define $\kappa:=\left\lceil\log _{2} p\right\rceil$. Moreover, we set the universe $\mathcal{U}$ as follows.

$$
\mathcal{U}=\{\operatorname{Att}[i][j][k][b] \mid(i, j, k, b) \in[0, n] \times[1, n] \times[1, \kappa] \times\{0,1\}\} \cup\{\mathrm{D}\},
$$

where $\mathrm{D}$ is a dummy attribute which will be assigned for all ciphertext. Hence, the universe size is $|\mathcal{U}|=$ $2 n(n+1) \kappa+1$. Intuitively, Att $[i][j][k][b]$ represents an indicator for the condition"the $k$-th least significant bit of the binary representation of the $j$-th element of the vector $\mathbf{x}_{i}$ is $b \in\{0,1\}$ ".

Mapping Ciphertext Attributes. For $\mathbf{x}_{0} \in \mathbb{Z}_{p}^{n}$ and $\mathbf{X}=\left[\mathbf{x}_{1}, \ldots, \mathbf{x}_{d_{1}}\right] \in \mathbb{Z}_{p}^{n \times d_{1}}$ such that $d_{1} \leq n$, we map $f_{\mathrm{e}}^{\mathrm{DSE} \rightarrow \mathrm{KP}}:\left(\mathbf{x}_{0}, \mathbf{X}\right) \mapsto S$ where

$$
S=\left\{\operatorname{Att}[i][j][k][b] \mid(i, j, k) \in\left[0, d_{1}\right] \times[1, n] \times[1, \kappa], b=\mathbf{x}_{i}[j][k]\right\} \cup\{\mathrm{D}\} .
$$

Here, we define $\mathbf{x}_{i}[j][k] \in\{0,1\}$ so that they satisfy

$$
\mathbf{x}_{i}[j]=\sum_{k=1}^{\kappa} 2^{k-1} \cdot \mathbf{x}_{i}[j][k] .
$$

That is, $\mathbf{x}_{i}[j][k]$ is the $k$-th least significant bit of the binary representation of $\mathbf{x}_{i}[j] \in \mathbb{Z}_{p}$.

Mapping Key Attributes. For $\mathbf{y}_{0} \in \mathbb{Z}_{p}^{n}$ and $\mathbf{Y}=\left[\mathbf{y}_{1}, \ldots, \mathbf{y}_{d_{2}}\right] \in \mathbb{Z}_{p}^{n \times d_{2}}$ such that $d_{2} \leq n$, we map $f_{\mathrm{k}}^{\mathrm{DSE} \rightarrow \mathrm{KP}}:\left(\mathbf{y}_{0}, \mathbf{Y}\right) \mapsto(\mathbf{L}, \rho)$ as follows. Let the numbers of rows and columns of $\mathbf{L}$ be

$$
\ell=(2 n \kappa+1)(n+1)+d_{2}+1, \quad m=(n \kappa+1)(n+1)+1,
$$

respectively. We then define

$$
\mathbf{L}=\left(\begin{array}{ccccccc}
\mathbf{e}_{1} & \mathbf{e}_{1}+\mathbf{e}_{d_{2}+2} & \mathbf{y}_{0}^{\top} & & & & \\
& & \mathbf{Y}^{\top} & & & \\
& & \mathbf{E} & \mathbf{J} & & & \\
& & \mathbf{E} & & \mathbf{J} & & \\
& & & & \ddots & \\
& & \mathbf{E} & & & \mathbf{J}
\end{array}\right) \in \mathbb{Z}_{p}^{\ell \times m}
$$


of which each sub-matrix $\mathbf{E}$ and $\mathbf{J}$ both appears $n+1$ times, where we define

$$
\mathbf{E}=\left(\begin{array}{cccc}
\mathbf{g} & & & \\
& \mathbf{g} & & \\
& & \ddots & \\
& & & \mathbf{g} \\
0 & 0 & \ldots & 0
\end{array}\right) \in \mathbb{Z}_{p}^{(2 n \kappa+1) \times n}, \quad \mathbf{J}=\left(\begin{array}{cccc}
-1 & & & \\
-1 & & & \\
& -1 & & \\
-1 & & \\
& & \ddots & \\
& & & -1 \\
& & & -1 \\
1 & 1 & \ldots & 1
\end{array}\right) \in \mathbb{Z}_{p}^{(2 n \kappa+1) \times n \kappa}
$$

where $\mathbf{g}=\left(0,1,0,2, \ldots, 0,2^{i}, \ldots, 0,2^{\kappa-1}\right)^{\top} \in \mathbb{Z}_{p}^{2 \kappa}$.

Next, we define the map $\rho:[1, \ell] \rightarrow \mathcal{U}$ as follows.

- If $i \leq d_{2}+1$, we set $\rho(i):=\mathrm{D}$.

- Else, we have $i \in\left[d_{2}+2, \ell\right]$. We then write

$$
i=\left(d_{2}+1\right)+(2 n \kappa+1) i^{\prime}+i^{\prime \prime}
$$

with a unique $i^{\prime} \in[0, n+1]$ and a unique $i^{\prime \prime} \in[0,2 n \kappa]$.

- If $i^{\prime \prime}=0$, we again set $\rho(i)=\mathrm{D}$.

- Else, we have $i^{\prime \prime} \in[1,2 n \kappa]$. We then write

$$
i^{\prime \prime}=2 \kappa j^{\prime}+2 k^{\prime}+b^{\prime}+1
$$

with unique $j^{\prime} \in[0, n-1], k^{\prime} \in[0, \kappa-1]$, and $b^{\prime} \in\{0,1\}$. We finally set

$$
\rho(i)=\operatorname{Att}\left[i^{\prime}\right]\left[j^{\prime}+1\right]\left[k^{\prime}+1\right]\left[b^{\prime}\right] .
$$

Intuition. We explain the intuition behind the conversion. $S$ can be seen as a binary representation of the information of $\left(\mathbf{x}_{0}, \mathbf{X}\right)$. In the span program $(\mathbf{L}, \rho), \mathbf{E}$ is used to reproduce the information of $\left(\mathbf{x}_{0}, \mathbf{X}\right)$ in the matrix while $\mathbf{J}$ is used to constrain the form of linear combination among rows to a certain form. ${ }^{4}$ In some sense, the roll of the lower part of the matrix $\mathbf{L}$ (the last $(2 n \kappa+1)(n+1)$ rows) is similar to universal circuit while the upper part of the matrix contains the information of $\left(\mathbf{y}_{0}, \mathbf{Y}\right)$.

\subsection{Correctness of the Conversion}

We show the following theorem. The implication from KP-ABE to DSE would then follow from the embedding lemma (Lemma 1).

Theorem 1. For $n \in \mathbb{N}$, for any $\mathbf{x}_{0} \in \mathbb{Z}_{p}^{n}, \mathbf{X} \in \mathbb{Z}_{p}^{n \times d_{1}}, \mathbf{y}_{0} \in \mathbb{Z}_{p}^{n}$ and $\mathbf{Y} \in \mathbb{Z}_{p}^{n \times d_{2}}$, it holds that

$$
R_{N}^{\mathrm{KP}}(S,(\mathbf{L}, \rho))=1 \Leftrightarrow R_{n}^{\mathrm{DSE}}\left(\left(\mathbf{x}_{0}, \mathbf{X}\right),\left(\mathbf{y}_{0}, \mathbf{Y}\right)\right)=1
$$

with $N=f_{\mathrm{p}}^{\mathrm{DSE} \rightarrow \mathrm{KP}}(n), S=f_{\mathrm{e}}^{\mathrm{DSE} \rightarrow \mathrm{KP}}\left(\mathbf{x}_{0}, \mathbf{X}\right)$, and $(\mathbf{L}, \rho)=f_{\mathrm{k}}^{\mathrm{DSE} \rightarrow \mathrm{KP}}\left(\mathbf{y}_{0}, \mathbf{Y}\right)$.

\footnotetext{
${ }^{4}$ A somewhat similar technique to ours that restricts the form of linear combination of vectors was used in [8] in a different context (for constructing a monotone span program that tests co-primality of two numbers).
} 
Proof. Define $I \subset[\ell]$ as $I:=\{i \mid \rho(i) \in S\}$ and define $\mathbf{L}_{I}$ as the sub-matrix of $\mathbf{L}$ formed by all the rows of which index is in $I$. From the definition of $f_{\mathrm{e}}^{\mathrm{DSE} \rightarrow \mathrm{KP}}$, we have that $\mathbf{L}_{I}$ is in the form of

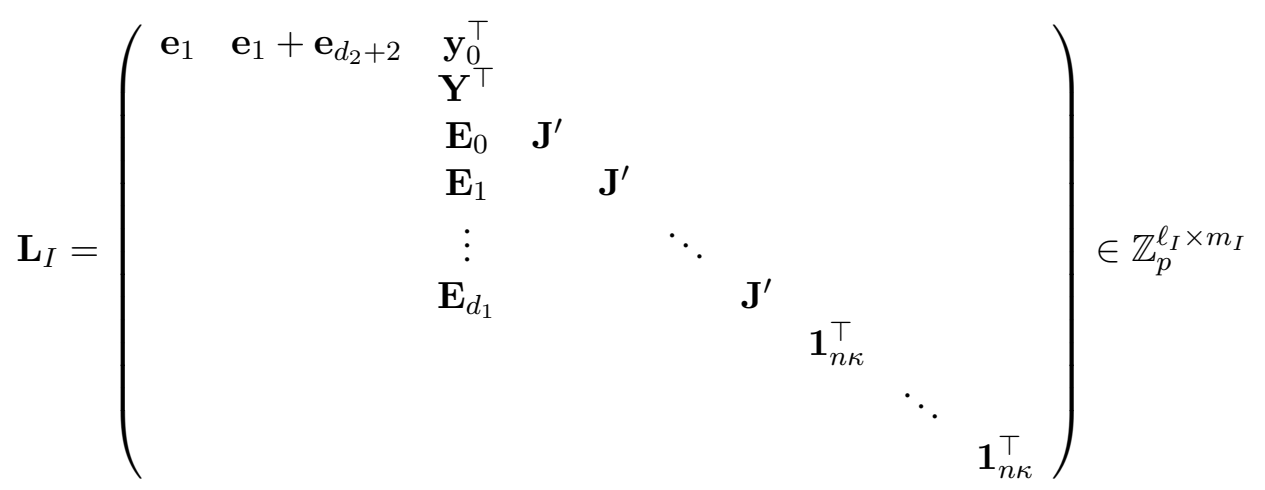

where $\ell_{I}:=(n \kappa+1)\left(d_{1}+1\right)+n-d_{1}+d_{2}+1$ and $m_{I}:=(n \kappa+1)(n+1)+1$ and

$$
\mathbf{E}_{i}=\left(\begin{array}{cccc}
\mathbf{g}_{i, 1} & & & \\
& \mathbf{g}_{i, 2} & & \\
& & \ddots & \\
& & & \mathbf{g}_{i, n} \\
0 & 0 & \ldots & 0
\end{array}\right) \in \mathbb{Z}_{p}^{(n \kappa+1) \times n}, \quad \mathbf{J}^{\prime}=\left(\begin{array}{cccc}
-1 & & & \\
& -1 & & \\
& & \ddots & \\
& & & -1 \\
1 & 1 & \ldots & 1
\end{array}\right) \in \mathbb{Z}_{p}^{(n \kappa+1) \times n \kappa} .
$$

for $i \in\left[0, d_{1}\right]$, where

$$
\mathbf{g}_{i, j}=\left(\mathbf{x}_{i}[j][1], 2 \mathbf{x}_{i}[j][2], \ldots, 2^{\kappa-1} \mathbf{x}_{i}[j][\kappa]\right)^{\top} \in \mathbb{Z}_{p}^{\kappa}
$$

We remark that it holds that $\left\langle\mathbf{1}_{\kappa}, \mathbf{g}_{i, j}\right\rangle=\mathbf{x}_{i}[j]$ by the definition of $\mathbf{x}_{i}[j][k]$ and thus $\mathbf{E}_{i}^{\top} \cdot \mathbf{1}_{n \kappa+1}=\mathbf{x}_{i}$ holds. We also remark that if $\mathbf{v}^{\top} \mathbf{J}^{\prime}=\mathbf{0}$ holds for some $\mathbf{v} \in \mathbb{Z}_{p}^{n \kappa+1}$, then there exists $v \in \mathbb{Z}_{p}$ such that $\mathbf{v}=v \mathbf{1}_{n \kappa+1}$. These properties will be used later.

To prove the theorem statement is now equivalent to prove that

$$
\mathbf{e}_{1} \in \operatorname{span}\left(\mathbf{L}_{I}^{\top}\right) \quad \Leftrightarrow \quad\left(\mathbf{x}_{0}+\operatorname{span}(\mathbf{X})\right) \cap\left(\mathbf{y}_{0}+\operatorname{span}(\mathbf{Y})\right) \neq \emptyset .
$$

Forward Direction $(\Rightarrow)$. Suppose $\mathbf{e}_{1} \in \operatorname{span}\left(\mathbf{L}_{I}^{\top}\right)$. Then, there exists $\mathbf{u} \in \mathbb{Z}_{p}^{\left((n \kappa+1)\left(d_{1}+1\right)+n-d_{1}+d_{2}+1\right)}$ such that $\mathbf{u}^{\top} \mathbf{L}_{I}=\mathbf{e}_{1}^{\top}$. We write $\mathbf{u}$ as

$$
\mathbf{u}^{\top}=(\underbrace{v}_{1}, \underbrace{\mathbf{v}^{\top}}_{d_{2}}, \underbrace{\mathbf{u}_{0}^{\top}}_{n \kappa+1}, \underbrace{\mathbf{u}_{1}^{\top}}_{n \kappa+1}, \ldots, \underbrace{\mathbf{u}_{d_{1}}^{\top}}_{n \kappa+1}, \underbrace{u_{d_{1}+1}}_{1}, \ldots, \underbrace{u_{n}}_{1}) .
$$

We then write

$$
\begin{aligned}
\mathbf{u}^{\top} \mathbf{L}_{I}=\left(v,\left(v+\left\langle\mathbf{u}_{0}, \mathbf{e}_{1}\right\rangle\right),\left(v \mathbf{y}_{0}^{\top}+\mathbf{v}^{\top} \mathbf{Y}^{\top}+\sum_{i=0}^{d_{1}} \mathbf{u}_{i}^{\top} \mathbf{E}_{i}\right),\left(\mathbf{u}_{0}^{\top} \cdot \mathbf{J}^{\prime}\right), \ldots,\right. \\
\left.\quad\left(\mathbf{u}_{d_{1}}^{\top} \cdot \mathbf{J}^{\prime}\right),\left(u_{d_{1}+1} \mathbf{1}_{n \kappa+1}^{\top}\right), \ldots,\left(u_{n} \mathbf{1}_{n \kappa+1}^{\top}\right)\right)
\end{aligned}
$$

Since $\mathbf{u}^{\top} \mathbf{L}_{I}=\mathbf{e}_{1}^{\top}$, we have $u_{d_{1}+1}=\cdots=u_{n}=0$, by comparing each element of the vector. Furthermore, since $\mathbf{u}_{i}^{\top} \cdot \mathbf{J}^{\prime}=\mathbf{0}$ for $i \in\left[0, d_{1}\right]$, there exist $\left\{u_{i} \in \mathbb{Z}_{p}\right\}_{i \in\left[0, d_{1}\right]}$ such that $\mathbf{u}_{i}=u_{i} \mathbf{1}_{n \kappa+1}$. By comparing 
the first and the second element of the vector, we obtain $v=1$ and $v+\left\langle\mathbf{u}_{0}, \mathbf{e}_{1}\right\rangle=1+u_{0}\left\langle\mathbf{1}^{\top}, \mathbf{e}_{n \kappa+1}\right\rangle=$ $1+u_{0}=0$. Hence, $u_{0}=-1$. Finally, we have that $\sum_{i=0}^{d_{1}} \mathbf{u}_{i}^{\top} \mathbf{E}_{i}+v \mathbf{y}_{0}^{\top}+\mathbf{v}^{\top} \mathbf{Y}^{\top}=\mathbf{0}$ and thus

$$
-\sum_{i=0}^{d_{1}} \mathbf{E}_{i}^{\top} \mathbf{u}_{i}=\mathbf{y}_{0}+\mathbf{Y} \cdot \mathbf{v}
$$

The left hand side of the equation is

$$
\begin{aligned}
-\sum_{i=0}^{d_{1}} \mathbf{E}_{i}^{\top} \mathbf{u}_{i} & =-u_{0} \mathbf{E}_{0}^{\top} \cdot \mathbf{1}_{n \kappa+1}-\sum_{i=1}^{d_{1}} u_{i} \mathbf{E}_{i}^{\top} \cdot \mathbf{1}_{n \kappa+1} \\
& =\mathbf{x}_{0}-\sum_{i=1}^{d_{1}} u_{i} \cdot \mathbf{x}_{i} \in\left(\mathbf{x}_{0}+\operatorname{span}(\mathbf{X})\right) .
\end{aligned}
$$

while the right hand side is $\mathbf{y}_{0}+\mathbf{Y} \cdot \mathbf{v} \in\left(\mathbf{y}_{0}+\operatorname{span}(\mathbf{Y})\right)$. This implies that $\left(\mathbf{x}_{0}+\operatorname{span}(\mathbf{X})\right) \cap\left(\mathbf{y}_{0}+\right.$ $\operatorname{span}(\mathbf{Y})) \neq \emptyset$.

Converse Direction $(\Leftarrow)$. Suppose $\left(\mathbf{x}_{0}+\operatorname{span}(\mathbf{X})\right) \cap\left(\mathbf{y}_{0}+\operatorname{span}(\mathbf{Y})\right) \neq \emptyset$. Hence, there exist sets $\left\{u_{i} \in \mathbb{Z}_{p}\right\}_{i \in\left[1, d_{1}\right]}$ and $\left\{v_{i} \in \mathbb{Z}_{p}\right\}_{i \in\left[1, d_{2}\right]}$ such that $\mathbf{x}_{0}+\sum_{i=1}^{d_{1}} u_{i} \mathbf{x}_{i}=\mathbf{y}_{0}+\sum_{i=1}^{d_{2}} v_{i} \mathbf{y}_{i}$. We set a vector $\mathbf{u}$ as

$$
\left.\mathbf{u}^{\top}=(1, \underbrace{v_{1}, \ldots, v_{d_{2}}}_{d_{2}} \underbrace{-\mathbf{1}_{n \kappa+1}^{\top},-u_{1} \mathbf{1}_{n \kappa+1}^{\top}, \ldots,-u_{d_{1}} \mathbf{1}_{n \kappa+1}^{\top}}_{(n \kappa+1)\left(d_{1}+1\right)}, \underbrace{0, \ldots, 0}_{n-d_{1}})\right) .
$$

Therefore, we have

$$
\begin{aligned}
\mathbf{u}^{\top} \mathbf{L}_{I}= & \left(1,1-1,\left(\mathbf{y}_{0}^{\top}+\sum_{i=1}^{d_{2}} v_{i} \mathbf{y}_{i}^{\top}-\mathbf{1}_{n \kappa+1}^{\top}\left(\mathbf{E}_{0}+\sum_{i=1}^{d_{1}} u_{i} \mathbf{E}_{i}\right)\right)\right. \\
& \left.\left(-\mathbf{1}_{n \kappa+1}^{\top} \mathbf{J}^{\prime}\right),\left(-u_{1} \mathbf{1}_{n \kappa+1}^{\top} \mathbf{J}^{\prime}\right), \ldots,\left(-u_{n} \mathbf{1}_{n \kappa+1}^{\top} \mathbf{J}^{\prime}\right), 0 \ldots, 0\right) \\
= & \left(1,0,\left(\mathbf{y}_{0}^{\top}+\sum_{i=1}^{d_{2}} v_{i} \mathbf{y}_{i}^{\top}\right)-\left(\mathbf{x}_{0}^{\top}+\sum_{i=1}^{d_{1}} u_{i} \mathbf{x}_{i}^{\top}\right), 0 \ldots, 0\right)=\mathbf{e}_{1}^{\top}
\end{aligned}
$$

as desired. This concludes the proof of the theorem.

\section{From DSE to Non-Monotonic ABE}

In [26], it is shown that DSE can be converted into monotonic CP-ABE with large universe (and bounds on the size of attribute sets and span programs). In this section, we extend their result to show that nonmonotonic CP-ABE with large universe and the same bounds can be constructed from DSE. We note that our transformation is very different from that of [26] even if we only consider monotonic CP-ABE because of expositional reasons. We also note that by simply swapping key and ciphertext attributes, we immediately obtain DSE-to-non-monotonic-KP-ABE conversion. Again, we first describe the conversion, provide some intuition later below. 


\subsection{The Conversion}

Mapping Parameters. We map $f_{\mathrm{p}}^{\mathrm{CP} \rightarrow \mathrm{DSE}}:(\bar{k}, \bar{\ell}, \bar{m}, \bar{\ell}) \mapsto n=4 \bar{\ell}+\bar{m}+2 \bar{k} \bar{\ell}$. We assume that the universe of attributes is $\mathbb{Z}_{p}$. This restriction can be easily removed by using collision resistant hash.

Mapping Ciphertext Attributes. For a span program $(\mathbf{L}, \rho)$, we map $f_{\mathrm{e}}^{\mathrm{CP} \rightarrow \mathrm{DSE}}:(\mathbf{L}, \rho) \mapsto\left(\mathbf{x}_{0}, \mathbf{X}\right)$ as follows. Let $\ell \times \bar{m}$ be the dimension of $\mathbf{L}$, where $\ell \leq \bar{\ell}$. (If the number of columns is smaller, we can adjust the size by padding zeroes.) Let $\ell_{0}, \ell_{1}$ be such that $\ell=\ell_{0}+\ell_{1}$, and without loss of generality, we assume that the first $\ell_{0}$ rows of $\mathbf{L}$ are associated with positive attributes and the last $\ell_{1}$ rows with negative attributes by the map $\rho$. We denote $\mathbf{L}$ as $\mathbf{L}=\left[\mathbf{L}_{0} ; \mathbf{L}_{1}\right]$ using matrices $\mathbf{L}_{0} \in \mathbb{Z}_{p}^{\ell_{0} \times \bar{m}}$ and $\mathbf{L}_{1} \in \mathbb{Z}_{p}^{\ell_{1} \times \bar{m}}$. We then define $f_{\mathrm{e}}^{\mathrm{CP} \rightarrow \mathrm{DSE}}(\mathbf{L}, \rho)=\left(\mathbf{x}_{0}, \mathbf{X}\right)$ with

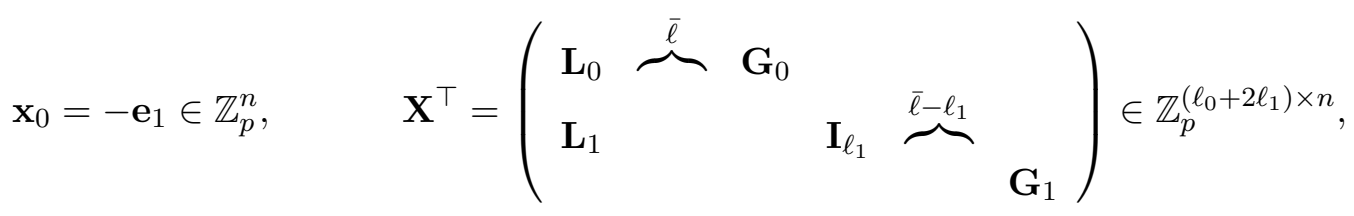

where $\mathbf{G}_{b} \in \mathbb{Z}_{p}^{\ell_{p} \times \bar{\ell}(\bar{k}+1)}$ for each $b \in\{0,1\}$ is defined as

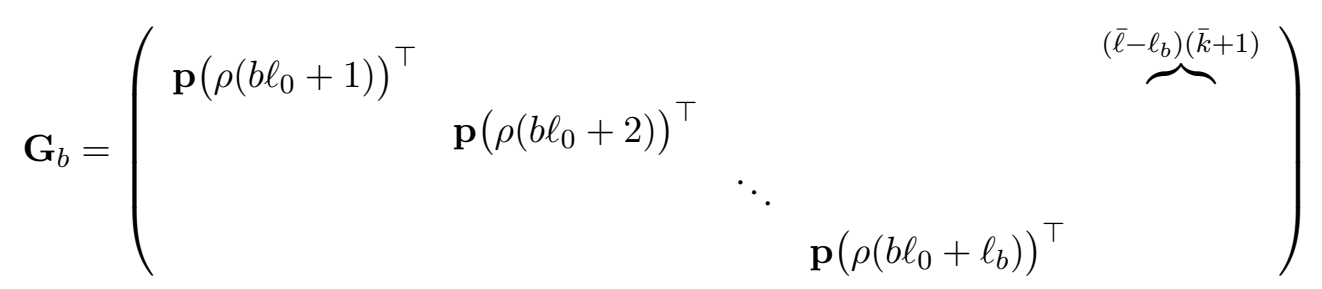

where $\mathbf{p}()$ is a function that takes an element of $\mathbb{Z}_{p}$ or its negation $\left(\left\{\neg x \mid x \in \mathbb{Z}_{p}\right\}\right)$ as an input and outputs a vector $\mathbf{p}(x)=\left(1, x, x^{2}, \ldots, x^{\bar{k}}\right)^{\top} \in \mathbb{Z}_{p}^{\bar{k}+1}$.

Mapping Key Attributes. For a set $S=\left(S_{1}, \ldots, S_{k}\right)$ such that $k \leq \bar{k}$, we map $f_{\mathrm{k}}^{\mathrm{CP} \rightarrow \mathrm{DSE}}: S \mapsto\left(\mathbf{y}_{0}, \mathbf{Y}\right)$ where

$$
\mathbf{y}_{0}=\mathbf{0}_{n} \in \mathbb{Z}_{p}^{n}, \quad \mathbf{Y}^{\top}=\left(\begin{array}{ccccc}
\overbrace{}^{\bar{m}} & \mathbf{H} & \mathbf{I}_{(\bar{k}+1) \bar{\ell}} & & \\
& & & \mathbf{H} & \mathbf{I}_{(\bar{k}+1) \bar{\ell}}
\end{array}\right) \in \mathbb{Z}_{p}^{2(\bar{k}+1) \bar{\ell} \times n},
$$

of which $\mathbf{H}$ is defined as

$$
\mathbf{H}=\mathbf{I}_{\bar{\ell}} \otimes \mathbf{q}_{S}=\left(\begin{array}{cccc}
\mathbf{q}_{S} & & & \\
& \mathbf{q}_{S} & & \\
& & \ddots & \\
& & & \mathbf{q}_{S}
\end{array}\right) \in \mathbb{Z}_{p}^{((\bar{k}+1) \bar{\ell}) \times \bar{\ell}}
$$

where $\mathbf{q}_{S}=\left(\mathbf{q}_{S}[1], \ldots, \mathbf{q}_{S}[\bar{k}+1]\right)^{\top} \in \mathbb{Z}_{p}^{\bar{k}+1}$ is defined as a coefficient vector from

$$
Q_{S}[Z]=\sum_{i=1}^{k+1} \mathbf{q}_{S}[i] \cdot Z^{i-1}=\prod_{i=1}^{k}\left(Z-S_{i}\right) .
$$

If $k<\bar{k}$, the coordinates $\mathbf{q}_{S}[k+2], \ldots, \mathbf{q}_{S}[\bar{k}+1]$ are all set to 0 .

Intuition. The matrices $\mathbf{X}$ and $\mathbf{Y}$ constructed above can be divided into two parts. The first $\ell_{0}$ rows of $\mathbf{X}^{\top}$ and the first $(\bar{k}+1) \bar{\ell}$ rows of $\mathbf{Y}^{\top}$ deal with positive attributes. The lower parts of $\mathbf{X}^{\top}$ and $\mathbf{Y}^{\top}$ deal with 
negation of attributes. Here, we explain how we handle negated attributes. Positive attributes are handled by a similar mechanism. $\mathbf{I}_{(\bar{k}+1) \bar{\ell}}$ in $\mathbf{Y}^{\top}$ and $\mathbf{G}_{1}$ in $\mathbf{X}^{\top}$ restricts the linear combination of the rows of $\mathbf{X}^{\top}$ and $\mathbf{Y}^{\top}$ to a certain form in order to two affine spaces to have a intersection. As a result, we can argue that the coefficient of the $i$-th row of $\mathbf{L}_{1}$ in the linear combination should be multiple of $Q_{S}\left(\rho\left(\ell_{0}+i\right)\right)^{5}$. Since we have that $Q_{S}(x)=0$ iff $x \in S$ for any $x \in \mathbb{Z}_{p}$, this means that the coefficient of the vector in the linear combination should be 0 if $\rho\left(\ell_{0}+i\right)=\neg$ Att and Att $\in S$. This restriction is exactly what we need to emulate predicate of non-monotonic CP-ABE.

\subsection{Correctness of the Conversion}

We show the following theorem. The implication from DSE to non-monotonic CP-ABE with large universe would then follow from the embedding lemma.

Theorem 2. For any span program $\left(\mathbf{L} \in \mathbb{Z}_{p}^{\ell \times m}, \rho\right)$ such that $\ell \leq \bar{\ell}$ and $m \leq \bar{m}$ and $S$ such that $|S| \leq \bar{k}$, let $N=(\bar{k}, \bar{\ell}, \bar{m}, \bar{\ell})$, we have that

$$
R_{n}^{\mathrm{DSE}}\left(\left(\mathbf{x}_{0}, \mathbf{X}\right),\left(\mathbf{y}_{0}, \mathbf{Y}\right)\right)=1 \Leftrightarrow R_{N}^{\mathrm{CP}}(S,(\mathbf{L}, \rho))=1
$$

where $n=f_{\mathrm{p}}^{\mathrm{CP} \rightarrow \mathrm{DSE}}(N),\left(\mathbf{x}_{0}, \mathbf{X}\right)=f_{\mathrm{e}}^{\mathrm{CP} \rightarrow \mathrm{DSE}}(\mathbf{L}, \rho)$, and $\left(\mathbf{y}_{0}, \mathbf{Y}\right)=f_{\mathrm{k}}^{\mathrm{CP} \rightarrow \mathrm{DSE}}(S)$.

Proof. Let $I \subset[1, \ell]$ be $I=\{i \mid(\rho(i)=$ Att $\wedge$ Att $\in S) \vee(\rho(i)=\neg$ Att $\wedge$ Att $\notin S)\}$. We also let $\mathbf{L}_{I}$ be the sub-matrix of $\mathbf{L}$ formed by rows whose index is in $I$.

To prove the theorem statement is equivalent to prove that

$$
\left(\mathbf{x}_{0}+\operatorname{span}(\mathbf{X})\right) \cap\left(\mathbf{y}_{0}+\operatorname{span}(\mathbf{Y})\right) \neq \emptyset \quad \Leftrightarrow \quad \mathbf{e}_{1} \in \operatorname{span}\left(\mathbf{L}_{I}^{\top}\right) .
$$

Forward Direction $(\Rightarrow)$. Suppose that there exist $\mathbf{u} \in \mathbb{Z}_{p}^{\ell_{0}+2 \ell_{1}}$ and $\mathbf{v} \in \mathbb{Z}_{p}^{2(\bar{k}+1) \bar{\ell}}$ such that $\mathbf{x}_{0}^{\top}+\mathbf{u}^{\top} \mathbf{X}^{\top}=$ $\mathbf{y}_{0}^{\top}+\mathbf{v}^{\top} \mathbf{Y}^{\top}=\mathbf{v}^{\top} \mathbf{Y}^{\top}$. We denote these vectors as

$$
\mathbf{u}^{\top}=(\underbrace{\mathbf{u}_{0}^{\top}}_{\ell_{0}}, \underbrace{\mathbf{u}_{1}^{\top}}_{\ell_{1}}, \underbrace{\mathbf{u}_{2}^{\top}}_{\ell_{1}}), \quad \mathbf{v}^{\top}=(\underbrace{\mathbf{v}_{1}^{\top}}_{\bar{k}+1}, \ldots, \underbrace{\mathbf{v}_{\bar{\ell}}^{\top}}_{\bar{k}+1} \underbrace{\mathbf{w}_{1}^{\top}}_{\bar{k}+1}, \ldots, \underbrace{\mathbf{w}_{\bar{\ell}}^{\top}}_{\bar{k}+1} .)
$$

Hence, $\mathbf{x}_{0}^{\top}+\mathbf{u}^{\top} \mathbf{X}$ and $\mathbf{v}^{\top} \mathbf{Y}$ can be written as

$$
\begin{aligned}
& \mathbf{x}_{0}^{\top}+\mathbf{u}^{\top} \mathbf{X}=(\underbrace{-\mathbf{e}_{1}^{\top}+\mathbf{u}_{0}^{\top} \mathbf{L}_{0}+\mathbf{u}_{1}^{\top} \mathbf{L}_{1}}_{\bar{m}}, \mathbf{0}_{\bar{\ell}}^{\top}, \underbrace{\mathbf{u}_{0}[1] \cdot \mathbf{p}(\rho(1))^{\top}, \ldots, \mathbf{u}_{0}\left[\ell_{0}\right] \cdot \mathbf{p}\left(\rho\left(\ell_{0}\right)\right)^{\top}}_{(\bar{k}+1) \ell_{0}}, \\
& \mathbf{0}_{\left(\bar{\ell}-\ell_{0}\right)(\bar{k}+1)}^{\top}, \underbrace{\mathbf{u}_{1}^{\top}}_{\ell_{1}}, \mathbf{0}_{\bar{\ell}-\ell_{1}}^{\top}, \\
& \underbrace{\mathbf{u}_{2}[1] \cdot \mathbf{p}\left(\rho\left(\ell_{0}+1\right)\right)^{\top}, \ldots, \mathbf{u}_{2}\left[\ell_{1}\right] \cdot \mathbf{p}\left(\rho\left(\ell_{0}+\ell_{1}\right)\right)^{\top}}_{(\bar{k}+1) \ell_{1}}, \mathbf{0}_{\left(\bar{\ell}-\ell_{1}\right)(\bar{k}+1)}^{\top})
\end{aligned}
$$

and

$$
\begin{array}{r}
\mathbf{v}^{\top} \mathbf{Y}=(\mathbf{0}_{\bar{m}}^{\top}, \underbrace{\left\langle\mathbf{v}_{1}, \mathbf{q}_{S}\right\rangle, \ldots,\left\langle\mathbf{v}_{\bar{\ell}}, \mathbf{q}_{S}\right\rangle}_{\bar{\ell}}, \underbrace{\mathbf{v}_{1}^{\top}, \ldots, \mathbf{v}_{\bar{\ell}}^{\top}}_{(\bar{k}+1) \bar{\ell}}, \\
\underbrace{\left\langle\mathbf{w}_{1}, \mathbf{q}_{S}\right\rangle, \ldots,\left\langle\mathbf{w}_{\bar{\ell}}, \mathbf{q}_{S}\right\rangle}_{\bar{\ell}}, \underbrace{\left.\mathbf{w}_{1}^{\top}, \ldots, \mathbf{w}_{\bar{\ell}}^{\top}\right)}_{(\bar{k}+1) \bar{\ell}} .
\end{array}
$$

\footnotetext{
${ }^{5}$ Here, We treat negated attributes $\left(\left\{\neg x \mid x \in \mathbb{Z}_{p}\right\}\right)$ as elements of $\mathbb{Z}_{p}$. Namely, if $\rho\left(\ell_{0}+i\right)=\neg$ Att for some Att $\in \mathbb{Z}_{p}$, $Q_{S}\left(\rho\left(\ell_{0}+i\right)\right):=Q_{S}($ Att $)$.
} 
First, by comparing the $\bar{m}+\bar{\ell}+1$-th to $\bar{m}+(\bar{k}+2) \bar{\ell}$-th elements of the vector, we obtain that $\mathbf{v}_{i}=\mathbf{u}_{0}[i] \cdot \mathbf{p}(\rho(i))$ for $i \in\left[1, \ell_{0}\right]$ and $\mathbf{v}_{i}=\mathbf{0}_{\bar{k}+1}$ for $i \in\left[\ell_{0}+1, \bar{\ell}\right]$. Furthermore, by comparing $\bar{m}+1$-th to $\bar{m}+\bar{\ell}$-th elements of the vector, we have

$$
\left\langle\mathbf{v}_{i}, \mathbf{q}_{S}\right\rangle=\mathbf{u}_{0}[i] \cdot\left\langle\mathbf{p}(\rho(i)), \mathbf{q}_{S}\right\rangle=\mathbf{u}_{0}[i] \cdot Q_{S}(\rho(i))=0
$$

for $i \in\left[1, \ell_{0}\right]$. The second equation above follows from the definition of $\mathbf{p}()$ and $\mathbf{q}_{S}$. Since $Q_{S}(\rho(i))=$ $\prod_{\omega \in S}(\rho(i)-\omega) \neq 0$ if $\rho(i) \notin S$, we have that $\mathbf{u}_{0}[i]=0$ if $\rho(i) \notin S$. That is, $\mathbf{u}_{0}[i]=0$ for $i \in\left[1, \ell_{0}\right] \backslash I$.

Next, by comparing the last $(\bar{k}+1) \bar{\ell}$ elements in the vector, we obtain that $\mathbf{w}_{i}=\mathbf{u}_{2}[i] \cdot \mathbf{p}\left(\rho\left(\ell_{0}+i\right)\right)$ for $i \in\left[1, \ell_{1}\right]$ and $\mathbf{w}_{i}=\mathbf{0}_{\bar{k}+1}$ for $i \in\left[\ell_{1}+1, \bar{\ell}\right]$. By comparing the $\bar{m}+(\bar{k}+2) \bar{\ell}+1$-th to $\bar{m}+(\bar{k}+3) \bar{\ell}$-th elements in the vector, we have that $\left(\mathbf{u}_{1}^{\top}, \mathbf{0}_{\bar{\ell}-\ell_{1}}^{\top}\right)=\left(\left\langle\mathbf{w}_{1}, \mathbf{q}_{S}\right\rangle, \ldots,\left\langle\mathbf{w}_{\bar{\ell}}, \mathbf{q}_{S}\right\rangle\right)$ and thus

$$
\mathbf{u}_{1}[i]=\left\langle\mathbf{w}_{i}, \mathbf{q}_{S}\right\rangle=\mathbf{u}_{2}[i] \cdot\left\langle\mathbf{p}\left(\rho\left(\ell_{0}+i\right)\right), \mathbf{q}_{S}\right\rangle=\mathbf{u}_{2}[i] \cdot Q_{S}\left(\rho\left(\ell_{0}+i\right)\right)
$$

holds for $i \in\left[1, \ell_{1}\right]$. From the above, we have that $\mathbf{u}_{1}[i]=0$ if $\rho\left(\ell_{0}+i\right)=\neg$ Att and Att $\in S$ for some Att. This implies that $\mathbf{u}_{1}[i]=0$ if $\left(\ell_{0}+i\right) \notin I$ for $i \in\left[1, \ell_{1}\right]$.

Finally, by comparing the first $\bar{m}$ elements in the vector, we obtain that $-\mathbf{e}_{1}^{\top}+\mathbf{u}_{0}^{\top} \mathbf{L}_{0}+\mathbf{u}_{1}^{\top} \mathbf{L}_{1}=\mathbf{0}^{\top}$. Let $\mathbf{u}_{0, I}$ be a subvector of $\mathbf{u}_{0}$ which is obtained by deleting all elements $\mathbf{u}_{0}[i]$ for $i \notin I$. Similarly, we define $\mathbf{u}_{1, I}$ as a vector obtained by deleting all elements $\mathbf{u}_{1}[i]$ for $i$ such that $\left(\ell_{0}+i\right) \notin I$ from $\mathbf{u}_{1}$. Since $\mathbf{u}_{0}[i]=0$ for $i \in\left[1, \ell_{0}\right] \backslash I$ and $\mathbf{u}_{1}[i]=0$ for $i \in\left[1, \ell_{1}\right]$ such that $\left(\ell_{0}+i\right) \notin I$, it follows that $\left(\mathbf{u}_{0, I}^{\top}, \mathbf{u}_{1, I}^{\top}\right) \mathbf{L}_{I}=\mathbf{u}_{0}^{\top} \mathbf{L}_{0}+\mathbf{u}_{1}^{\top} \mathbf{L}_{1}=\mathbf{e}_{1}^{\top}$ and thus $\mathbf{e}_{1} \in \operatorname{span}\left(\mathbf{L}_{I}^{\top}\right)$ as desired.

Converse Direction $(\Leftarrow)$. The converse direction can be shown by repeating the above discussion in reverse order. Assume that $\mathbf{e}_{1} \in \operatorname{span}\left(\mathbf{L}_{I}^{\top}\right)$. Then there exists $\mathbf{u}^{\prime} \in \mathbb{Z}_{p}^{|I|}$ such that $\mathbf{u}^{\prime \top} \mathbf{L}_{I}=\mathbf{e}_{1}^{\top}$. We extend $\mathbf{u}^{\prime}$ to define $\mathbf{u}^{\prime \prime} \in \mathbb{Z}_{p}^{\ell_{0}+\ell_{1}}$ so that $\mathbf{u}_{I}^{\prime \prime}=\mathbf{u}^{\prime}$ and $\mathbf{u}^{\prime \prime}[i]=0$ for $i \notin I$ hold. Here, $\mathbf{u}_{I}^{\prime \prime} \in \mathbb{Z}_{p}^{|I|}$ is a subvector of $\mathbf{u}^{\prime \prime}$ which is obtained by deleting all elements $\mathbf{u}^{\prime \prime}[i]$ for $i \notin I$. These conditions completely determine $\mathbf{u}^{\prime \prime}$. We denote this $\mathbf{u}^{\prime \prime}$ as $\mathbf{u}^{\prime \prime \top}=\left(\mathbf{u}_{0}^{\top}, \mathbf{u}_{1}^{\top}\right)$ using $\mathbf{u}_{0} \in \mathbb{Z}_{p}^{\ell_{0}}$ and $\mathbf{u}_{1} \in \mathbb{Z}_{p}^{\ell_{1}}$. We note that $\mathbf{u}_{0}^{\top} \mathbf{L}_{0}+\mathbf{u}_{1}^{\top} \mathbf{L}_{1}=\mathbf{e}_{1}^{\top}$ holds by the definition.

Next we define $\mathbf{v}_{i}$ for $i \in[\bar{\ell}]$ as $\mathbf{v}_{i}=\mathbf{u}_{0}[i] \cdot \mathbf{p}(\rho(i))$ if $i \in\left[\ell_{0}\right]$ and $\mathbf{v}_{i}=\mathbf{0}_{\bar{k}+1}$ if $i \in\left[\ell_{0}+1, \bar{\ell}\right]$. We claim that $\left\langle\mathbf{v}_{i}, \mathbf{q}_{S}\right\rangle=0$ holds for $i \in[\bar{\ell}]$. Here, we prove this. The case for $i \in\left[\ell_{0}+1, \bar{\ell}\right]$ is trivial. For the case of $i \in\left[1, \ell_{0}\right]$, we have

$$
\left\langle\mathbf{v}_{i}, \mathbf{q}_{S}\right\rangle=\mathbf{u}_{0}[i] \cdot\left\langle\mathbf{p}(\rho(i)), \mathbf{q}_{S}\right\rangle=\mathbf{u}_{0}[i] \cdot Q_{S}(\rho(i))=0 .
$$

The last equation above holds because we have $Q_{S}(\rho(i))=0$ if $i \in I$ and $\mathbf{u}_{0}[i]=0$ otherwise, by the definition of $\mathbf{u}_{0}[i]$.

We define $\mathbf{u}_{2}[i] \in \mathbb{Z}_{p}$ for $i \in\left[1, \ell_{1}\right]$ as $\mathbf{u}_{2}[i]=\mathbf{u}_{1}[i] / Q_{S}\left(\rho\left(\ell_{0}+i\right)\right)$ if $\mathbf{u}_{1}[i] \neq 0$ and $\mathbf{u}_{2}[i]=0$ if $\mathbf{u}_{1}[i]=0$. We have to show that $\mathbf{u}_{2}[i]$ are well defined by showing that $Q_{S}\left(\rho\left(\ell_{0}+i\right)\right) \neq 0$ if $\mathbf{u}_{1}[i] \neq 0$ (i.e., division by 0 does not occur). If $\mathbf{u}_{1}[i] \neq 0$, then $\left(\ell_{0}+i\right) \in I$ by the definition of $\mathbf{u}_{1}$. It implies that $\left(\rho\left(\ell_{0}+i\right)=\neg\right.$ Att $) \wedge($ Att $\notin S)$ for some Att $\in \mathbb{Z}_{p}$ and thus $Q_{S}\left(\rho\left(\ell_{0}+i\right)\right)=\prod_{\omega \in S}($ Att $-\omega) \neq 0$ holds as desired.

We also define $\mathbf{w}_{i}$ as $\mathbf{w}_{i}=\mathbf{u}_{2}[i] \cdot \mathbf{p}\left(\rho\left(\ell_{0}+i\right)\right)$ for $i \in\left[1, \ell_{1}\right]$ and $\mathbf{w}_{i}=\mathbf{0}_{\bar{k}+1}$ for $i \in\left[\ell_{1}+1, \bar{\ell}\right]$. Then, we have

$$
\left\langle\mathbf{w}_{i}, \mathbf{q}_{S}\right\rangle=\mathbf{u}_{2}[i] \cdot\left\langle\mathbf{p}\left(\rho\left(\ell_{0}+i\right)\right), \mathbf{q}_{S}\right\rangle=\mathbf{u}_{2}[i] \cdot Q_{S}\left(\rho\left(\ell_{0}+i\right)\right)=\mathbf{u}_{1}[i]
$$

for $i \in\left[1, \ell_{1}\right]$ and $\left\langle\mathbf{w}_{i}, \mathbf{q}_{S}\right\rangle=0$ for $i \in\left[\ell_{1}+1, \bar{\ell}\right]$.

Finally, we define $\mathbf{u}$ and $\mathbf{v}$ as $\mathbf{u}^{\top}=\left(\mathbf{u}_{0}^{\top}, \mathbf{u}_{1}^{\top}, \mathbf{u}_{2}^{\top}\right)$ and $\mathbf{v}^{\top}=\left(\mathbf{v}_{1}^{\top}, \ldots, \mathbf{v}_{\bar{\ell}}^{\top}, \mathbf{w}_{1}^{\top}, \ldots, \mathbf{w}_{\bar{\ell}}^{\top}\right)$. Then, Equation (5) and (6) hold. By the properties of $\mathbf{u}$ and $\mathbf{v}$ we investigated so far, it is straightforward to see that $\mathbf{x}_{0}^{\top}+\mathbf{u}^{\top} \mathbf{X}^{\top}=\mathbf{y}_{0}^{\top}+\mathbf{v}^{\top} \mathbf{Y}$ holds. This concludes the proof of the theorem. 


\section{From KP(CP)-ABE to KASP(CASP)}

In this section, we show that monotonic KP-ABE with small universe (without bounds on the size of span programs) can be converted into KASP. We note that we can also obtain CP-ABE-to-CASP conversion by simply swapping key and ciphertext attribute.

\subsection{The Conversion}

Mapping Parameters. We show how to construct KASP for dimension $n$ from monotonic KP-ABE for parameter $N=(n \kappa+1,-,-,-)$ and the size of attribute universe is $|\mathcal{U}|=2 n \kappa+1$. Here, $\kappa=\left\lceil\log _{2} p\right\rceil$. That is, we define $f_{\mathrm{p}}^{\mathrm{KASP} \rightarrow \mathrm{KP}}(n)=N$. We set the universe of attributes as

$$
\mathcal{U}=\{\operatorname{Att}[i][j][b] \mid(i, j, b) \in[1, n] \times[1, \kappa] \times\{0,1\}\} \cup\{\mathrm{D}\} .
$$

Intuitively, Att $[i][j][b]$ represents an indicator for the condition "the $j$-th least significant bit of the binary representation of $i$-th element of the vector $\mathbf{x}$ is $b \in\{0,1\}$ ". D is a dummy attribute which will be assigned for all ciphertexts.

Mapping Ciphertext Attributes. For $\mathbf{x} \in \mathbb{Z}_{p}^{n}$, we map $f_{\mathrm{e}}^{\mathrm{KASP} \rightarrow \mathrm{KP}}: \mathbf{x} \mapsto S$ where

$$
S=\{\operatorname{Att}[i][j][b] \mid(i, j) \in[1, n] \times[1, \kappa], b=\mathbf{x}[i][j]\} \cup\{\mathrm{D}\}
$$

where we define $\mathbf{x}[i][j] \in\{0,1\}$ in such a way that $\mathbf{x}[i]=\sum_{j=1}^{\kappa} 2^{j-1} \cdot \mathbf{x}[i][j]$. In other words, $\mathbf{x}[i][j]$ is the $j$-th least significant bit of the binary representation of $\mathbf{x}[i] \in \mathbb{Z}_{p}$.

Mapping Key Attributes. For an arithmetic span program $\left(\mathbf{Y}=\left(\mathbf{y}_{1}, \ldots, \mathbf{y}_{\ell}\right) \in \mathbb{Z}_{p}^{m \times \ell}, \mathbf{Z}=\left(\mathbf{z}_{1}, \ldots, \mathbf{z}_{\ell}\right) \in\right.$ $\left.\mathbb{Z}_{p}^{m \times \ell}, \rho\right)$ such that $\mathbf{Y}, \mathbf{Z} \in \mathbb{Z}_{p}^{m \times \ell}$, we define the map $f_{\mathrm{k}}^{\mathrm{KASP} \rightarrow \mathrm{KP}}:(\mathbf{Y}, \mathbf{Z}, \rho) \mapsto\left(\mathbf{L}, \rho^{\prime}\right)$ as follows. First, we define

$$
\mathbf{L}=\left(\begin{array}{ccccc}
\mathbf{G}_{1} & \mathbf{J} & & & \\
\mathbf{G}_{2} & & \mathbf{J} & & \\
\vdots & & \ddots & \\
\mathbf{G}_{\ell} & & & \mathbf{J}
\end{array}\right) \in \mathbb{Z}_{p}^{((2 \kappa+1) \ell) \times(\kappa \ell+m)}
$$

where the matrix $\mathbf{J} \in \mathbb{Z}_{p}^{(2 \kappa+1) \times \kappa}$ is defined as in Equation (3) (by setting $n=1$ ) while $\mathbf{G}_{i}$ is defined as

$$
\mathbf{G}_{i}=\left[\mathbf{g} \cdot \mathbf{y}_{i}^{\top} ; \mathbf{z}_{i}^{\top}\right]=\left(\mathbf{0}_{m}, \mathbf{y}_{i}, \mathbf{0}_{m}, 2 \mathbf{y}_{i}, \cdots, \mathbf{0}_{m}, 2^{\kappa-1} \mathbf{y}_{i}, \mathbf{z}_{i}\right)^{\top} \in \mathbb{Z}_{p}^{(2 \kappa+1) \times m}
$$

where $\mathbf{g}=\left(0,1,0,2, \ldots, 0,2^{i}, \ldots, 0,2^{\kappa-1}\right)^{\top} \in \mathbb{Z}_{p}^{2 \kappa}$.

Next, we define the map $\rho^{\prime}:[(2 \kappa+1) \ell] \rightarrow \mathcal{U}$ as follows.

- If $i=0 \bmod (2 \kappa+1)$, we set $\rho(i):=\mathrm{D}$.

- Else, we write

$$
i=(2 \kappa+1) i^{\prime}+2 j^{\prime}+b^{\prime}+1
$$

with unique $i^{\prime} \in[0, \ell-1], j^{\prime} \in[0, \kappa-1]$, and $b^{\prime} \in\{0,1\}$. We finally set

$$
\rho^{\prime}(i)=\operatorname{Att}\left[\rho\left(i^{\prime}+1\right)\right]\left[j^{\prime}+1\right]\left[b^{\prime}\right] \text {. }
$$

Intuition. $S$ can be seen as a binary representation of the information of $\mathbf{x}$. In the span program $\left(\mathbf{L}, \rho^{\prime}\right)$, $\mathbf{J}$ is used to constrain the form of linear combination among rows to a certain form. $\mathbf{G}_{i}$ as well as $\rho^{\prime}$, along with the above restriction, are designed so that linear combination of rows of $\mathbf{G}_{i}$ only can be a scalar multiple of the vector $\left(\mathbf{x}[\rho(i)] \mathbf{y}_{i}+\mathbf{z}_{i}\right)^{\top}$. Therefore, $\left(\mathbf{L}, \rho^{\prime}\right)$ essentially works as an arithmetic span program. 


\subsection{Correctness of the Conversion}

We show the following theorem. The implication from $\mathrm{KP}-\mathrm{ABE}$ with parameter $N=(n \kappa+1,-,-,-)$ to KASP with dimension $n$ would then follow from the embedding lemma.

Theorem 3. For any $\mathbf{x} \in \mathbb{Z}_{p}^{n}$, $\mathbf{Y} \in \mathbb{Z}_{p}^{m \times \ell}, \mathbf{Z} \in \mathbb{Z}_{p}^{m \times \ell}$, and $\rho:[\ell] \rightarrow[n]$, it holds that

$$
R_{N}^{\mathrm{KP}}\left(S,\left(\mathbf{L}, \rho^{\prime}\right)\right)=1 \Leftrightarrow R_{n}^{\mathrm{KASP}}(\mathbf{x},(\mathbf{Y}, \mathbf{Z}, \rho))=1
$$

where $N=f_{\mathrm{p}}^{\mathrm{KASP} \rightarrow \mathrm{KP}}(n), S=f_{\mathrm{e}}^{\mathrm{KASP} \rightarrow \mathrm{KP}}(\mathbf{x})$, and $\left(\mathbf{L}, \rho^{\prime}\right)=f_{\mathrm{k}}^{\mathrm{KASP} \rightarrow \mathrm{KP}}(\mathbf{Y}, \mathbf{Z}, \rho)$.

Proof. Define $I \subset[1,(2 \kappa+1) \ell]$ as $I=\left\{i \mid \rho^{\prime}(i) \in S\right\}$. We define $\mathbf{L}_{I}$ as the sub-matrix of $\mathbf{L}$ formed by rows whose index is in $I$. From the definition of $f_{\mathrm{e}}^{\mathrm{KASP} \rightarrow \mathrm{KP}}$, we have that $\mathbf{L}_{I}$ is in the form of

$$
\mathbf{L}_{I}=\left(\begin{array}{ccccc}
\mathbf{G}_{1}^{\prime} & \mathbf{J}^{\prime} & & & \\
\mathbf{G}_{2}^{\prime} & & \mathbf{J}^{\prime} & & \\
\vdots & & & \ddots & \\
\mathbf{G}_{\ell}^{\prime} & & & & \mathbf{J}^{\prime}
\end{array}\right) \in \mathbb{Z}_{p}^{((\kappa+1) \ell) \times(\kappa \ell+m)}
$$

where

$$
\mathbf{G}_{i}^{\prime}=\left[\mathbf{g}_{i} \cdot \mathbf{y}_{i}^{\top} ; \mathbf{z}_{i}^{\top}\right] \in \mathbb{Z}_{p}^{(\kappa+1) \times m}, \quad \mathbf{J}^{\prime}=\left(\begin{array}{cccc}
-1 & & \\
& -1 & \\
& & \ddots & \\
& & & -1 \\
1 & 1 & \ldots & 1
\end{array}\right) \in \mathbb{Z}_{p}^{(\kappa+1) \times \kappa}
$$

and where

$$
\mathbf{g}_{i}=\left(\mathbf{x}[\rho(i)][1], 2 \mathbf{x}[\rho(i)][2], \ldots, 2^{\kappa-1} \mathbf{x}[\rho(i)][\kappa]\right)^{\top} \in \mathbb{Z}_{p}^{\kappa}
$$

We note that we have $\left\langle\mathbf{1}_{\kappa}, \mathbf{g}_{i}\right\rangle=\mathbf{x}[\rho(i)]$ by the definition of $\mathbf{x}[\rho(i)][j]$ and thus $\mathbf{G}_{i}^{\prime \top} \cdot \mathbf{1}_{\kappa+1}=\mathbf{x}[\rho(i)] \mathbf{y}_{i}+\mathbf{z}_{i}$ holds. We also remark that if $\mathbf{v}^{\top} \mathbf{J}^{\prime}=\mathbf{0}$ holds for some $\mathbf{v} \in \mathbb{Z}_{p}^{\kappa+1}$, then there exists $v \in \mathbb{Z}_{p}$ such that $\mathbf{v}=v \mathbf{1}_{\kappa+1}$. These properties will be used later below.

To prove the theorem statement is equivalent to prove that

$$
\mathbf{e}_{1} \in \operatorname{span}\left(\mathbf{L}_{I}^{\top}\right) \quad \Leftrightarrow \quad \mathbf{e}_{1} \in \operatorname{span}\left(\left\{\mathbf{x}[\rho(i)] \mathbf{y}_{i}+\mathbf{z}_{i}\right\}_{i \in[\ell]}\right)
$$

Forward Direction $(\Rightarrow)$. We assume that $\mathbf{e}_{1} \in \operatorname{span}\left(\mathbf{L}_{I}^{\top}\right)$. From this, there exists $\mathbf{u} \in \mathbb{Z}_{p}^{(\kappa+1) \ell}$ such that $\mathbf{u}^{\top} \mathbf{L}_{I}=\mathbf{e}_{1}^{\top}$. We write this $\mathbf{u}$ as

$$
\mathbf{u}^{\top}=(\underbrace{\mathbf{u}_{1}^{\top}}_{\kappa+1}, \underbrace{\mathbf{u}_{2}^{\top}}_{\kappa+1}, \ldots, \underbrace{\mathbf{u}_{\ell}^{\top}}_{\kappa+1}) .
$$

Therefore, we have that

$$
\mathbf{e}_{1}^{\top}=\mathbf{u}^{\top} \cdot \mathbf{L}_{I}=\left(\sum_{i \in[\ell]} \mathbf{u}_{i}^{\top} \mathbf{G}_{i}^{\prime}, \mathbf{u}_{1}^{\top} \mathbf{J}^{\prime}, \ldots, \mathbf{u}_{\ell}^{\top} \mathbf{J}^{\prime}\right)
$$


Since $\mathbf{u}_{i}^{\top} \cdot \mathbf{J}^{\prime}=\mathbf{0}$ for $i \in[\ell]$, there exist $\left\{u_{i} \in \mathbb{Z}_{p}\right\}_{i \in[\ell]}$ such that $\mathbf{u}_{i}=u_{i} \mathbf{1}_{\kappa+1}$. Then, we have

$$
\mathbf{e}_{1}^{\top}=\sum_{i \in[\ell]} \mathbf{u}_{i}^{\top} \mathbf{G}_{i}^{\prime}=\sum_{i \in[\ell]} u_{i} \mathbf{1}_{\kappa+1}^{\top} \mathbf{G}_{i}^{\prime}=\sum_{i \in[\ell]} u_{i}\left(\mathbf{x}[\rho(i)] \cdot \mathbf{y}_{i}+\mathbf{z}_{i}\right)^{\top}
$$

This implies $\mathbf{e}_{1} \in \operatorname{span}\left(\left\{\mathbf{x}[\rho(i)] \mathbf{y}_{i}+\mathbf{z}_{i}\right\}_{i \in[\ell]}\right)$, as desired.

Converse Direction $(\Leftarrow)$. We assume that $\mathbf{e}_{1} \in \operatorname{span}\left(\left\{\mathbf{x}[\rho(i)] \mathbf{y}_{i}+\mathbf{z}_{i}\right\}_{i \in[\ell]}\right)$. Then, there exist $\left\{u_{i} \in\right.$ $\left.\mathbb{Z}_{p}\right\}_{i \in[\ell]}$ such that $\sum_{i \in[\ell]} u_{i}\left(\mathbf{x}[\rho(i)] \cdot \mathbf{y}_{i}+\mathbf{z}_{i}\right)=\mathbf{e}_{1}$. We set a vector $\mathbf{u} \in \mathbb{Z}_{p}^{(\kappa+1) \ell}$ as

$$
\mathbf{u}^{\top}=\left(u_{1} \mathbf{1}_{\kappa+1}^{\top}, \ldots, u_{\ell} \mathbf{1}_{\kappa+1}^{\top}\right)
$$

Then, we have that

$$
\begin{aligned}
\mathbf{u}^{\top} \cdot \mathbf{L}_{I} & =\left(\sum_{i \in[\ell]} u_{i} \mathbf{1}_{\kappa+1}^{\top} \mathbf{G}_{i}^{\prime}, u_{1} \mathbf{1}_{\kappa+1}^{\top} \mathbf{J}^{\prime}, \ldots, \mathbf{u}_{\ell} \mathbf{1}_{\kappa+1}^{\top} \mathbf{J}^{\prime}\right) \\
& =\left(\sum_{i \in[\ell]} u_{i}\left(\mathbf{x}[\rho(i)] \mathbf{y}_{i}+\mathbf{z}_{i}\right)^{\top}, \mathbf{0}_{\kappa}^{\top}, \ldots, \mathbf{0}_{\kappa}^{\top}\right)=\mathbf{e}_{1}^{\top} .
\end{aligned}
$$

This implies $\mathbf{e}_{1} \in \operatorname{span}\left(\mathbf{L}_{I}^{\top}\right)$, as desired. This concludes the proof of the theorem.

\section{Implications of Our Result}

In this section, we discuss consequences of our results.

Equivalence between (bounded) ABE and DSE. We have shown that monotonic KP/CP-ABE for $(\bar{k}, \bar{\ell}$, $\bar{m}, \varphi$ ) implies DSE (without delegation) in Section 3 and DSE implies non-monotonic KP/CP-ABE with large universe for $(\bar{k}, \bar{\ell}, \bar{m}, \varphi)$ in Section 4. Since non-monotonic KP/CP-ABE with large universe for $(\bar{k}, \bar{\ell}, \bar{m}, \varphi)$ trivially implies monotonic $\mathrm{KP} / \mathrm{CP}-\mathrm{ABE}$ with small universe for $(\bar{k}, \bar{\ell}, \bar{m}, \varphi)$, our results indicate that these PE schemes are essentially equivalent in the sense that they imply each other.

Equivalence between $\mathrm{K}(\mathrm{C}) \mathrm{ASP}$ and $\mathrm{KP}(\mathrm{CP})$-ABE. Next, we consider the case where there is no restriction on the size of span programs. In Section 5, we showed that monotonic KP-ABE for $((\bar{k}+1) \kappa,-,-,-)$ implies KASP for $(\bar{k},-,-,-)$. In Appendix C, we also show the converse direction. That is, we show that $\operatorname{KASP}$ for $(\bar{k}+1,-,-,-)$ implies non-monotonic $\operatorname{KP}-\mathrm{ABE}$ for $(\bar{k},-,-,-)$ with large universe. Since non-monotonic $\mathrm{KP}-\mathrm{ABE}$ for $(\bar{k},-,-,-)$ trivially implies monotonic $\mathrm{KP}-\mathrm{ABE}$ for $(\bar{k},-,-,-)$, our results indicate that these PE schemes are essentially equivalent similarly to the above case. Similar implications hold for CP-ABE. See figure 1 for the overview.

By applying the conversions to existing schemes, we obtain various new schemes. The overviews of properties of resulting schemes and comparison with existing schemes are provided in Table 1, 2, 3, and 4. All schemes in the tables are constructed in pairing groups. In the tables, we count the number of group elements to measure the size of master public keys $(|\mathrm{mpk}|)$, ciphertexts $(|C|)$, and private keys $(\mid$ sk $\mid)$. Note that our conversions only can be applied to ABE schemes supporting span programs over $\mathbb{Z}_{p}$. Therefore, for $\mathrm{ABE}$ schemes constructed on composite order groups [31,2], our conversions are not applicable since they support span programs over $\mathbb{Z}_{N}$ where $N$ is a product of several large primes. Similar restrictions are posed on DSE and K(C)ASP. Though it is quite plausible that our conversions work even in such cases assuming hardness of factoring $N$, we do not prove this in this paper. 
Table 1: Comparison among DSE Schemes

\begin{tabular}{lcccccc}
\hline Schemes & $|\mathrm{mpk}|$ & $|C|$ & $|\mathrm{sk}|$ & Delegation & Security & Assumption \\
\hline \hline Hamburg11 [26] & $O(n)$ & $O\left(d_{1}\right)$ & $O\left(d_{2}\right)$ & $\checkmark$ & Selective & Parameterized \\
CW14 [17] & $O\left(n^{2}\right)$ & $O\left(n d_{1}\right)$ & $O(n)$ & $\checkmark$ & Selective & Static \\
CZF12 [14] & $O(n)$ & $O\left(d_{1}\right)$ & $O\left(d_{2}\right)$ & $\checkmark$ & Adaptive & Static \\
\hline Sec. 3 + RW13 [39] & $O(1)$ & $O\left(n d_{1} \kappa\right)$ & $O\left(n^{2} \kappa\right)$ & $\checkmark$ & Selective & Parameterized \\
Sec. 3 + ALP11 [4] & $O\left(n^{2} \kappa\right)$ & $O(1)$ & $O\left(n^{4} \kappa^{2}\right)$ & $\checkmark$ & Selective & Parameterized \\
Sec. 3 + OT12 [37] & $O(1)$ & $O\left(n^{2} d_{1} \kappa\right)$ & $O\left(n^{2} \kappa\right)$ & $?$ & Adaptive & Static \\
Sec. 3 + A15 [3] & $O(1)$ & $O\left(n d_{1} \kappa\right)$ & $O\left(n^{2} \kappa\right)$ & $?$ & Adaptive & Parameterized \\
Sec. 3 + A15 [3] & $O\left(n^{2} \kappa\right)$ & $O(1)$ & $O\left(n^{4} \kappa^{2}\right)$ & $?$ & Adaptive & Parameterized \\
Sec. 3 + A15 [3] & $O\left(n^{2} \kappa\right)$ & $O\left(n^{4} \kappa^{2}\right)$ & $O(1)$ & $?$ & Adaptive & Parameterized \\
\hline
\end{tabular}

${ }^{\dagger} n$ is the dimension of the scheme; $d_{1}$ and $d_{2}$ denote the dimension of the space associated with the ciphertext and private key, respectively; $\kappa=\left\lceil\log _{2} p\right\rceil$.

$\ddagger$ "Delegation" shows if key delegation is supported. "?" means unknown.

New DSE Schemes. By applying our KP(CP)-ABE-to-DSE conversion to existing KP(CP)-ABE schemes, we obtain many new DSE schemes. Table 1 shows overview of obtained schemes. ${ }^{6}$ Specifically,

- From the unbounded KP-ABE schemes [37, 39, 3], we obtain the first DSE scheme with constant-size master public key (without delegation). Note that all previous schemes [26, 14, 17] require at least $O(n)$ group elements in master public key where $n$ is the dimension of the scheme.

- From KP-ABE scheme with constant-size ciphertexts [4, 28, 41, 3], we obtain the first DSE scheme with constant-size ciphertexts. All previous schemes [26,14,17] require at least $O\left(d_{1}\right)$ group elements in ciphertexts where $d_{1}$ is the dimension of the affine space associated to a ciphertext.

- From CP-ABE scheme with constant-size keys [5], we obtain the first DSE scheme with constant-size private keys. All previous schemes require at least $O\left(d_{2}\right)$ group elements in private keys where $d_{2}$ is the dimension of the affine space associated to a private key.

The schemes obtained from [37, 3] achieves adaptive security. Furthermore, for schemes obtained from $[39,4,28]$, we can define key delegation algorithm. The details of the key delegation algorithm will be given in Appendix B.

CP-ABE with Constant-Size Ciphertexts. By applying our DSE-to-non-monotonic-CP-ABE conversion in Section 4 to the DSE scheme with constant-size ciphertexts obtained above, we obtain the first non-monotonic CP-ABE with constant-size ciphertexts. Previous CP-ABE schemes with constant-size ciphertexts $[19,13,12]$ only support threshold or more limited predicates ${ }^{7}$. See Table 2 for comparison (we list only relevant schemes).

KP-ABE with Constant-Size Keys. By applying our DSE-to-non-monotonic-KP-ABE conversion in Section 4 to the DSE scheme with constant-size keys obtained above, we obtain the first non-monotonic KP-ABE with constant-size keys. See Table 3 for comparison (we list only relevant schemes).

New KASP and CASP Schemes. By applying the KP(CP)-ABE-to-K(C)ASP conversion in Section 5, we obtain many new K(C)ASP schemes. See Table 4 for the overview. Specifically,

\footnotetext{
${ }^{6}$ In the table, parameterized assumptions refer to $q$-type assumptions, which are non-interactive and falsifiable but parameterized by some parameters of the scheme such as $k, \bar{k}$.

${ }^{7}$ One would be able to obtain CP-ABE with constant-size ciphertexts supporting threshold formulae by applying the generic conversion in [23] to a KP-ABE scheme proposed in [4]. However, the resulting scheme supports more limited predicate compared to ours. To the best of our knowledge, this observation has not appeared elsewhere.
} 
Table 2: Comparison among CP-ABE Schemes

\begin{tabular}{|c|c|c|c|c|c|c|c|}
\hline \multirow[t]{2}{*}{ Schemes } & \multicolumn{2}{|c|}{ Expressiveness } & \multicolumn{3}{|c|}{ Efficiency } & \multirow[t]{2}{*}{ Security } & \multirow[t]{2}{*}{ Assumption } \\
\hline & Universe & Policy & |mpk| & $|C|$ & $|\mathrm{sk}|$ & & \\
\hline OT12 [37] & Large & Non-mono. Span. & $O(1)$ & $O(\ell)$ & $O(k \varphi)$ & Adaptive & Static \\
\hline AY15 [5], A15 [3] & Large & Mono. Span. & $O(1)$ & $O(\ell)$ & $O(k)$ & Adaptive & Parametrized \\
\hline AY15 [5], A15 [3] & Large & Mono. Span. & $O(\bar{k})$ & $O(\bar{k} \ell)$ & $O(1)$ & Adaptive & Parametrized \\
\hline $\mathrm{EMN}+09$ [19] & Small & AND-only & $O(\bar{k})$ & $O(1)$ & $O(\bar{k})$ & Selective & Static \\
\hline CZF11 [13] & Small & AND-only & $O(\bar{k})$ & $O(1)$ & $O\left(\bar{k}^{2}\right)$ & Selective & Static \\
\hline CCL+13 [12] & Small & Threshold & $O(\bar{k})$ & $O(1)$ & $O\left(\bar{k}^{2}\right)$ & Adaptive & Static \\
\hline Sec. 3,4 + ALP11 [4] & Large & Non-mono. Span. & $O\left((\bar{k} \bar{\ell})^{2} \kappa\right)$ & $O(1)$ & $O\left((\bar{k} \bar{\ell})^{4} \kappa^{2}\right)$ & Selective & Parametrized \\
\hline Sec. $3,4+\mathrm{T} 14[41]$ & Large & Non-mono. Span. & $O\left((\bar{k} \bar{\ell})^{2} \kappa\right)$ & $O(1)$ & $O\left((\bar{k} \bar{\ell})^{4} \kappa^{2}\right)$ & Semi-adapt & Static \\
\hline Sec. $3,4+$ A15 [3] & Large & Non-mono. Span. & $O\left((\bar{k} \bar{\ell})^{2} \kappa\right)$ & $O(1)$ & $O\left((\bar{k} \bar{\ell})^{4} \kappa^{2}\right)$ & Adaptive & Parametrized \\
\hline Sec. 5,C + A15 [3] & Large & Non-mono. Span. & $O(1)$ & $O(\bar{k} \kappa \ell)$ & $O(\bar{k} \kappa)$ & Adaptive & Parameterized \\
\hline Sec. 5,C + A15 [3] & Large & Non-mono. Span. & $O(\bar{k} \kappa)$ & $O\left((\bar{k} \kappa)^{2} \ell\right)$ & $O(1)$ & Adaptive & Parameterized \\
\hline
\end{tabular}

${ }^{\dagger} k$ is the size of an attribute set associated with a key, $\ell$ is the number of rows of a span program matrix associated with a ciphertext; $\bar{k}, \bar{\ell}$ are the maximums of $k, \ell$ (if bounded); $\varphi$ is the maximum number of allowed attribute multi-use in one policy (if bounded); $\kappa=\left\lceil\log _{2} p\right\rceil$.

Table 3: Comparison among KP-ABE Schemes

\begin{tabular}{|c|c|c|c|c|c|c|c|}
\hline \multirow{2}{*}{ Schemes } & \multicolumn{2}{|c|}{ Expressiveness } & \multicolumn{3}{|c|}{ Efficiency } & \multirow{2}{*}{ Security } & \multirow{2}{*}{ Assumption } \\
\hline & Universe & Policy & |mpk| & $|C|$ & $|\mathrm{sk}|$ & & \\
\hline OT12 [37] & Large & Non-mono. Span. & $O(1)$ & $O(k \varphi)$ & $O(\ell)$ & Ada & Static \\
\hline AY15 [5], A15 [3] & Large & Mono. Span. & $O(1)$ & $O(k)$ & $O(\ell)$ & Adaptive & Parameterized \\
\hline AY15 [5], A15 [3] & Large & Mono. Span. & $O(\bar{k})$ & $O(1)$ & $O(\bar{k} \ell)$ & Adaptive & Parameterized \\
\hline Sec. 3,4 + A15 [3] & Large & Non-mono. Span. & $O\left((\bar{k} \bar{\ell})^{2} \kappa\right)$ & $O\left((\bar{k} \bar{\ell})^{4} \kappa^{2}\right)$ & $O(1)$ & Adaptive & Parameterized \\
\hline Sec. $5, C+A 15[3]$ & Large & Non-mono. Span. & $O(1)$ & $O(\bar{k} \kappa)$ & $O(\bar{k} \kappa \ell)$ & Adaptive & Parameterized \\
\hline Sec. 5,C + A15 [3] & Large & Non-mono. Span. & $O(\bar{k} \kappa)$ & $O(1)$ & $O\left((\bar{k} \kappa)^{2} \ell\right)$ & Adaptive & Parameterized \\
\hline
\end{tabular}

${ }^{\dagger} k$ is the size of an attribute set associated with a ciphertext, $\ell$ is the number of rows of a span program matrix associated with a key; $\bar{k}, \bar{\ell}$ are the maximums of $k, \ell$ (if bounded); $\varphi$ is the maximum number of allowed attribute multi-use in one policy (if bounded); $\kappa=\left\lceil\log _{2} p\right\rceil$.

- From the unbounded KP-ABE, CP-ABE schemes of [39, 3], we obtain the first KASP, CASP schemes with constant-size master public key.

- From adaptively secure KP-ABE, CP-ABE schemes of [34, 3], we obtain the first adaptively secure KASP, CASP schemes with unbounded attribute multi-use.

- From KP-ABE schemes with constant-size ciphertexts [4, 28, 41, 3], we obtain the first KASP schemes with constant-size ciphertexts.

- From CP-ABE schemes with constant-size keys [3], we obtain the first CASP schemes with constantsize keys.

Until recently, the only (K)ASP scheme in the literature was proposed by [29], which is selectively secure and the master public key and ciphertext size are linear in the dimension of the scheme. Very recently, adaptively secure KASP and CASP were given in [16], albeit with the restriction of one-time use (of the same attribute in one policy).

We remark that the conversion is not applicable for schemes in [36, 37] since these schemes are KP$\mathrm{ABE}$ for $(*, *, *, \varphi)$ where $\varphi$ is polynomially bounded, whereas our conversion requires the last parameter to be unbounded. 
Table 4: Comparison among KASP and CASP Schemes

\begin{tabular}{lccccccc}
\hline Schemes & Type & $|\mathrm{mpk}|$ & $|C|$ & $|\mathrm{sk}|$ & Security & Attrib. Multi-use & Assumption \\
\hline \hline IW14 [29] & KASP & $O(n)$ & $O(n)$ & $O(\ell)$ & Selective & yes & Static \\
CGW15 [16] & KASP & $O(n)$ & $O(n)$ & $O(\ell)$ & Adaptive & no & Static \\
CGW15 [16] & CASP & $O(n)$ & $O(\ell)$ & $O(n)$ & Adaptive & no & Static \\
\hline Sec. 5 + LW12[34] & KASP & $O(n \kappa)$ & $O(n \kappa)$ & $O(\ell \kappa)$ & Adaptive & yes & Parameterized \\
Sec. 5 + ALP11[4] & KASP & $O(n \kappa)$ & $O(1)$ & $O\left(\ell n \kappa^{2}\right)$ & Selective & yes & Parameterized \\
Sec. 5 + RW13[39] & KASP & $O(1)$ & $O(n \kappa)$ & $O(\ell \kappa)$ & Selective & yes & Parameterized \\
Sec. 5 + A15[3] & KASP & $O(n \kappa)$ & $O(1)$ & $O\left(\ell n \kappa^{2}\right)$ & Adaptive & yes & Parameterized \\
Sec. 5 + A15[3] & KASP & $O(1)$ & $O(n \kappa)$ & $O(\ell \kappa)$ & Adaptive & yes & Parameterized \\
Sec. 5 + LW12[34] & CASP & $O(n \kappa)$ & $O(\ell \kappa)$ & $O(n \kappa)$ & Adaptive & yes & Parameterized \\
Sec. 5 + RW13[39] & CASP & $O(1)$ & $O(\ell \kappa)$ & $O(n \kappa)$ & Selective & yes & Parameterized \\
Sec. 5 + A15[3] & CASP & $O(1)$ & $O(\ell \kappa)$ & $O(n \kappa)$ & Adaptive & yes & Parameterized \\
Sec. 5 + A15[3] & CASP & $O(n \kappa)$ & $O\left(\ell n \kappa^{2}\right)$ & $O(1)$ & Adaptive & yes & Parameterized \\
\hline
\end{tabular}

${ }^{\dagger} n$ is the dimension of the scheme; $\ell$ is the number of the columns of the matrices that define an arithmetic span program $\left(\ell\right.$ reflects the size of an arithmetic span program); $\kappa=\left\lceil\log _{2} p\right\rceil$.

\section{Application to Attribute-Based Signature}

Here, we discuss that our techniques developed in previous sections are also applicable to construct attribute-based signatures (ABS) [35, 36]. ABS is an advanced form of signature and can be considered as a signature analogue of ABE. In particular, it resembles $\mathrm{CP}-\mathrm{ABE}$ in the sense that a private key is associated with a set of attributes while a signature is associated with a policy and a message. A user can sign on a message with a policy if and only if she has a private key associated with a set satisfying the policy. Roughly speaking, this property corresponds to the correctness and unforgeability. For ABS, we also require privacy. That is, we require that one cannot obtain any information about the attribute of the signer from a signature.

The construction of expressive ABS scheme with constant-size signatures has been open. All previous ABS schemes with constant-size signatures [27, 12] only supports threshold predicates. The difficulty of constructing ABS with constant-size signatures seems to be related to the difficulty of construction of $\mathrm{CP}-\mathrm{ABE}$ with constant-size ciphertexts. That is, it is hard to set constant number of group elements so that they include very complex information such as span programs.

To solve the problem, we first define the notion of predicate signature (PS) that is a signature analogue of PE. Then we construct a PS scheme that is dual of ABS: a private key is associated with a policy and a signature with a set. The scheme achieves constant-size signatures. This is not difficult to achieve because the signature is associated with a set which is a simpler object compared to a policy. The scheme is based on PS scheme for threshold predicate with constant-size signatures by [27]. We change the scheme mainly in two ways. At first, instead of using Shamir's secret sharing scheme, we use linear secret sharing scheme so that they support more general predicate. We also add some modification so that the signature size be even shorter. The signatures of the resulting scheme only consist of two group elements.

Since signature analogue of Lemma 1 holds (Lemma 2 in Appendix D), we can apply KP-ABE-tonon-monotonic-CP-ABE conversion (combination of the results in Section 3 and 4) to obtain the first ABS scheme with constant-size signatures supporting non-monotonic span program. See Appendix D for the details.

Acknowledgement. We would like to thank anonymous reviewers and members of Shin-Akarui-AngouBenkyou-Kai for their helpful comments. 


\section{References}

[1] S. Agrawal and M. Chase. A study of pair encodings: predicate encryption in prime order groups. IACR Cryptology ePrint Archive, 2015:413, 2015.

[2] N. Attrapadung. Dual system encryption via doubly selective security: framework, fully secure functional encryption for regular languages, and More. In EUROCRYPT, pages 557-577, 2014.

[3] N. Attrapadung. Dual System Encryption Framework in Prime-Order Groups. IACR Cryptology ePrint Archive, 2015:390, 2015.

[4] N. Attrapadung, B. Libert, and E. Panafieu. Expressive key-policy attribute-based encryption with constant-size ciphertexts. In Public Key Cryptography, pages 90-108, 2011.

[5] N. Attrapadung and S. Yamada. Duality in ABE: Converting Attribute Based Encryption for Dual Predicate and Dual Policy via Computational Encodings. In CT-RSA, pages 87-105, 2015.

[6] A. Beimel. Secure Schemes for Secret Sharing and Key Distribution. PhD thesis, Israel Institute of Technology, Technion, Haifa, Israel, 1986.

[7] A. Belovs. Span-program-based quantum algorithm for the rank problem. Technical Report arXiv:1103.0842, arXiv.org, 2011. Available from http://arxiv.org/abs/1103.0842.

[8] A. Beimel, and Y. Ishai. On the Power of Nonlinear Secret-Sharing. IEEE Conference on Computational Complexity, pages 188-202, 2001.

[9] D. Boneh, C. Gentry, S. Gorbunov, S. Halevi, V. Nikolaenko,G. Segev, V. Vaikuntanathan, and D. Vinayagamurthy. Fully key-homomorphic encryption, arithmetic circuit $\mathrm{ABE}$ and compact garbled circuits. In EUROCRYPT, pages 533-556, 2014.

[10] D. Boneh and M. Hamburg. Generalized identity based and broadcast encryption schemes. In ASIACRYPT, pages 455-470, 2008.

[11] D. Boneh, A. Sahai, and B. Waters. Functional encryption: Definitions and challenges. In TCC, pages 253-273, 2011.

[12] C. Chen, J. Chen, H. Lim, Z. Zhang, D. Feng, S. Ling, and H. Wang. Fully secure attribute-based systems with short ciphertexts/signatures and threshold access structures. In CT-RSA, pages 50-67, 2013.

[13] C. Chen, Z. Zhang, and D. Feng. Efficient ciphertext policy attribute-based encryption with constant-size ciphertext and constant computation-cost. In ProvSec, pages 84-101, 2011.

[14] C. Chen, Z. Zhang, and D. Feng. Fully secure doubly-spatial encryption under simple assumptions. In ProvSec, pages 253-263, 2012.

[15] J. Chen, H. Lim, S. Ling, and H. Wang. The relation and transformation between hierarchical inner product encryption and spatial encryption. Des. Codes Cryptography, 71(2):pages 347-364, 2014.

[16] J. Chen, R. Gay, and H. Wee. Improved Dual System ABE in Prime-Order Groups via Predicate Encodings. In Eurocrypt, 2015.

[17] J. Chen and H. Wee. Doubly spatial encryption from DBDH. Theor. Comput. Sci, 543:pages 79-89, 2014

[18] J. Chen and H. Wee. Semi-adaptive attribute-based encryption and improved delegation for Boolean formula. SCN, pages 277-297, 2014.

[19] K. Emura, A.o Miyaji, A. Nomura, K. Omote, and M. Soshi. A ciphertext-policy attribute-based encryption scheme with constant ciphertext length. In ISPEC, pages 13-23, 2009.

[20] S. Garg, C. Gentry, S. Halevi, A. Sahai, and B. Waters. Attribute-based encryption for circuits from multilinear maps. In CRYPTO (2), pages 479-499, 2013.

[21] A. Ge, R. Zhang, C. Chen, C. Ma, and Z. Zhang. Threshold ciphertext policy attribute-based encryption with constant size ciphertexts. In ACISP, pages 336-349, 2012.

[22] S. Gorbunov, V. Vaikuntanathan, and H. Wee. Attribute-based encryption for circuits. In STOC, pages 545-554, 2013.

[23] V. Goyal, A. Jain, O. Pandey, and A. Sahai. Bounded ciphertext policy attribute based encryption. In ICALP (2), pages 579-591, 2008. 
[24] V. Goyal, V. Kumar, S. V. Lokam, and M. Mahmoody. On Black-Box Reductions between Predicate Encryption Schemes. In TCC, pages 440-457, 2012.

[25] V. Goyal, O. Pandey, A. Sahai, and B. Waters. Attribute-based encryption for fine-grained access control of encrypted data. In ACM Conference on Computer and Communications Security, pages 89-98, 2006.

[26] M. Hamburg. Spatial encryption. IACR Cryptology ePrint Archive, 2011:389, 2011.

[27] J. Herranz, F. Laguillaumie, B. Libert, and C. Ràfols. Short attribute-based signatures for threshold predicates. In CT-RSA, pages 51-67, 2012.

[28] S. Hohenberger and B. Waters. Attribute-based encryption with fast decryption. In Public Key Cryptography, pages 162-179, 2013.

[29] Y. Ishai and H. Wee. Partial Garbling and Their Applications. In ICALP (1), pages 650-662, 2014.

[30] J. Katz, A. Sahai, and B. Waters. Predicate Encryption Supporting Disjunctions, Polynomial Equations, and Inner Products. In EUROCRYPT, pages 146-162, 2008.

[31] A. B. Lewko, T. Okamoto, A. Sahai, K. Takashima, and B. Waters. Fully secure functional encryption: Attribute-based encryption and (hierarchical) inner product encryption. In EUROCRYPT, pages 62-91, 2010.

[32] A. B. Lewko and B. Waters. Unbounded hibe and attribute-based encryption. In EUROCRYPT, pages 547-567, 2011.

[33] A. B. Lewko and B. Waters. Decentralizing Attribute-Based Encryption. In EUROCRYPT, pages 568-588, 2011.

[34] A. B. Lewko and B. Waters. New Proof Methods for Attribute-Based Encryption: Achieving Full Security through Selective Techniques. In CRYPTO, pages 180-198, 2012.

[35] H. K. Maji, M. Prabhakaran, and M. Rosulek. Attribute-Based Signatures. In CT-RSA, pages 376-392, 2011.

[36] Tatsuaki Okamoto and Katsuyuki Takashima. Efficient attribute-based signatures for non-monotone predicates in the standard model In PKC, pages 35-52, 2011.

[37] T. Okamoto and K. Takashima. Fully secure unbounded inner-product and attribute-based encryption. In ASIACRYPT, pages 349-366, 2012.

[38] B. Parno, M. Raykova, and V. Vaikuntanathan. How to Delegate and Verify in Public: Verifiable Computation from Attribute-Based Encryption. In TCC, pages 422-439, 2012.

[39] Y. Rouselakis and B. Waters. Practical constructions and new proof methods for large universe attribute-based encryption. In ACM Conference on Computer and Communications Security, pages 463-474, 2013.

[40] Amit Sahai and Brent Waters. Fuzzy identity-based encryption. In EUROCRYPT, pages 457-473, 2005.

[41] K. Takashima. Expressive attribute-based encryption with constant-size ciphertexts from the decisional linear assumption. SCN, pages 298-317, 2014.

[42] B. Waters. Efficient identity-based encryption without random oracles. In EUROCRYPT, pages 114-127, 2005.

[43] B. Waters. Ciphertext-Policy Attribute-Based Encryption: An Expressive, Efficient, and Provably Secure Realization. In $P K C$, pages 53-70, 2011.

[44] B. Waters. Functional Encryption for Regular Languages. In CRYPTO, pages 218-235, 2012.

[45] S. Yamada, N. Attrapadung, G. Hanaoka, and N. Kunihiro. A framework and compact constructions for nonmonotonic attribute-based encryption. In Public Key Cryptography, pages 275-292, 2014.

\section{A Proof of Lemma 1}

Proof. We prove the lemma for the case of adaptive security. The same proof works for other cases. We construct an adversary $\mathcal{B}$ against $\Pi$ from an adversary $\mathcal{A}$ against $\Pi^{\prime}$ so that $\mathcal{B}$ has the same advantage as $\mathcal{A}$ and the running time of $\mathcal{B}$ is only polynomially larger than $\mathcal{A}$. At first, $\operatorname{Setup}\left(\lambda, f_{\mathrm{p}}\left(N^{\prime}\right)\right)=$ $\operatorname{Setup}^{\prime}\left(\lambda, N^{\prime}\right) \rightarrow(\mathrm{mpk}, \mathrm{msk})$ is run and $\mathrm{mpk}$ is given to $\mathcal{B}$. $\mathcal{B}$ gives mpk to $\mathcal{A}$. In PHASE1, when $\mathcal{A}$ makes key query for $Y^{\prime} \in B_{N^{\prime}}^{\prime}, \mathcal{B}$ computes $Y=f_{\mathrm{k}}\left(Y^{\prime}\right)$ and makes a key-extraction query for its challenger. Then, KeyGen $\left(\mathrm{msk}, \mathrm{mpk}, f_{\mathrm{k}}\left(Y^{\prime}\right)\right)=\mathrm{KeyGen}^{\prime}\left(\mathrm{msk}, \mathrm{mpk}, Y^{\prime}\right) \rightarrow \mathrm{sk}_{Y^{\prime}}$ is run and $\mathcal{B}$ is given $\mathrm{sk}_{Y^{\prime}}$. Then, $\mathcal{B}$ 
gives it to $\mathcal{A}$. At some point, $\mathcal{A}$ outputs two equal length messages $\mathrm{M}_{0}$ and $\mathrm{M}_{1}$ and challenge ciphertext attribute $X^{\prime \star} \in A_{N^{\prime}}^{\prime}$. Then $\mathcal{B}$ computes $X^{\star}=f_{\mathrm{e}}\left(X^{\prime \star}\right)$ and submits $X^{\star}, \mathrm{M}_{0}$, and $\mathrm{M}_{1}$ to its challenger. Then, $\operatorname{Encrypt}\left(\mathrm{mpk}, \mathrm{M}_{\beta}, f_{\mathrm{e}}\left(X^{\prime \star}\right)\right)=\operatorname{Encrypt}^{\prime}\left(\mathrm{mpk}, \mathrm{M}_{\beta}, X^{\prime \star}\right) \rightarrow C^{\star}$ is run and $C^{\star}$ is given to $\mathcal{B}$. Here, $\beta$ is a random bit. $\mathcal{B}$ gives $C^{\star}$ to $\mathcal{A}$. In PhASE2, $\mathcal{B}$ deals with key-extraction queries made by $\mathcal{A}$ as in PHASE1. Finally, $\mathcal{B}$ outputs the same bit as $\mathcal{A}$. We check that $\mathcal{B}$ has not made any prohibited key-extraction query. This holds because we have $R_{f_{\mathrm{p}}\left(N^{\prime}\right)}^{\mathrm{F}}\left(f_{\mathrm{e}}\left(X^{\prime \star}\right), f_{\mathrm{k}}\left(Y^{\prime}\right)\right)=R_{N^{\prime}}^{\mathrm{F}^{\prime}}\left(X^{\prime \star}, Y^{\prime}\right)=0$ for all $Y^{\prime}$ queried by $\mathcal{A}$. It is straightforward to see that the simulation by $\mathcal{B}$ is perfect and $\mathcal{B}$ has exactly the same advantage as $\mathcal{A}$.

\section{B Key Delegation Algorithm}

In Section 3, we showed that KP-ABE scheme can be converted into DSE scheme without delegation. The conversion is completely generic and can be applicable to many existing schemes. Here, we show that it is possible to add key delegation algorithm for resulting schemes in some cases. To capture such cases, we define the following template for monotonic KP-ABE. If the original KP-ABE scheme is an instance of the template, we can add key delegation algorithm to the DSE scheme obtained by our conversion.

Let $\mathbb{G}, \mathbb{G}_{T}$ be underlying bilinear groups of order $p$. The universe of attribute is denoted by $\mathcal{U}$.

$\operatorname{Setup}(\lambda, N)$ : Given a security parameter $\lambda \in \mathbb{N}$ and some bounds on parameters $N \in \mathbb{Z}$, the algorithm selects bilinear groups $\left(\mathbb{G}, \mathbb{G}_{T}\right)$ of prime order $p>2^{\lambda}$ and a generator $g \stackrel{s}{\leftarrow} \mathbb{G}$. It computes $e(g, g)^{\alpha}$ for a random $\alpha \stackrel{\$}{\leftarrow} \mathbb{Z}_{p}$ and sets parameters pp. We assume that $g$ is included in pp. The master secret key consists of $\mathrm{msk}=\alpha$ while the master public key is $\mathrm{mpk}=\left(e(g, g)^{\alpha}, \mathrm{pp}\right)$.

We assume that pp defines publicly computable functions $f_{0}: \mathcal{U} \rightarrow \mathbb{G}^{\ell^{\prime}}, f_{1}: \mathcal{U} \rightarrow \mathbb{G}$.

KeyGen $(\mathrm{msk}, \mathrm{mpk},(\mathbf{L}, \rho))$ : The input to the algorithm is the master secret key msk, master public key $\mathrm{mpk}$, and a monotone span program $(\mathbf{L}, \rho)$. Let $\mathbf{L}$ be an $\ell \times m$ matrix. First, it picks random $\mathbf{s}[2], \ldots, \mathbf{s}[m], r_{1}, \ldots, r_{\ell} \stackrel{\$}{\leftarrow} \mathbb{Z}_{p}$ and sets the vector $\mathbf{s}=(\alpha, \mathbf{s}[2], \ldots, \mathbf{s}[m])^{\top}$. Then, it computes shares of $\alpha$ for $\rho(i)$ by $\lambda_{i}=\mathbf{L}_{i} \cdot \mathbf{s}$ for $i \in[1, \ell]$. Here, $\mathbf{L}_{i}$ is $i$-th row of $\mathbf{L}$. It then outputs private key

$$
\mathrm{sk}_{(\mathbf{L}, \rho)}=\left\{D_{i, 0}=f_{0}(\rho(i))^{r_{i}}, \quad D_{i, 1}=g^{\lambda_{i}} \cdot f_{1}(\rho(i))^{r_{i}}\right\}_{i \in[1, \ell]} .
$$

Example. Many existing KP-ABE schemes such as [25, 4, 28, 39] can be captured by the above template. For example, unbounded monotonic KP-ABE scheme in [39] can be captured by defining $\mathrm{pp}, f_{0}, f_{1}$ as

$$
\mathrm{pp}=(g, u, h, w), \quad f_{0}(\mathrm{Att})=\left(u^{\mathrm{Att}} \cdot h, g\right), \quad f_{1}(\mathrm{Att})=w .
$$

Monotonic KP-ABE scheme with constant-size ciphertexts in [4] can be captured by defining pp, $f_{0}, f_{1}$ as

$$
\mathrm{pp}=\left(g,\left\{h_{i}\right\}_{i \in[0, \bar{k}+1]}\right), \quad f_{0}(\mathrm{Att})=\left(\left\{h_{1}^{-\mathrm{Att}^{i-1}} \cdot h_{i}\right\}_{i \in[2, \bar{k}+1]}, g\right), \quad f_{1}(\mathrm{Att})=h_{0} .
$$

If the setup and key generation algorithm of KP-ABE scheme $\Pi=\{$ Setup, KeyGen, Encrypt, Decrypt $\}$ satisfy the template, one can add key delegation algorithm to a DSE scheme $\Pi^{\prime}$ that is obtained by applying our conversion in Section 3 to the KP-ABE scheme $\Pi$.

Before showing key delegation algorithm, we first define ReRand algorithm for $\Pi$ that re-randomizes private key. This will be used as a subroutine in the key delegation algorithm of the DSE scheme $\Pi^{\prime}$. 
$\operatorname{ReRand}\left(\operatorname{mpk} \mathbf{s k}_{(\mathbf{L}, \rho)},(\mathbf{L}, \rho)\right)$ : It takes as input mpk, a private key sk $(\mathbf{L}, \rho)$, and a span program $(\mathbf{L}, \rho)$ and outputs re-randomized private key $\operatorname{sk}_{(\mathbf{L}, \rho)}^{\prime}$ for the same span program. Let $\mathbf{L}$ be $\ell \times m$ matrix. It first parse the private key as $\left\{D_{i, 0}, D_{i, 1}\right\}_{i \in[1, \ell]}$. Then it picks $\mathbf{s}^{\prime}[2], \ldots, \mathbf{s}^{\prime}[m], r_{1}^{\prime}, \ldots, r_{\ell}^{\prime} \stackrel{\$}{\leftarrow} \mathbb{Z}_{p}$ and sets $\lambda_{i}^{\prime}=\mathbf{L}_{i} \cdot \mathbf{s}^{\prime}$ for each $i \in[1, \ell]$ where $\mathbf{s}^{\prime}=\left(0, \mathbf{s}^{\prime}[2], \ldots, \mathbf{s}^{\prime}[m]\right)^{\top}$ and $\mathbf{L}_{i}$ is the $i$-th row of $\mathbf{L}$. Finally, it outputs

$$
\left\{D_{i, 0}^{\prime}=D_{i, 0} \cdot f_{0}(\rho(i))^{r_{i}^{\prime}}, \quad D_{i, 1}^{\prime}=D_{i, 1} \cdot g^{\lambda_{i}^{\prime}} \cdot f_{1}(\rho(i))^{r_{i}^{\prime}}\right\}_{i \in[1, \ell]} .
$$

We have following claim.

Claim 1. For all $\lambda$, (mpk, msk $) \leftarrow \operatorname{Setup}(\lambda),(\mathbf{L}, \rho)$, and $\mathbf{s k}_{(\mathbf{L}, \rho)} \leftarrow \operatorname{KeyGen}($ msk, mpk, $(\mathbf{L}, \rho))$, we have the following distributions are identical:

$$
\operatorname{ReRand}\left(\operatorname{mpk}_{\mathbf{L}} \mathrm{sk}_{(\mathbf{L}, \rho)},(\mathbf{L}, \rho)\right) \approx \operatorname{KeyGen}(\mathrm{mpk}, \mathrm{msk},(\mathbf{L}, \rho)) \text {. }
$$

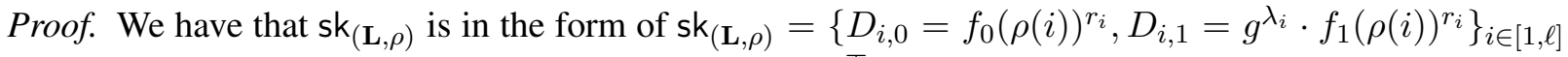
where $\lambda_{i}=\mathbf{L}_{i} \cdot \mathbf{s}$ for some $\mathbf{s}=(\alpha, \mathbf{s}[2], \ldots, \mathbf{s}[m])^{\top} \in \mathbb{Z}_{p}^{m}$ and $\left\{r_{i}\right\}_{i \in[1, \ell]}$. Then, the output of $\operatorname{ReRand}\left(\mathrm{mpk}^{\mathrm{sk}} \mathbf{s k}_{(\mathbf{L}, \rho)},(\mathbf{L}, \rho)\right)$ is $\left\{\tilde{D}_{i, 0}=f_{0}(\rho(i))^{r_{i}+r_{i}^{\prime}}, \tilde{D}_{i, 1}=g^{\lambda_{i}+\lambda_{i}^{\prime}} \cdot f_{1}(\rho(i))^{r_{i}+r_{i}^{\prime}}\right\}_{i \in[1, \ell]}$. We have that $\lambda_{i}^{\prime}+\lambda_{i}=\mathbf{L}_{i} \cdot\left(\mathbf{s}+\mathbf{s}^{\prime}\right)$ and $\mathbf{s}+\mathbf{s}^{\prime}=\left(\alpha, \mathbf{s}[2]+\mathbf{s}^{\prime}[2], \ldots, \mathbf{s}[m]+\mathbf{s}^{\prime}[m]\right)^{\top}$. Here, $\mathbf{s}[i]+\mathbf{s}^{\prime}[i]$ for $i \in[2, m]$ and $r_{i}+r_{i}^{\prime}$ for $i \in[1, m]$ are uniformly distributed over $\mathbb{Z}_{p}$ and independent from the randomness used to generate $\mathbf{s k}_{(\mathbf{L}, \rho)}$ (namely, $\{\mathbf{s}[i]\}_{i \in[2, m]}$ and $\left\{r_{i}\right\}_{i \in[1, m]}$ ). Thus the output distribution of $\operatorname{ReRand}\left(\mathrm{mpk} \mathrm{sk}_{(\mathbf{L}, \rho)},(\mathbf{L}, \rho)\right)$ is the same as that of KeyGen(mpk, msk, $\left.(\mathbf{L}, \rho)\right)$.

Now we define key delegation algorithm KeyDel ${ }^{\prime}$ for $\Pi^{\prime}$. In the following, we let $n^{\prime}=(2 n \kappa+1)(n+1)$.

$\operatorname{KeyDel}^{\prime}\left(\mathrm{mpk}, \mathrm{sk}_{\left(\mathbf{y}_{0}, \mathbf{Y}\right)},\left(\mathbf{y}_{0}, \mathbf{Y}\right),\left(\hat{\mathbf{y}}_{0}, \hat{\mathbf{Y}}\right)\right)$ : It takes as input the master public key mpk, a private key $\operatorname{sk}_{\left(\mathbf{y}_{0}, \mathbf{Y}\right)}$ for $\left(\mathbf{y}_{0}, \mathbf{Y}\right) \in \mathbb{Z}_{p}^{n} \times \mathbb{Z}_{p}^{n \times d}$, and $\left(\hat{\mathbf{y}}_{0}, \hat{\mathbf{Y}}\right) \in \mathbb{Z}_{p}^{n} \times \mathbb{Z}_{p}^{n \times \hat{d}}$ such that $\hat{\mathbf{y}}_{0}+\operatorname{span}(\hat{\mathbf{Y}}) \subseteq \mathbf{y}_{0}+$ $\operatorname{span}(\mathbf{Y})$. Here, $\operatorname{sk}_{\left(\mathbf{y}_{0}, \mathbf{Y}\right)}$ is generated by sk $\left(\mathbf{y}_{0}, \mathbf{Y}\right) \leftarrow \operatorname{KeyGen}^{\prime}\left(\mathrm{msk}, \mathrm{mpk},\left(\mathbf{y}_{0}, \mathbf{Y}\right)\right)=\operatorname{KeyGen}(\mathrm{msk}$, mpk, $(\mathbf{L}, \rho))$ such that $(\mathbf{L}, \rho)=f_{\mathrm{k}}^{\operatorname{DSE} \rightarrow \mathrm{KP}}\left(\mathbf{y}_{0}, \mathbf{Y}\right)$. The key delegation algorithm first parses the key as $\operatorname{sk}_{\left(\mathbf{y}_{0}, \mathbf{Y}\right)}=\left\{D_{i, 0}, D_{i, 1}\right\}_{i \in\left[1, n^{\prime}+d+1\right]}$ and computes $\mathbf{t}=(\mathbf{t}[1], \ldots, \mathbf{t}[d])^{\top} \in \mathbb{Z}_{p}^{d}$ and $\mathbf{T}=$ $\left(T_{i, j}\right)_{(i, j) \in[1, d] \times[1, \hat{d}]} \in \mathbb{Z}_{p}^{d \times \hat{d}}$ such that $\hat{\mathbf{y}}_{0}=\mathbf{y}_{0}+\mathbf{Y t}$ and $\hat{\mathbf{Y}}=\mathbf{Y T}$. Such $\mathbf{t}$ and $\mathbf{T}$ exist because $\hat{\mathbf{y}}_{0}+\operatorname{span}(\hat{\mathbf{Y}}) \subseteq \mathbf{y}_{0}+\operatorname{span}(\mathbf{Y})$ and can be computed efficiently. Then it sets $\tilde{D}_{j, b}$ for $j \in\left[1, n^{\prime}+\hat{d}+1\right]$ and $b \in\{0,1\}$ as

$$
\tilde{D}_{j, b}=\left\{\begin{array}{lll}
D_{1, b} \cdot \prod_{i \in[1, d]} D_{1+i, b}^{\mathbf{t}[i]} & \text { if } \quad j=1 \\
\prod_{i \in[1, d]} D_{1+i, b}^{T, j-1} & \text { if } \quad j \in[2, \hat{d}+1] \\
D_{j-\hat{d}+d, b} & \text { if } j \in\left[\hat{d}+2, n^{\prime}+\hat{d}+1\right]
\end{array}\right.
$$

It runs ReRand(mpk, $\left.\left\{\tilde{D}_{i, 0}, \tilde{D}_{i, 1}\right\}_{i \in\left[1, n^{\prime}+\hat{d}+1\right]},(\hat{\mathbf{L}}, \hat{\rho})\right) \rightarrow\left\{D_{i, 0}^{\prime}, D_{i, 1}^{\prime}\right\}_{i \in\left[1, n^{\prime}+\hat{d}+1\right]}$ and outputs sk ${ }_{\left(\hat{\mathbf{y}}_{0}, \hat{\mathbf{Y}}\right)}$ $=\left\{D_{i, 0}^{\prime}, D_{i, 1}^{\prime}\right\}_{i \in\left[1, n^{\prime}+\hat{d}+1\right]}$ where $(\hat{\mathbf{L}}, \hat{\rho})=f_{\mathrm{k}}^{\mathrm{DSE} \rightarrow \mathrm{KP}}\left(\hat{\mathbf{y}}_{0}, \hat{\mathbf{Y}}\right)$.

The following claim indicates that the key delegation algorithm described above correctly works.

Claim 2. For all $\lambda, n$, (mpk, msk $) \leftarrow \operatorname{Setup}^{\prime}(\lambda, n), \mathbf{y}_{0}, \mathbf{Y}, \hat{\mathbf{y}}_{0}, \hat{\mathbf{Y}}$ such that $\hat{\mathbf{y}}_{0}+\operatorname{span}(\hat{\mathbf{Y}}) \subseteq \mathbf{y}_{0}+$ $\operatorname{span}(\mathbf{Y})$, and $\mathrm{sk}_{\left(\mathbf{y}_{0}, \mathbf{Y}\right)} \leftarrow \mathrm{KeyGen}^{\prime}\left(\mathrm{msk}, \mathrm{mpk},\left(\mathbf{y}_{0}, \mathbf{Y}\right)\right)$, we have the following distributions are identical:

$$
\operatorname{KeyDel}^{\prime}\left(\mathrm{mpk}, \operatorname{sk}_{\left(\mathbf{y}_{0}, \mathbf{Y}\right)},\left(\mathbf{y}_{0}, \mathbf{Y}\right),\left(\hat{\mathbf{y}}_{0}, \hat{\mathbf{Y}}\right)\right) \approx \operatorname{KeyGen}^{\prime}\left(\mathrm{mpk}, \mathrm{msk},\left(\hat{\mathbf{y}}_{0}, \hat{\mathbf{Y}}\right)\right)
$$


Proof. Let $\left\{\tilde{D}_{i, 0}, \tilde{D}_{i, 1}\right\}_{i \in\left[1, n^{\prime}+\hat{d}+1\right]}$ be defined as above which is computed from $\mathbf{s k}_{(\mathbf{L}, \rho)}=\left\{D_{i, 0}=\right.$ $\left.f_{0}(\rho(i))^{r_{i}}, D_{i, 1}=g^{\lambda_{i}} \cdot f_{1}(\rho(i))^{r_{i}}\right\}_{i \in\left[1, n^{\prime}+d+1\right]}$ where $\lambda_{i}=\mathbf{L}_{i} \cdot \mathbf{s}$ for some $\mathbf{s}=\left(\alpha, \mathbf{s}[2], \ldots, \mathbf{s}\left[m^{\prime}\right]\right)^{\top} \in \mathbb{Z}_{p}^{m^{\prime}}$ and $\left\{r_{i} \in \mathbb{Z}_{p}\right\}_{i \in[1, \ell]}$. Here, $m^{\prime}=(n \kappa+1)(n+1)+1$. We will show that $\operatorname{Pr}\left[\left\{\tilde{D}_{i, 0}, \tilde{D}_{i, 1}\right\}_{i \in\left[1, n^{\prime}+\hat{d}+1\right]}=\right.$ $\operatorname{KeyGen}(\mathrm{msk}, \mathrm{mpk},(\hat{\mathbf{L}}, \hat{\rho}))]>0$. Then, the output of KeyDel defined as above is correctly distributed due to Equation (8).

For $j \in\left[\hat{d}+2, n^{\prime}+\hat{d}+1\right]$ and $b \in\{0,1\}$, we have

$$
\tilde{D}_{j, b}=g^{b \cdot \mathbf{L}_{j-\hat{d}+d} \cdot \mathbf{s}} \cdot f_{b}(\rho(j-\hat{d}+d))^{r_{j-\hat{d}+d}}=g^{b \cdot \hat{\mathbf{L}}_{j} \cdot \mathbf{s}} \cdot f_{b}(\hat{\rho}(j))^{r_{j}^{\prime}}
$$

where $r_{j}^{\prime}=r_{j-\hat{d}+d}$ for $j \in\left[\hat{d}+2, n^{\prime}+\hat{d}+1\right]$. The second equation above holds by the definition of $(\mathbf{L}, \rho)$ and $(\hat{\mathbf{L}}, \hat{\rho})$. For $j \in[2, \hat{d}+1]$ and $b \in\{0,1\}$, we have that

$$
\begin{aligned}
\tilde{D}_{j, b} & =\prod_{i \in[1, d]} D_{1+i, b}^{T_{i, j-1}}=\prod_{i \in[1, d]}\left(g^{b \cdot \mathbf{L}_{1+i} \cdot \mathbf{s}} \cdot f_{b}(\rho(1+i))^{r_{1+i}}\right)^{T_{i, j-1}} \\
& =\left(g^{\sum_{i \in[1, d]} T_{i, j-1} \mathbf{L}_{1+i} \cdot \mathbf{s}}\right)^{b} \cdot f_{b}(\mathrm{D})^{\sum_{i \in[1, d]} r_{1+i} T_{i, j-1}} \\
& \left.=\left(g^{\sum_{i \in[1, d]} T_{i, j-1} \mathbf{y}_{i}^{\top} \cdot \mathbf{s}^{\prime}}\right)^{b} \cdot f_{b}(\mathrm{D})^{r_{j}^{\prime}} \quad \text { (By the structure of } \mathbf{L} .\right) \\
& \left.=\left(g^{\hat{\mathbf{y}}_{j-1}^{\top} \cdot \mathbf{s}^{\prime}}\right)^{b} \cdot f_{b}(\mathrm{D})^{r_{j}^{\prime}} \quad \text { (By the definition of } \mathbf{T} .\right) \\
& \left.=\left(g^{\hat{\mathbf{L}}_{j} \cdot \mathbf{s}}\right)^{b} \cdot f_{b}(\hat{\rho}(j))^{r_{j}^{\prime}} \quad \text { (By the structure of } \hat{\mathbf{L}} .\right)
\end{aligned}
$$

In the above, $r_{j}^{\prime}=\sum_{i \in[1, d]} r_{1+i} T_{i, j-1}$ for $j \in[2, \hat{d}+1], \mathbf{s}^{\prime}=(\mathbf{s}[3], \ldots, \mathbf{s}[n+2])^{\top}$, and $\mathbf{y}_{i}$ is the $i$-th column of $\mathbf{Y}$. We also have

$$
\begin{aligned}
\tilde{D}_{1, b} & =D_{1, b} \cdot \prod_{i \in[1, d]} D_{1+i, b}^{\mathbf{t}[i]}=g^{b \cdot \mathbf{L}_{1} \cdot \mathbf{s}} \cdot f_{b}(\rho(1))^{r_{1}} \cdot \prod_{i \in[1, d]}\left(g^{b \cdot \mathbf{L}_{1+i} \cdot \mathbf{s}} \cdot f_{b}(\rho(1+i))^{r_{1+i}}\right)^{\mathbf{t}[i]} \\
& =\left(g^{\left(\mathbf{L}_{1}+\sum_{i \in[1, d]} \mathbf{t}[i] \mathbf{L}_{1+i}\right) \cdot \mathbf{s}}\right)^{b} \cdot f_{b}(\mathrm{D})^{r_{1}+\sum_{i \in[1, d]} r_{1+i} \mathbf{t}[i]} \\
& =\left(g^{\alpha+\mathbf{s}[2]+\left(\mathbf{y}_{0}^{\top}+\sum_{i \in[1, d]} \mathbf{t}[i] \mathbf{y}_{i}^{\top}\right) \cdot \mathbf{s}^{\prime}}\right)^{b} \cdot f_{b}(\mathrm{D})^{r_{1}^{\prime}} \quad(\text { By the structure of } \mathbf{L} .) \\
& =\left(g^{\alpha+\mathbf{s}[2]+\hat{\mathbf{y}}_{0}^{\top} \cdot \mathbf{s}^{\prime}}\right)^{b} \cdot f_{b}(\mathrm{D})^{r_{1}^{\prime}} \quad(\text { By the definition of } \mathbf{t} .) \\
& =\left(g^{\hat{\mathbf{L}}_{1} \cdot \mathbf{s}}\right)^{b} \cdot f_{b}(\hat{\rho}(1))^{r_{1}^{\prime}} \quad(\text { By the structure of } \hat{\mathbf{L}} .)
\end{aligned}
$$

In the above, $r_{1}^{\prime}=r_{1}+\sum_{i \in[1, d]} r_{1+i} \mathbf{t}[i]$.

To sum up, we have $\left(\tilde{D}_{i, 0}, \tilde{D}_{i, 1}\right)=\left(f_{0}(\hat{\rho}(i))^{r_{i}^{\prime}}, g^{\hat{\mathbf{L}}_{i} \cdot \mathbf{s}} \cdot f_{1}(\hat{\rho}(i))^{r_{i}^{\prime}}\right)$ for $i \in\left[1, n^{\prime}+\hat{d}+1\right]$. This implies that $\left(\tilde{D}_{i, 0}, \tilde{D}_{i, 1}\right)$ can be seen as a private key for $(\hat{\mathbf{L}}, \hat{\rho})$ which is generated by randomness $\left\{r_{i}^{\prime}\right\}_{i \in\left[n^{\prime}+\hat{d}+1\right]}$ and $\{\mathbf{s}[i]\}_{i \in\left[2, m^{\prime}\right]}$. Thus we have

$$
\operatorname{Pr}\left[\left\{\tilde{D}_{i, 0}, \tilde{D}_{i, 1}\right\}_{i \in\left[1, n^{\prime}+\hat{d}+1\right]}=\operatorname{KeyGen}(\mathrm{msk}, \mathrm{mpk},(\hat{\mathbf{L}}, \hat{\rho}))\right]>0
$$

as desired. 


\section{From K(C)ASP to KP(CP)-ABE}

In Section 5, we showed that monotonic $\mathrm{KP}(\mathrm{CP})-\mathrm{ABE}$ can be converted into $\mathrm{K}(\mathrm{C}) \mathrm{ASP}$. In this section, we also show the converse direction. Namely, we show that KASP can be converted into non-monotonic $\mathrm{KP}-\mathrm{ABE}$ with large universe (which trivially implies monotonic KP-ABE for small universe). The result in this section together with that in Section 5 imply that these two notions are in fact equivalent in the sense that one implies the other. These results also imply that monotonic KP-ABE for small universe and nonmonotonic KP-ABE with large universe are equivalent. We note that we can obtain similar implications for the case of CP-ABE and CASP by swapping key and ciphertext attributes in the following.

\section{C.1 The Conversion}

We construct non-monotonic KP-ABE with large universe for $N=(\bar{k},-,-,-)$ from KASP with dimension $\bar{k}+1$. As in Section 3, we define appropriate maps $f_{\mathrm{p}}^{\mathrm{KP} \rightarrow \mathrm{KASP}}, f_{\mathrm{e}}^{\mathrm{KP} \rightarrow \mathrm{KASP}}, f_{\mathrm{k}}^{\mathrm{KP} \rightarrow \mathrm{KASP}}$. At first, we define $f_{\mathrm{p}}^{\mathrm{KP} \rightarrow \mathrm{KASP}}(N)=\bar{k}+1$. We assume that the universe of attributes is $\mathbb{Z}_{p}$. This restriction can be easily removed by using collision resistant hash. The idea for the conversion is very similar to that in Section 4.

Next we define $f_{\mathrm{e}}^{\mathrm{KP} \rightarrow \mathrm{KASP}}$ that takes as input a set of attributes $S=\left(S_{1}, \ldots, S_{k}\right) \subset \mathbb{Z}_{p}$ such that $k \leq \bar{k}$ and outputs a vector $\mathbf{q}_{S}=\left(\mathbf{q}_{S}[1], \ldots, \mathbf{q}_{S}[\bar{k}+1]\right)^{\top} \in \mathbb{Z}_{p}^{\bar{k}+1}$ which is defined as a coefficient vector from

$$
Q_{S}[Z]=\sum_{i=1}^{k+1} \mathbf{q}_{S}[i] \cdot Z^{i-1}=\prod_{i=1}^{k}\left(Z-S_{i}\right)
$$

where, if $k<\bar{k}$, the coordinates $\mathbf{q}_{S}[k+2], \ldots, \mathbf{q}_{S}[\bar{k}+1]$ are all set to 0 . We note that for $x \in \mathbb{Z}_{p}$, $Q_{S}(x)=\prod_{i=1}^{k}\left(x-S_{i}\right)=0$ iff $x \in S$. This property will be used later.

Next we define $f_{\mathrm{k}}^{\mathrm{KP} \rightarrow \mathrm{KASP}}$ that takes as input a span program $\left(\mathbf{L} \in \mathbb{Z}_{p}^{\ell \times m}, \rho\right)$ and outputs an arithmetic $\operatorname{span} \operatorname{program}\left(\mathbf{Y}, \mathbf{Z}, \rho^{\prime}\right)$. Let the size of $\mathbf{L}$ be $\left(\ell_{0}+\ell_{1}\right) \times m$ where $\ell=\ell_{0}+\ell_{1}$ and let $\bar{\ell}=\max \left\{\ell_{0}, \ell_{1}\right\}$. Without loss of generality, we assume that the first $\ell_{0}$ rows of the matrix are associated with positive attributes and the last $\ell_{1}$ rows with negative attributes by a map $\rho$. We denote $\mathbf{L}$ as $\mathbf{L}=\left[\mathbf{L}_{0} ; \mathbf{L}_{1}\right]$ using matrices $\mathbf{L}_{0} \in \mathbb{Z}_{p}^{\ell_{0} \times m}$ and $\mathbf{L}_{1} \in \mathbb{Z}_{p}^{\ell_{1} \times m}$. $\mathbf{Y}$ and $\mathbf{Z}$ are defined as

$$
\begin{aligned}
& \mathbf{Y}^{\top}=(\overbrace{}^{m} \mathbf{I}_{\ell} \otimes \mathbf{1}_{\bar{k}+1} \overbrace{\left(\ell_{0}+2 \ell_{1}\right) \times(2(\bar{k}+2) \ell+m)}^{(\bar{k}+1) \bar{\ell}} \mathbf{I}_{\ell} \otimes \mathbf{1}_{\bar{k}+1} \overbrace{}^{(\bar{k}+1) \bar{\ell}}) \in \mathbb{Z}_{p}^{\left(\ell_{0}+2 \ell_{1}+2(\bar{k}+1) \bar{\ell}\right) \times(2(\bar{k}+2) \ell+m)} . \\
& \mathbf{Z}^{\top}=\left(\begin{array}{ccccc}
\mathbf{L}_{0} & \overbrace{}^{\bar{\ell}} & \mathbf{G}_{0} & & \\
\mathbf{L}_{1} & & \mathbf{I}_{\ell_{1}} & \overbrace{}^{\bar{\ell}-\ell_{1}} & \\
& & \mathbf{I}_{(\bar{k}+1) \bar{\ell}} & & \mathbf{G}_{1} \\
& & & & \mathbf{I}_{(\bar{k}+1) \bar{\ell}}
\end{array}\right) \in \mathbb{Z}_{p}^{\left(\ell_{0}+2 \ell_{1}+2(\bar{k}+1) \bar{\ell}\right) \times(2(\bar{k}+2) \ell+m)} .
\end{aligned}
$$

Here, $\mathbf{G}_{b} \in \mathbb{Z}_{p}^{\ell_{b} \times \bar{\ell}(\bar{k}+1)}$ for $b \in\{0,1\}$ are defined as Equation (4).

We also define $\rho^{\prime}:\left[\ell_{0}+2 \ell_{1}+2(\bar{k}+1) \bar{\ell}\right] \rightarrow[1, \bar{k}+1]$ as follows. 
- If $i \in\left[1, \ell_{0}+2 \ell_{1}\right]$, we set $\rho^{\prime}(i)=1$.

- Else, we have $i \in\left[\ell_{0}+2 \ell_{1}+1, \ell_{0}+2 \ell_{1}+2(\bar{k}+1) \bar{\ell}\right]$. We then set $\rho^{\prime}(i)=i^{\prime}$ where $i^{\prime} \in[1, \bar{k}+1]$ is a unique integer that satisfies

$$
i-\left(\ell_{0}+2 \ell_{1}\right) \equiv i^{\prime} \bmod (\bar{k}+1)
$$

\section{C.2 Correctness of the Conversion}

By combining the following theorem with Lemma 1, we can see that monotonic KP-ABE with large universe can be constructed from KASP.

Theorem 4. For any span program $(\mathbf{L}, \rho)$ and $S$ such that $|S| \leq \bar{k}$, we have that

$$
R^{\mathrm{KASP}}\left(\mathbf{x},\left(\mathbf{Y}, \mathbf{Z}, \rho^{\prime}\right)\right)=1 \Leftrightarrow R^{\mathrm{KP}}(S,(\mathbf{L}, \rho))=1
$$

where $\mathbf{x}=f_{\mathrm{e}}^{\mathrm{KP} \rightarrow \mathrm{KASP}}(S)$ and $\left(\mathbf{Y}, \mathbf{Z}, \rho^{\prime}\right)=f_{\mathrm{k}}^{\mathrm{KP} \rightarrow \mathrm{KASP}}(\mathbf{L}, \rho)$.

Proof. Let $\mathbf{W}$ be a matrix such that the $i$-th column is $\mathbf{x}\left[\rho^{\prime}(i)\right] \cdot \mathbf{y}_{i}+\mathbf{z}_{i}$ where $\mathbf{y}_{i}$ and $\mathbf{z}_{i}$ are the $i$-th column of $\mathbf{Y}$ and $\mathbf{Z}$ respectively. Then, from the definition of $\rho^{\prime}$, we have that

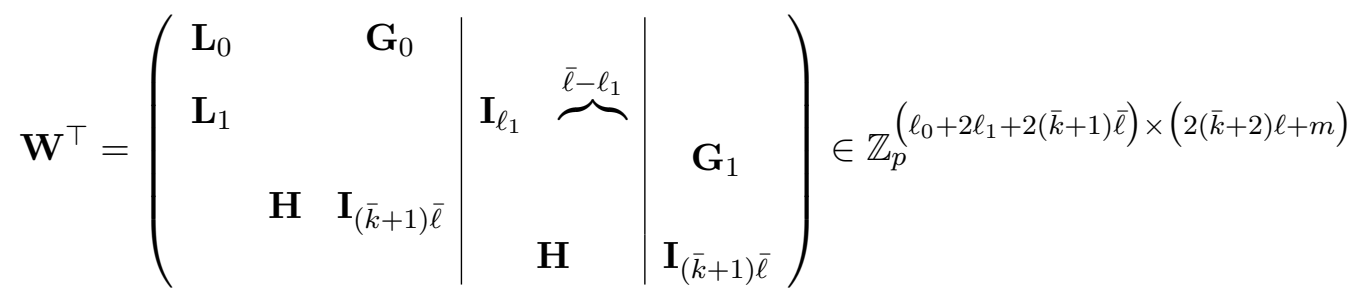

where $\mathbf{H}=\mathbf{I}_{\bar{\ell}} \otimes \mathbf{q}_{S} \in \mathbb{Z}_{p}^{(\bar{k}+1) \bar{\ell} \times \bar{\ell}}$. We divide the matrix $\mathbf{W}^{\top}$ into two parts as $\mathbf{W}=\left(\mathbf{W}_{0}, \mathbf{W}_{1}\right)$ where $\mathbf{W}_{0} \in \mathbb{Z}_{p}^{(2(\bar{k}+2) \ell+m) \times\left(\ell_{0}+2 \ell_{1}\right)}$ and $\mathbf{W}_{1} \in \mathbb{Z}_{p}^{(2(\bar{k}+2) \ell+m) \times 2(\bar{k}+1) \bar{\ell}}$. Note that $\mathbf{W}_{0}$ and $\mathbf{W}_{1}$ defined here have exactly the same structure as $\mathbf{X}$ and $\mathbf{Y}$ defined in Section 4 respectively. We also let $I \subset[1, \ell(=$ $\left.\left.\ell_{0}+\ell_{1}\right)\right]$ be $I=\{i \mid \rho(i) \in S\} . \mathbf{L}_{I}$ is defined as the sub-matrix of $\mathbf{L}$ formed by rows whose index is in $I$. Then, we have

$$
\begin{aligned}
\mathbf{e}_{1} \in \operatorname{span}\left(\mathbf{L}_{I}^{\top}\right) \Leftrightarrow & \quad\left(-\mathbf{e}_{1}+\operatorname{span}\left(\mathbf{W}_{0}\right)\right) \cap \operatorname{span}\left(\mathbf{W}_{1}\right) \neq \emptyset \\
& \quad(\text { By exactly the same analysis as in the proof of Theorem } 2 .) \\
\Leftrightarrow & \mathbf{e}_{1} \in \operatorname{span}(\mathbf{W}) .
\end{aligned}
$$

By the definition of $R^{\mathrm{KASP}}$ and $R^{\mathrm{KP}}$, this concludes the proof of the theorem.

\section{Our Attribute-Based Signature Scheme}

Here, we define predicate signature (PS) which is a signature analogue of predicate encryption. Attributebased signature (ABS) can be captured as a special case of PS in which a private key is associated with a set of attributes while a signature is associated with a policy. In this section, we construct a PS scheme that is dual of ABS: a private key is associated with a policy and a signature with a set. By applying our KPABE-to-CP-ABE conversion technique developed in Section 3 and 4 to the PS scheme, we obtain the first ABS with constant-size signatures that supports non-monotone span programs. Note that previous ABS schemes with constant-size signatures $[27,12]$ only support threshold predicate. Our result significantly improves the expressibility. 


\section{D.1 Predicate Signature}

Here, we define predicate signature. Let $R=\left\{R_{N}: A_{N} \times B_{N} \rightarrow\{0,1\} \mid N \in \mathbb{N}^{c}\right\}$ be a relation family where $A_{N}$ and $B_{N}$ denote "verification attribute" and "key attribute" spaces and $c$ is some fixed constant. The index $N=\left(n_{1}, n_{2}, \ldots, n_{c}\right)$ of $R_{N}$ denotes the numbers of bounds for corresponding parameters. A predicate signature (PS) scheme $\Sigma$ for $R_{N}$ is defined by the following algorithms:

$\operatorname{Setup}(\lambda, N) \rightarrow(\mathrm{mpk}, \mathrm{msk})$ : The setup algorithm is defined as the same as PE.

KeyGen $(m s k, m p k, Y) \rightarrow s k_{Y}$ : The key generation algorithm is defined as the same as PE.

$\operatorname{Sign}\left(\mathrm{mpk}, \mathrm{M}, X, \mathrm{sk}_{Y}, Y\right) \rightarrow \sigma$ : The input to the signing algorithm is the master public key mpk, a message $\mathrm{M}$, verification attribute $X$, private $\mathrm{key} \mathrm{k}_{Y}$, and key attribute $Y$. It outputs a signature $\sigma$ if $R_{N}(X, Y)=1$ and otherwise $\perp$.

Verify $(\mathrm{mpk}, \mathrm{M}, \sigma, X) \rightarrow 1$ or 0 : The verification algorithm takes as input a master public key mpk, the message $\mathrm{M}$, a signature $\sigma$, and a verification attribute $X \in A_{N}$. It outputs 1 if the signature is deemed valid and 0 otherwise. We assume that verification algorithm is deterministic.

We require correctness: that is, for all $\lambda, N$, (mpk, msk) $\leftarrow \operatorname{Setup}(\lambda, N), X \in A_{N}, Y \in B_{N}$ such that $R(X, Y)=1, \mathrm{M}$ in specified message space, $\mathrm{sk}_{Y} \leftarrow$ KeyGen (msk, mpk, $\left.Y\right)$, and $\sigma \leftarrow \operatorname{Sign}(\mathrm{mpk}, \mathrm{M}, X$, $\left.\mathrm{sk}_{Y}, Y\right)$, Verify $(\mathrm{mpk}, \mathrm{M}, \sigma, X)=1$ holds.

For security of PS, we require privacy and unforgeability.

Privacy. We say that the PS scheme has complete privacy if for all $\lambda, N,(\mathrm{mpk}, \mathrm{msk}) \leftarrow \operatorname{Setup}(\lambda, N)$, $X \in A_{N}, Y_{0}, Y_{1} \in B_{N}$ satisfying $R_{N}\left(X, Y_{0}\right)=R_{N}\left(X, Y_{1}\right)=1, \mathrm{M}$ in specified message space, and $\mathrm{sk}_{Y_{b}} \leftarrow \operatorname{KeyGen}\left(\mathrm{msk}, \mathrm{mpk}, Y_{b}\right)$ for $b \in\{0,1\}$, the following distributions are the same.

$$
\left\{\sigma_{0} \leftarrow \operatorname{Sign}\left(\mathrm{mpk}, \mathrm{M}, X, \mathrm{sk}_{Y_{0}}, Y_{0}\right)\right\} \approx\left\{\sigma_{1} \leftarrow \operatorname{Sign}\left(\mathrm{mpk}, \mathrm{M}, X, \mathrm{sk}_{Y_{1}}, Y_{1}\right)\right\}
$$

Unforgeability. We now define the unforgeability for PS scheme $\Sigma$. This security notion is defined by the following game between a challenger and a forger $\mathcal{F}$.

At first, the challenger runs the setup algorithm and gives mpk to $\mathcal{F}$. Then $\mathcal{F}$ may adaptively make keyextraction queries. If $\mathcal{F}$ submits $Y \in B_{N}$ to the challenger, the challenger returns sk $\mathrm{k}_{Y} \leftarrow \operatorname{KeyGen}$ (msk, mpk, $Y) . \mathcal{F}$ may also makes signing queries. If $\mathcal{F}$ submits $(\mathrm{M}, X, Y)$ such that $R_{N}(X, Y)=1$ to the challenger, the challenger makes sk $\mathrm{s}_{Y} \leftarrow$ KeyGen $(\mathrm{msk}, \mathrm{mpk}, Y)$ and computes $\sigma \leftarrow \operatorname{Sign}\left(\mathrm{mpk}, \mathrm{M}, X, \mathrm{sk}_{Y}, Y\right)$ which is returned to $\mathcal{F}$. In the case of $R_{N}(X, Y)=0$, the challenger returns $\perp$ to $\mathcal{F}$. At last, $\mathcal{F}$ outputs a forgery $\left(\mathrm{M}^{\star}, X^{\star}, \sigma^{\star}\right)$. We say that $\mathcal{F}$ succeeds if $\operatorname{Verify}\left(\mathrm{mpk}, \mathrm{M}^{\star}, \sigma^{\star}, X^{\star}\right) \rightarrow 1, \mathcal{F}$ never made a keyextraction query for $Y$ such that $R\left(X^{\star}, Y\right)=1$, and $\mathcal{F}$ never made a signing query for $\left(\mathrm{M}^{\star}, X^{\star}, Y\right)$ such that $R\left(X^{\star}, Y\right)=1$. The advantage of a forger $A d v_{\mathcal{F}, \Sigma}^{P S}$ is defined as the success probability of $\mathcal{F}$ in the above game. We say that $\Sigma$ satisfies adaptive-predicate and message unforgeability under chosen message attack if $A d v_{\mathcal{A}, \Pi}^{P E}$ is negligible for all probabilistic polynomial time (PPT) adversary $\mathcal{A}$.

A weaker notion called selective-predicate and adaptive-message unforgeability can be defined as in the above game with the exception that the adversary $\mathcal{A}$ has to choose $X^{\star}$ before seeing mpk but keyextraction queries, signing queries, and choice of $\mathrm{M}^{\star}$ can still be adaptive.

\section{D.2 Linear Secret Sharing Scheme}

We can construct secret sharing scheme for span program $(\mathbf{L}, \rho)$ as follows. 
Definition 1 (Linear Secret Sharing Scheme). A secret sharing scheme for span program $(\mathbf{L}, \rho)$ consists of the following two algorithms.

Share $_{\mathbf{L}, \rho}$ The algorithm takes as input $s \in \mathbb{Z}_{p}$ which is to be shared. Let $\mathbf{L}$ be an $\ell \times m$ matrix. It chooses $\mathbf{s}[2], \ldots, \mathbf{s}[m] \stackrel{s}{\leftarrow} \mathbb{Z}_{p}$ and let $\mathbf{s}=(s, \mathbf{s}[2], \ldots, \mathbf{s}[m])^{\top}$. It outputs $\mathbf{L} \cdot \mathbf{s}$ as the vector of $\ell$ shares. The share $\lambda_{i}=\left\langle\mathbf{L}_{i}, \mathbf{s}\right\rangle$ belongs to party $\rho(i)$ for $i \in[1, \ell]$, where $\mathbf{L}_{i}$ denotes the $i$-th row of $\mathbf{L}$.

$\operatorname{Recon}_{L, \pi}$ The algorithm takes as input a set $S \subseteq \mathcal{U}$. Assume that the indicator vector $\delta$ of $S$ is accepted by $(\mathbf{L}, \rho)$. Then it outputs a set of constants $\left\{\left(i, \mu_{i}\right)\right\}_{i \in I}$ which has a linear reconstruction property: $\sum_{i \in I} \mu_{i} \cdot \lambda_{i}=s$. Here, $I$ is a set of indices whose labels of corresponding rows are set to 1 by the input $\delta$.

\section{D.3 Embedding Lemma for PS}

As noted in [26], a signature analogue of Lemma 1 holds.

Lemma 2. Consider two relation families $R_{N}^{\mathrm{F}}: A_{N} \times B_{N} \rightarrow\{0,1\}$ and $R_{N^{\prime}}^{\mathrm{F}^{\prime}}: A_{N^{\prime}}^{\prime} \times B_{N^{\prime}}^{\prime} \rightarrow\{0,1\}$, which is parametrized by $N \in \mathbb{N}^{c}$ and $N^{\prime} \in \mathbb{N}^{c^{\prime}}$ respectively. Suppose that there exist efficient mappings $f_{\mathrm{p}}: \mathbb{Z}^{c^{\prime}} \rightarrow \mathbb{Z}^{c}, f_{\mathrm{e}}: A_{N^{\prime}}^{\prime} \rightarrow A_{f_{\mathrm{p}}\left(N^{\prime}\right)}$, and $f_{\mathrm{k}}: B_{N^{\prime}}^{\prime} \rightarrow B_{f_{\mathrm{p}}\left(N^{\prime}\right)}$ such that $f_{\mathrm{e}}$ is injective and

$$
R_{N^{\prime}}^{\mathrm{F}^{\prime}}\left(X^{\prime}, Y^{\prime}\right)=1 \Leftrightarrow R_{f_{\mathrm{p}}\left(N^{\prime}\right)}^{\mathrm{F}}\left(f_{\mathrm{e}}\left(X^{\prime}\right), f_{\mathrm{k}}\left(Y^{\prime}\right)\right)=1 .
$$

Then, a PS $\Sigma=\{$ Setup, KeyGen, Sign, Verify $\}$ for $R_{N}^{\mathrm{F}}$ can be converted into a PS $\Sigma^{\prime}=\left\{\right.$ Setup $^{\prime}$, KeyGen', Sign', Verify $\}$ for $R_{N^{\prime}}^{\mathrm{F}^{\prime}}$ as $\operatorname{Setup}^{\prime}\left(\lambda, N^{\prime}\right)=\operatorname{Setup}\left(\lambda, f_{\mathrm{p}}\left(N^{\prime}\right)\right)$, KeyGen'(msk, mpk, $\left.Y^{\prime}\right)=\operatorname{KeyGen}$ (msk, mpk, $\left.f_{\mathrm{k}}\left(Y^{\prime}\right)\right)$, Sign' $\left(\mathrm{mpk}, \mathrm{M}, X^{\prime}, \mathrm{sk}_{Y^{\prime}}, Y^{\prime}\right)=\operatorname{Sign}\left(\mathrm{mpk}, \mathrm{M}, f_{\mathrm{e}}\left(X^{\prime}\right), \mathrm{sk}_{Y^{\prime}}, f_{\mathrm{k}}\left(Y^{\prime}\right)\right)$, and Verify $(\mathrm{mpk}$, $\left.\mathrm{M}, \sigma, X^{\prime}\right)=\operatorname{Verify}\left(\mathrm{mpk}, \mathrm{M}, \sigma, f_{\mathrm{e}}\left(X^{\prime}\right)\right)$. This conversion preserves security. Namely, if $\Sigma$ is secure, so is $\Sigma^{\prime}$. This holds for adaptive-predicate and message unforgeability, selective-predicate and adaptivemessage unforgeability, and complete privacy.

Proof. Since we have $R_{N^{\prime}}^{\mathrm{F}^{\prime}}\left(X^{\prime}, Y_{0}^{\prime}\right)=R_{N^{\prime}}^{\mathrm{F}^{\prime}}\left(X^{\prime}, Y_{1}^{\prime}\right)=1 \Leftrightarrow R_{f_{\mathrm{p}}\left(N^{\prime}\right)}^{\mathrm{F}}\left(f_{\mathrm{e}}\left(X^{\prime}\right), f_{\mathrm{k}}\left(Y_{0}^{\prime}\right)\right)=R_{f_{\mathrm{p}}\left(N^{\prime}\right)}^{\mathrm{F}}\left(f_{\mathrm{e}}\left(X^{\prime}\right)\right.$, $\left.f_{\mathrm{k}}\left(Y_{1}^{\prime}\right)\right)=1$ for any $X^{\prime} \in A_{N^{\prime}}^{\prime}$ and $Y_{0}^{\prime}, Y_{1}^{\prime} \in B_{N^{\prime}}^{\prime}$, complete privacy of $\Sigma^{\prime}$ is immediately followed by that of $\Sigma$. Next we show that if $\Sigma$ is selective-attribute and adaptive-message unforgeable, so is $\Sigma^{\prime}$. Other cases can be dealt similarly. We construct a forger $\mathcal{G}$ against $\Sigma$ from a forger $\mathcal{F}$ against $\Sigma^{\prime}$. At first, $\mathcal{F}$ submits its target $X^{\prime \star}$. Then, $\mathcal{G}$ submits $f_{\mathrm{e}}\left(X^{\prime \star}\right)$ to its challenger. Then, $\operatorname{Setup}\left(\lambda, f_{\mathrm{p}}\left(N^{\prime}\right)\right)=$ $\operatorname{Setup}^{\prime}\left(\lambda, N^{\prime}\right) \rightarrow$ (mpk, msk) is run and mpk is given to $\mathcal{G}$. $\mathcal{G}$ gives mpk to $\mathcal{F}$. When $\mathcal{F}$ makes key query for $Y^{\prime} \in B_{N^{\prime}}^{\prime}, \mathcal{G}$ computes $Y=f_{\mathrm{k}}\left(Y^{\prime}\right)$ and makes a key-extraction query for its challenger. Then, $\operatorname{KeyGen}\left(\mathrm{msk}, \mathrm{mpk}, f_{\mathrm{k}}\left(Y^{\prime}\right)\right)=\operatorname{KeyGen}^{\prime}\left(\mathrm{msk}, \mathrm{mpk}, Y^{\prime}\right) \rightarrow \mathrm{sk}_{Y^{\prime}}$ is run and $\mathcal{B}$ is given $\mathrm{sk}_{Y^{\prime}}$. Then, $\mathcal{G}$ gives it to $\mathcal{F}$. Since we have $R_{f_{\mathrm{p}}\left(N^{\prime}\right)}^{\mathrm{F}}\left(f_{\mathrm{e}}\left(X^{\prime \star}\right), f_{\mathrm{k}}\left(Y^{\prime}\right)\right)=R_{N^{\prime}}^{\mathrm{F}^{\prime}}\left(X^{\prime \star}, Y^{\prime}\right)=0$, the query is valid. When $\mathcal{F}$ makes signing query for $\left(\mathrm{M}, X^{\prime}, Y^{\prime}\right)$ such that $R_{N}\left(X^{\prime}, Y^{\prime}\right)=1$, $\mathcal{G}$ submits $\left(\mathrm{M}, f_{\mathrm{e}}\left(X^{\prime}\right), f_{\mathrm{k}}\left(Y^{\prime}\right)\right)$ to its challenger. Then, KeyGen (msk, mpk, $\left.f_{\mathrm{k}}\left(Y^{\prime}\right)\right)=$ KeyGen'(msk, mpk, $\left.Y^{\prime}\right) \rightarrow$ $\mathrm{sk}_{Y^{\prime}}$ and $\operatorname{Sign}^{\prime}\left(\mathrm{mpk}, \mathrm{M}, X^{\prime}, \mathrm{sk}_{Y^{\prime}}, Y^{\prime}\right)=\operatorname{Sign}\left(\mathrm{mpk}, \mathrm{M}, f_{\mathrm{e}}\left(X^{\prime}\right), \mathrm{sk}_{Y^{\prime}}, f_{\mathrm{k}}\left(Y^{\prime}\right)\right) \rightarrow \sigma^{\prime}$ are run. Then, $\sigma^{\prime}$ is returned to $\mathcal{G}$ and $\mathcal{G}$ gives it to $\mathcal{F}$. Finally, $\mathcal{F}$ outputs its forgery $\left(\mathrm{M}^{\star}, X^{\prime \star}, \sigma^{\star}\right)$. Then, $\mathcal{G}$ outputs $\left(\mathrm{M}^{\star}, f_{\mathrm{e}}\left(X^{\prime \star}\right), \sigma^{\star}\right)$ as its forgery. We claim that $\mathcal{G}$ succeeds whenever $\mathcal{F}$ succeeds. At first, we have Verify $\left(\mathrm{mpk}, \mathrm{M}^{\star}, \sigma^{\star}, X^{\prime \star}\right)=\operatorname{Verify}\left(\mathrm{mpk}, \mathrm{M}^{\star}, \sigma^{\star}, f_{\mathrm{e}}\left(X^{\prime \star}\right)\right)$. Furthermore, all signing key queries made by $\mathcal{G}$ are of the form $\left(\mathrm{M}, f_{\mathrm{e}}\left(X^{\prime}\right), f_{\mathrm{k}}\left(Y^{\prime}\right)\right)$ where $\left(\mathrm{M}, X^{\prime}, Y^{\prime}\right)$ is submitted by $\mathcal{F}$. We have that $\mathrm{M}=$ $\mathrm{M}^{\star}, f_{\mathrm{e}}\left(X^{\prime}\right)=f_{\mathrm{e}}\left(X^{\prime \star}\right)$, and $R_{f_{\mathrm{p}}\left(N^{\prime}\right)}^{\mathrm{F}}\left(f_{\mathrm{e}}\left(X^{\prime}\right), f_{\mathrm{k}}\left(Y^{\prime}\right)\right)=1$ if and only if $\mathrm{M}=\mathrm{M}^{\star}, X^{\prime}=X^{\prime \star}$, and $R_{N^{\prime}}^{\mathrm{F}^{\prime}}\left(X^{\prime \star}, Y^{\prime}\right)=1$. Here, we used the fact that $f_{\mathrm{e}}$ is an injective map. Therefore, $\mathcal{G}$ succeeds whenever $\mathcal{F}$. This concludes the proof. 


\section{D.4 The Construction}

Here, we construct a PS scheme for relation $R_{N}^{\mathrm{KP}}$ where $N=(\bar{k},-,-,-)$ with large universe. Namely, in the following scheme, the size of attribute set that is associated with a signature is bounded by $\bar{k}$, whereas other parameters are unbounded. Furthermore, all span programs appearing in the scheme are monotone. For the sake of brevity, we assume that the message space of the following scheme to be $\{0,1\}^{\kappa}$. We also assume that the universe of attributes is $\mathcal{U}=\mathbb{Z}_{p}^{*} \backslash \mathcal{D}$ where $\mathcal{D}=\left\{D_{1}, \ldots, D_{\bar{k}}\right\} \subset \mathbb{Z}_{p}$ is a set of dummy attributes. These restrictions can be easily removed by using collision resistant hash and one can deal with message and attributes of any length.

$\operatorname{Setup}(\lambda, \bar{k})$ : It samples $h_{0}, h_{1}, \ldots, h_{\bar{k}+1}, v_{0}, v_{1}, \ldots, v_{\kappa} \stackrel{\$}{\leftarrow} \mathbb{G}$. It also samples $\alpha \stackrel{\$}{\leftarrow} \mathbb{Z}_{p}$ and outputs master public key mpk $=\left\{g, h_{0}, h_{1}, \ldots, h_{\bar{k}+1}, v_{0}, v_{1}, \ldots, v_{\kappa}, e(g, g)^{\alpha}\right\}$ and msk $=\alpha$.

In the following, we denote $\mathrm{Wa}(\mathrm{M})$ for $v_{0} \cdot \prod_{i \in\{j \in[\kappa] \mid \mathrm{M}[j]=1\}} v_{i}$ where $\mathrm{M}[j]$ is the $j$-th bit of $\mathrm{M}$. Note that $\mathrm{Wa}(\mathrm{M})$ is efficiently computable from $\mathrm{mpk}$.

KeyGen $(m s k, m p k,(\mathbf{L}, \rho))$ : The input to the algorithm is the master secret key msk, the master public key mpk, and a monotone span program $(\mathbf{L}, \rho)$. Here, $\mathbf{L} \in \mathbb{Z}_{p}^{\ell \times m}$ and $\rho$ is a map $\rho:[\ell] \rightarrow \mathcal{U}$. It chooses a vector $\mathbf{s}=(\mathbf{s}[1], \ldots, \mathbf{s}[m])^{\top}$ such that $\mathbf{s}[1]=\alpha$ and $\mathbf{s}[2], \ldots, \mathbf{s}[m] \stackrel{\$}{\leftarrow} \mathbb{Z}_{p}$ and calculates $\lambda_{i}=\mathbf{L}_{i} \cdot \mathbf{s}$ for each $i \in[\ell]$ where $\mathbf{L}_{i}$ is $i$-th row of $\mathbf{L}$. Finally, it chooses $r_{i} \stackrel{\$}{\leftarrow} \mathbb{Z}_{p}$ for all $i \in[\ell]$ and outputs private key

$$
\operatorname{sk}_{(\mathbf{L}, \rho)}=\left\{\begin{array}{lc}
D_{i, 1}=g^{\lambda_{i}} \cdot h_{0}^{r_{i}}, & D_{i, 2}=g^{r_{i}}, \\
\left\{K_{i, j}=\left(h_{1}^{-\rho(i)^{j}} h_{j+1}\right)^{r_{i}}\right\}_{j \in[1, \bar{k}]}, & \left\{K_{i, j}^{\prime}=v_{j}^{r_{i}}\right\}_{j \in[0, \kappa]}
\end{array}\right\}_{i \in[\ell]} .
$$

$\operatorname{Sign}\left(\mathrm{mpk}, \mathrm{M}, S, \mathrm{sk}_{(\mathbf{L}, \rho)},(\mathbf{L}, \rho)\right)$ : Assume that the monotone span program $(\mathbf{L}, \rho)$ associated to the private key sk $(\mathbf{L}, \rho)$ satisfies $R^{\mathrm{KP}}(S,(\mathbf{L}, \rho))=1$ and otherwise it outputs 0 . We let $I=\{i \mid \rho(i) \in$ $S\}$. Then the signing algorithm can efficiently compute reconstruction coefficients $\left\{\left(i, \mu_{i}\right)\right\}_{i \in I}=$ $\operatorname{Recon}_{\mathbf{L}, \rho}(S)$ such that $\sum_{i \in I} \mu_{i} \lambda_{i}=\alpha$. It also sets $\hat{S}=S \cup\left\{\mathrm{D}_{1}, \ldots, \mathrm{D}_{\bar{k}-|S|}\right\}$. When $|S|=\bar{k}$, $\hat{S}=S$. Then it first computes

$$
D_{i, 1}^{\prime}=D_{i, 1} \cdot \prod_{j=1}^{\bar{k}} K_{i, j}^{\mathbf{q}_{\hat{S}}[j+1]} \cdot \prod_{j^{\prime} \in\{0\} \cup\left\{j^{\prime \prime} \in[1, \kappa] \mid \mathrm{M}\left[j^{\prime \prime}\right]=1\right\}} K_{i, j^{\prime}}^{\prime}
$$

where $\mathbf{q}_{\hat{S}}=\left(\mathbf{q}_{\hat{S}}[1], \ldots, \mathbf{q}_{\hat{S}}[\bar{k}+1]\right)^{\top} \in \mathbb{Z}_{p}^{\bar{k}+1}$ is defined as a coefficient vector from $Q_{\hat{S}}[Z]=$ $\sum_{j=1}^{\bar{k}+1} \mathbf{q}_{\hat{S}}[j] \cdot Z^{j-1}=\prod_{\omega \in \hat{S}}(Z-\omega)$. Next it picks random $\tilde{r}$ and outputs the signature

$$
\sigma=\left(\sigma_{1}=\left(\prod_{i \in I}\left(D_{i, 1}^{\prime}\right)^{\mu_{i}}\right) \cdot\left(h_{0} \prod_{j=1}^{\bar{k}+1} h_{j}^{\mathbf{q}_{\hat{S}}[j]}\right)^{\tilde{r}} \cdot \mathrm{Wa}(\mathrm{M})^{\tilde{r}}, \quad \sigma_{2}=\left(\prod_{i \in I} D_{i, 2}^{-\mu_{i}}\right) \cdot g^{-\tilde{r}}\right) .
$$

Verify (mpk, $\sigma, \mathrm{M}, S)$ : To check that whether $\sigma$ is a valid signature for a message $\mathrm{M}$ with set $S$, it first checks that $\sigma \in \mathbb{G}^{2}$. Otherwise it outputs 0 . Then it checks

$$
e(g, g)^{\alpha} \stackrel{?}{=} e\left(g, \sigma_{1}\right) \cdot e\left(h_{0} \prod_{j=1}^{\bar{k}+1} h_{j}^{\mathbf{q}_{\hat{S}}[j]} \cdot \mathrm{Wa}(\mathrm{M}), \sigma_{2}\right)
$$

and outputs 1 if it holds and 0 otherwise. 
ABS with Constant-Size Signatures. The above scheme is PS scheme for relation $R^{\mathrm{KP}}$. By combining the result in Section 3 and Lemma 2, we obtain a PS scheme for relation $R^{\mathrm{DSE}}$. Furthermore, by combining the result in Section 4 and Lemma 2, we obtain the first non-monotonic ABS scheme with constant-size signatures. ${ }^{8}$

Here, we show the correctness and security of our PS scheme. Correctness of the scheme is implied by the following lemma and simple calculation. The lemma also implies privacy of the scheme since it indicates that the distribution of the signature only depends on $\mathrm{M}$ and $S$. In particular, it is completely independent from $\mathrm{sk}_{(\mathbf{L}, \rho)}$.

Lemma 3. For any $\lambda, \bar{k}, \kappa, \mathrm{M} \in\{0,1\}^{\kappa},(\mathbf{L}, \rho), S$, (mpk, msk $\leftarrow \operatorname{Setup}(\lambda, \bar{k})$, and a private key $\mathrm{sk}_{(\mathbf{L}, \rho)} \leftarrow \operatorname{KeyGen}(\mathrm{msk}, \mathrm{mpk},(\mathbf{L}, \rho))$ where $S$ is accepted by $(\mathbf{L}, \rho)$, we have that $\sigma$ generated by $\sigma \leftarrow$ $\operatorname{Sign}\left(\mathrm{mpk}, \mathrm{M}, S, \mathrm{sk}_{(\mathbf{L}, \rho)},(\mathbf{L}, \rho)\right)$ is distributed as follows:

$$
\sigma=\left(\sigma_{1}=g^{\alpha} \cdot\left(h_{0} \prod_{j=1}^{\bar{k}+1} h_{j}^{\mathbf{q}_{\hat{S}}[j]} \cdot \mathrm{Wa}(\mathrm{M})\right)^{r}, \quad \sigma_{2}=g^{-r}\right)
$$

where $r \stackrel{\$}{\leftarrow} \mathbb{Z}_{p}$.

Proof. We first observe that

$$
\begin{aligned}
& D_{i, 1}^{\prime}=g^{\lambda_{i}} \cdot h_{0}^{r_{i}} \cdot\left(\prod_{j \in[1, \bar{k}]} h_{1}^{-\rho(i)^{j} \mathbf{q}_{\hat{S}}[j+1]} h_{j+1}^{\mathbf{q}_{\hat{S}}[j+1]}\right)^{r_{i}} \cdot \mathrm{Wa}(\mathrm{M})^{r_{i}} \\
& =g^{\lambda_{i}} \cdot h_{0}^{r_{i}} \cdot h_{1}^{\left(-r_{i}\right) \cdot\left(\sum_{j \in[1, \bar{k}]} \rho(i)^{j} \mathbf{q}_{\hat{S}}[j+1]\right)} \cdot\left(\prod_{j \in[2, \bar{k}+1]} h_{j}^{\mathbf{q}_{\hat{S}}[j]}\right)^{r_{i}} \cdot \mathrm{Wa}(\mathrm{M})^{r_{i}} \\
& =g^{\lambda_{i}} \cdot\left(h_{0} \cdot \prod_{j \in[1, \bar{k}+1]} h_{j}^{\mathbf{q}_{\hat{S}}[j]}\right)^{r_{i}} \cdot \mathrm{Wa}(\mathbf{M})^{r_{i}}
\end{aligned}
$$

where we used the fact that $-\sum_{j \in[1, \bar{k}]} \rho(i)^{j} \mathbf{q}_{\hat{S}}[j+1]=\mathbf{q}_{\hat{S}}[1]$ in the last equation. This holds since if $i \in I$, we have $\rho(i) \in S \subseteq \hat{S}$ and $\sum_{j \in[1, \bar{k}+1]} \rho(i)^{j-1} \mathbf{q}_{\hat{S}}[j]=\prod_{\omega \in \hat{S}}(\rho(i)-\omega)=0$.

Next, we can see that

$$
\begin{aligned}
\sigma_{1} & =\prod_{i \in I}\left(g^{\lambda_{i}} \cdot\left(h_{0} \cdot \prod_{j \in[1, \bar{k}+1]} h_{j}^{\mathbf{q}_{\hat{S}}^{[j]}}\right)^{r_{i}} \cdot \mathrm{Wa}(\mathrm{M})\right)^{\mu_{i}} \cdot\left(h_{0} \cdot \prod_{j \in[1, \bar{k}+1]} h_{j}^{\mathbf{q}_{\hat{S}}[j]}\right)^{\tilde{r}} \cdot \mathrm{Wa}(\mathrm{M})^{\tilde{r}} \\
& =g^{\sum_{i \in I} \lambda_{i} \mu_{i}} \cdot\left(h_{0} \cdot \prod_{j \in[1, \bar{k}+1]} h_{j}^{\mathbf{q}_{\hat{S}}[j]} \cdot \mathrm{Wa}(\mathrm{M})\right)^{\tilde{r}+\sum_{i \in I} r_{i} \mu_{i}} \\
& =g^{\alpha} \cdot\left(h_{0} \prod_{j \in[1, \bar{k}+1]} h_{j}^{\mathbf{q}_{\hat{S}}[j]} \cdot \mathrm{Wa}(\mathrm{M})\right)^{r}
\end{aligned}
$$

and $\sigma_{2}=\left(\prod_{i \in I} D_{i, 2}^{-\mu_{i}}\right) \cdot g^{-\tilde{r}}=g^{-\tilde{r}-\sum_{i \in I} r_{i} \mu_{i}}=g^{-r}$ where $r=\tilde{r}+\sum_{i \in I} r_{i} \mu_{i}$. Here, $r$ is uniformly distributed over $\mathbb{Z}_{p}$ due to $\tilde{r}$ as desired.

Unforgeability of the scheme is proven under the following assumption.

\footnotetext{
${ }^{8}$ To apply Lemma 2, the map $f_{\mathrm{e}}^{\mathrm{CP} \rightarrow \mathrm{DSE}}$ should be injective. However, rigorously, it is not the case. This is because we adjust the number of columns of a matrix associated with a span program by padding zeroes in the computation of the map. This problem can be easily solved by restricting the size of matrices used in the ABS scheme to always be the same.
} 
$K$-Computational Bilinear Diffie-Hellman Exponent ( $K$-CBDHE) Assumption. We say that an adversary $\mathcal{A}$ breaks the $K$-CBDHE assumption on $\left(\mathbb{G}, \mathbb{G}_{T}\right)$ if

$$
\operatorname{Pr}\left[\mathcal{A}\left(g,\left\{g^{a^{i}}\right\}_{i \in[2 K] \backslash\{K+1\}}\right) \rightarrow g^{a^{K+1}}\right]
$$

is non-negligible and $\mathcal{A}$ runs in polynomial time where $g \stackrel{\$}{\leftarrow}, a \stackrel{\$}{\leftarrow} \mathbb{Z}_{p}$.

Before going to the security proof, we introduce following lemma proved in [45] which abstracts out core technique used in the security proof of spatial encryption scheme proposed by Boneh and Hamburg [10].

Lemma 4. ([10]) Let $\mathbb{G}$ be a multiplicative group with prime order $p$ and $g$ be its generator. Let $K, L$ be some integer bounded by polynomial of $\lambda$, a be $\mathbf{a}=\left(a, a^{2}, \ldots, a^{K}\right)^{\top} \in \mathbb{Z}_{p}^{K},\left\{w_{i}\right\}_{i=0}^{L}$ be elements in $\mathbb{Z}_{p}$, and $\left\{\mathbf{u}_{i}\right\}_{i=0}^{L}$ be vectors in $\mathbb{Z}_{p}^{K}$. We also assume that $\mathbf{t} \in \mathbb{Z}_{p}^{K}$ satisfies $\left\langle\mathbf{t}, \mathbf{u}_{0}\right\rangle \neq 0$ and $\left\langle\mathbf{t}, \mathbf{u}_{i}\right\rangle=0$ for $i \in[1, L]$. Then, there exists an PPT BHSim which takes $\left(\left\{\mathbf{u}_{i}\right\}_{i=0}^{L},\left\{w_{i}\right\}_{i=0}^{L}, \mathbf{t},\left\{g^{a^{i}}\right\}_{i \in[1,2 K] \backslash\{K+1\}}\right)$ as input and outputs $\left(g^{a^{K+1}} \cdot\left(g^{\left\langle\mathbf{u}_{0}, \mathbf{a}\right\rangle+w_{0}}\right)^{r},\left\{\left(g^{\left\langle\mathbf{u}_{i}, \mathbf{a}\right\rangle+w_{i}}\right)^{r}\right\}_{i=1}^{L}\right)$ where $r \stackrel{\$}{\leftarrow} \mathbb{Z}_{p}$.

Theorem 5. The above scheme is selective-predicate and adaptive-message unforgeable under chosen message attack if the $(\bar{k}+2)$-CBDHE assumption holds in $\mathbb{G}$.

Proof. To prove the theorem, it suffices to show that there exists a PPT $\mathcal{A}$ that solves $(\bar{k}+2)$-CBDHE problem with non-negligible probability assuming a PPT forger $\mathcal{F}$ against our scheme with non-negligible advantage. We show this by considering the following sequences of the games. In the following, let $X_{i}$ denote the probability that $\mathcal{F}$ is successful in Game $i$ and the challenger does not abort. We also let $K=\bar{k}+2$ for the simplicity of the notation.

Game $\mathbf{0}$. We define Game 0 as an experiment between the challenger and the forger $\mathcal{F}$. Let the success probability of $\mathcal{F}$ in the game be $\operatorname{Pr}\left[X_{0}\right]=\epsilon$. By the assumption, we have $\epsilon$ is non-negligible.

Game 1. In this game, we change the way to choose mpk and msk. At the outset of the game, $\mathcal{F}$ declares $S^{\star}$ which is the set she wants to be challenged upon. $\hat{S}^{\star}$ is defined as $\hat{S}^{\star}=S^{\star} \cup\left\{\mathrm{D}_{1}, \ldots, \mathrm{D}_{\bar{k}-\left|S^{\star}\right|}\right\}$. Then, the challenger picks $a, \tilde{\alpha}, \tilde{h}_{0}, \ldots, \tilde{h}_{\bar{k}+1}, \tilde{v}_{0}, \ldots, \tilde{v}_{\kappa}, \stackrel{\$}{\leftarrow} \mathbb{Z}_{p}, \phi_{0} \stackrel{\$}{\leftarrow}[-2 \kappa Q, 2 Q-1], \phi_{1}, \ldots, \phi_{\kappa} \stackrel{\$}{\leftarrow}$ $[0,2 Q-1]$ and sets msk $=a^{K+1}+\tilde{\alpha}$. Here, $Q$ is the upper bound on the number of signing queries. Next, mpk is set as

$$
\mathrm{mpk}=\left(\begin{array}{ll}
h_{0}=g^{\tilde{h}_{0}} \cdot \prod_{i=1}^{\bar{k}+1}\left(g^{a^{i}}\right)^{-\mathbf{q}_{\hat{S}^{\star}}[i]}, & h_{i}=g^{\tilde{h}_{i}} \cdot g^{a^{i}} \text { for } i \in[1, \bar{k}+1], \\
e(g, g)^{\alpha}=e\left(g^{a}, g^{a^{K}}\right) \cdot e(g, g)^{\tilde{\alpha}}, & v_{i}=\left(g^{a^{K}}\right)^{\phi_{i}} \cdot g^{\tilde{v}_{i}} \text { for } i \in[0, \kappa]
\end{array}\right)
$$

where $\mathbf{q}_{\hat{S}^{\star}}$ is defined as a coefficient vector from $Q_{\hat{S}^{\star}}[Z]=\sum_{j=1}^{\bar{k}+1} \mathbf{q}_{\hat{S}^{\star}}[j] \cdot Z^{j-1}=\prod_{\omega \in \hat{S}^{\star}}(Z-\omega)$. The rest of the game is the same as Game 0 . Since the view of $\mathcal{F}$ is completely the same as that in Game 0, we have $\operatorname{Pr}\left[X_{1}\right]=\operatorname{Pr}\left[X_{0}\right]$.

Game 2. Here, we define a function $\Phi:\{0,1\}^{\kappa} \rightarrow \mathbb{Z}_{p}$ and $\tilde{V}:\{0,1\}^{\kappa} \rightarrow \mathbb{Z}_{p}$ as

$$
\Phi(\mathrm{M})=\phi_{0}+\sum_{i \in\{j \in[\kappa] \mid \mathrm{M}[j]=1\}} \phi_{i}, \quad \tilde{V}(\mathrm{M})=\tilde{v}_{0}+\sum_{i \in\{j \in[\kappa] \mid \mathrm{M}[j]=1\}} \tilde{v}_{i} .
$$

Note that we have $\mathrm{Wa}(\mathrm{M})=\left(g^{a^{K}}\right)^{\Phi(\mathrm{M})} \cdot g^{\tilde{V}(\mathrm{M})}$ by this definition. In this game, the challenger aborts if $\mathcal{F}$ makes signing query for $\left(\mathrm{M}, S^{\star},(\mathbf{L}, \rho)\right)$ such that $\Phi(\mathrm{M})=0$ and $R_{N}\left(S^{\star},(\mathbf{L}, \rho)\right)=1$. 
The challenger also aborts if the final output $\left(\mathrm{M}^{\star}, \sigma^{\star}\right)$ of $\mathcal{F}$ satisfies $\Phi\left(\mathrm{M}^{\star}\right) \neq 0$. By the same argument as the security proof of the Waters' identity-based encryption scheme [42], we have that $\operatorname{Pr}\left[X_{2}\right] \geq \operatorname{Pr}\left[X_{1}\right] /(32(\kappa+1) Q)$.

Finally, we replace the challenger in Game 2 with an algorithm $\mathcal{A}$ that solves CBDHE problem with probability $\operatorname{Pr}\left[X_{2}\right]$ which is non-negligible assuming $\operatorname{Pr}\left[X_{0}\right]$ is non-negligible. We describe the description of $\mathcal{A}$ in the following.

Set-up of the Public Keys. Given the problem instance $\left(g,\left\{g^{a^{i}}\right\}_{i \in[1,2 K] \backslash\{K+1\}}\right)$ and $S^{\star}$ from $\mathcal{F}, \mathcal{A}$ picks $\tilde{\alpha}, \tilde{h}_{0}, \ldots, \tilde{h}_{\bar{k}+1}, \tilde{v}_{0}, \ldots, \tilde{v}_{\kappa}, \stackrel{\$}{\leftarrow} \mathbb{Z}_{p}, \phi_{0} \stackrel{\$}{\leftarrow}[-2 \kappa Q, 2 Q-1], \phi_{1}, \ldots, \phi_{\kappa} \stackrel{\$}{\leftarrow}[0,2 Q-1]$, sets mpk as Equation (9), and gives it to $\mathcal{F}$. This step can be efficiently done only using problem instance, in particular, without knowing $a$ or $g^{a^{K+1}}$.

Answering Private Key Queries. Throughout the game, $\mathcal{F}$ may ask for a private key for $(\mathbf{L}, \rho)$. Let $\mathbf{L}$ be an $\ell \times m$ matrix and $I$ be $I=\left\{i \in[1, \ell] \mid \rho(i) \in \hat{S}^{\star}\right\}$. Since $\mathbf{L}$ does not accept $\hat{S}^{\star}$, we have $\mathbf{e}_{1} \notin \operatorname{span}\left(\mathbf{L}_{I}^{\top}\right)$ where $\mathbf{L}_{I}$ is the sub-matrix of $(\mathbf{L}, \rho)$ formed by rows corresponding to index $i$ such that $i \in I$. Hence, due to the proposition 11 in [25], we have that there must exists an efficiently computable vector $\mathbf{z} \in \mathbb{Z}_{p}^{m}$ such that $\left\langle\mathbf{e}_{1}, \mathbf{z}\right\rangle=1$ and $\mathbf{L}_{I} \cdot \mathbf{z}=\mathbf{0}$. Now $\mathcal{A}$ picks $\mathbf{w}[2], \ldots, \mathbf{w}[m] \stackrel{\$}{\leftarrow} \mathbb{Z}_{p}$ and implicitly defines $\mathbf{s}=(\mathbf{s}[1], \ldots, \mathbf{s}[m])^{\top}=\alpha \mathbf{z}+\mathbf{w}$ where $\mathbf{w}=(0, \mathbf{w}[2], \ldots, \mathbf{w}[m])^{\top}$. Note that we have that $\mathbf{s}[1]=\alpha$ and that $\mathbf{s}[2], \ldots, \mathbf{s}[m] \in \mathbb{Z}_{p}$ are uniformly distributed, as required in Definition 1 . Then $\mathcal{F}$ implicitly defines $\lambda_{i}=\mathbf{L}_{i} \cdot \mathbf{s}$ where $\mathbf{L}_{i}$ is the $i$-th row of $\mathbf{L}$. Then $\mathcal{A}$ computes $\left(D_{i, 1}, D_{i, 2},\left\{K_{i, j}\right\}_{j \in[1, \bar{k}]},\left\{K_{i, j}^{\prime}\right\}_{j \in[0, \kappa]}\right)$ for $i \in[1, \ell]$ as follows.

- For $i \in I$, we have that $\lambda_{i}=\mathbf{L}_{i} \cdot \mathbf{s}=\mathbf{L}_{i} \cdot \mathbf{w}$ holds since $\mathbf{L}_{i} \cdot \mathbf{z}=0$ and thus $\lambda_{i}$ can be efficiently computed by $\mathcal{A}$. In this case, $\mathcal{A}$ picks $r_{i} \stackrel{\$}{\leftarrow} \mathbb{Z}_{p}$ and computes $D_{i, 1}=g^{\lambda_{i}} \cdot h_{0}^{r_{i}}, D_{i, 2}=g^{r_{i}}$, $\left\{K_{i, j}=\left(h_{1}^{-\rho(i)^{j}} h_{j+1}\right)^{r_{i}}\right\}_{j \in[1, \bar{k}]}$, and $\left\{K_{i, j}^{\prime}=v_{j}^{r_{i}}\right\}_{j \in[0, \kappa]}$.

- For $i \notin I$, we have that $\lambda_{i}=\alpha \mathbf{L}_{i} \cdot \mathbf{z}+\mathbf{L}_{i} \cdot \mathbf{w}=\mu_{1} a^{K+1}+\mu_{2}$ where $\mu_{1}=\mathbf{L}_{i} \cdot \mathbf{z}$ and $\mu_{2}=\mathbf{L}_{i} \cdot(\tilde{\alpha} \mathbf{z}+\mathbf{w})$. $\lambda_{i}$ is known to $\mathcal{A}$ if $\mu_{1}=0$ and such a case can be dealt with as the same as the case of $i \in I$. Thus we assume that $\mu_{1} \neq 0$ in the following. Since $\mathcal{A}$ does not know $\lambda_{i}$, we have to rely on Lemma 4 in order to answer the query.

$\mathcal{A}$ sets $\mathbf{u}_{0}=\left(-\mathbf{q}_{\hat{S}^{\star}}^{\top}, 0\right)^{\top} \in \mathbb{Z}_{p}^{K}, w_{0}=\tilde{h}_{0}, \mathbf{u}_{1}=\mathbf{0}_{K} \in \mathbb{Z}_{p}^{K}, w_{1}=1, \mathbf{u}_{j+1}=-\rho(i)^{j} \mathbf{e}_{1}+$ $\mathbf{e}_{j+1} \in \mathbb{Z}_{p}^{K}$ and $w_{j+1}=-\rho(i)^{j} \tilde{h}_{1}+\tilde{h}_{j+1}$ for $j \in[1, \bar{k}]$, and $\mathbf{u}_{j^{\prime}+\bar{k}+2}=\phi_{j^{\prime}} \mathbf{e}_{K} \in \mathbb{Z}_{p}^{K}$ and $w_{j^{\prime}+\bar{k}+2}=\tilde{v}_{j^{\prime}}$ for $j^{\prime} \in[0, \kappa]$. It also sets $\mathbf{t}=\left(1, \rho(i), \ldots, \rho(i)^{K-1}, 0\right) \in \mathbb{Z}_{p}^{K}$. We can see that $\left\langle\mathbf{u}_{0}, \mathbf{t}\right\rangle=-\prod_{\omega \in \hat{S}^{\star}}(\rho(i)-\omega) \neq 0$ since $\rho(i) \notin \hat{S}^{\star}$ and $\left\langle\mathbf{u}_{j}, \mathbf{t}\right\rangle=0$ for $j \in[1, \bar{k}+\kappa+2]$.

Then $\mathcal{A}$ runs $\operatorname{BHSim}\left(\left\{\mathbf{u}_{i}\right\}_{i=0}^{\bar{k}+\kappa+2},\left\{w_{i}\right\}_{i=0}^{\bar{k}+\kappa+2}, \mathbf{t},\left\{g^{a^{i}}\right\}_{i \in[1,2 K] \backslash\{K+1\}}\right) \rightarrow\left(g^{a^{K+1}} \cdot\left(g^{\left\langle\mathbf{u}_{0}, \mathbf{a}\right\rangle+w_{0}}\right)^{\hat{r}}\right.$, $\left.\left\{\left(g^{\left\langle\mathbf{u}_{i}, \mathbf{a}\right\rangle+w_{i}}\right)^{\hat{r}}\right\}_{i=1}^{\bar{k}+\kappa+2}\right)$ where $\hat{r} \stackrel{\$}{\leftarrow} \mathbb{Z}_{p}$ due to Lemma 4. We have that $\hat{D}_{i, 1}:=g^{a^{K+1}} \cdot\left(g^{\left\langle\mathbf{u}_{0}, \mathbf{a}\right\rangle+w_{0}}\right)^{\hat{r}}=$ $g^{a^{K+1}} \cdot\left(g^{\tilde{h}_{0}} \cdot \prod_{i=1}^{\bar{k}+1}\left(g^{a^{i}}\right)^{-\mathbf{q}_{\hat{S}^{\star}}[i]}\right)^{\hat{r}}=g^{a^{K+1}} \cdot h_{0}^{\hat{r}}, \hat{D}_{i, 2}:=\left(g^{\left\langle\mathbf{u}_{1}, \mathbf{a}\right\rangle+w_{1}}\right)^{\hat{r}}=g^{\hat{r}}, \hat{K}_{i, j}:=\left(g^{\left\langle\mathbf{u}_{j+1}, \mathbf{a}\right\rangle+w_{j+1}}\right)^{\hat{r}}$ $=\left(\left(g^{a}\right)^{-\rho(i)^{j}} \cdot g^{a^{j+1}} \cdot g^{-\rho(i)^{j} \tilde{h}_{1}+\tilde{h}_{j+1}}\right)^{\hat{r}}=\left(h_{1}^{-\rho(i)^{j}} h_{j+1}\right)^{\hat{r}}$ for $j \in[1, \bar{k}]$, and also we have $\hat{K}_{i, j^{\prime}}^{\prime}=$ $\left(g^{\left\langle\mathbf{u}_{j^{\prime}+\bar{k}+2}, \mathbf{a}\right\rangle+w_{j^{\prime}+\bar{k}+2}}\right)^{\hat{r}}=\left(\left(g^{a^{K}}\right)^{\phi_{j^{\prime}}} \cdot g^{\tilde{v}_{j^{\prime}}}\right)^{\hat{r}}=v_{j^{\prime}}^{\hat{r}}$ for $j^{\prime} \in[0, \kappa]$.

Finally, $\mathcal{A}$ sets $D_{i, 1}=\hat{d}_{i, 1}^{\mu_{1}} \cdot g^{\mu_{2}}=g^{\alpha} \cdot h_{0}^{r_{i}}, D_{i, 2}=\hat{D}_{i, 1}^{\mu_{1}}=g^{r_{i}}, K_{i, j}=\hat{K}_{i, j}^{\mu_{1}}=\left(h_{1}^{-\rho(i)^{j}} \cdot h_{j+1}\right)^{r_{i}}$ for $j \in[1, \bar{k}]$, and $K_{i, j^{\prime}}^{\prime}=\left(\hat{K}_{i, j^{\prime}}^{\prime}\right)^{\mu_{i}}=v_{j^{\prime}}^{r_{i}}$ for $j^{\prime} \in[0, \kappa]$ where $r_{i}=\mu_{1} \hat{r}$. Since $\mu_{1} \neq 0$ and $\hat{r}$ is uniformly distributed over $\mathbb{Z}_{p}, r_{i}$ is also uniformly distributed over $\mathbb{Z}_{p}$ as desired.

Finally, $\mathcal{A}$ gives sk $_{(\mathbf{L}, \rho)}=\left\{D_{i, 1}, D_{i, 2},\left\{K_{i, j}\right\}_{j \in[1, \bar{k}]},\left\{K_{i, j}^{\prime}\right\}_{j \in[0, \kappa]}\right\}_{i \in[1, \ell]}$ to $\mathcal{F}$. 
Answering Signing Queries. When $\mathcal{F}$ makes a signing query to $(\mathrm{M}, S,(\mathbf{L}, \rho)), \mathcal{A}$ checks whether $R^{\mathrm{KP}}(S,(\mathbf{L}, \rho))=0$ and returns $\perp$ if it holds. Otherwise, it answers the signing query as follows. There are two cases to consider.

- In case of $S \neq S^{\star}$, we have that $\hat{S} \neq \hat{S}^{\star}$ where $\hat{S}=S \cup\left\{\mathrm{D}_{1}, \ldots, \mathrm{D}_{\bar{k}-|S|}\right\}$. Since $\hat{S} \neq \hat{S}^{\star}$ and $|\hat{S}|=\left|\hat{S}^{\star}\right|$, there exists $U \in \mathcal{U} \cup \mathcal{D}=\mathbb{Z}_{p}^{*}$ such that $U \notin \hat{S}^{\star}$ and $U \in \hat{S}$. Then $\mathcal{A}$ sets a monotone span program $\left(\mathbf{L}^{\prime}, \rho^{\prime}\right)$ over $\mathcal{U} \cup \mathcal{D}$ such that $\mathbf{L}^{\prime}=(1) \in \mathbb{Z}_{p}^{1 \times 1}$ and $\rho^{\prime}(1)=\mathrm{U}$. The monotone span program accepts a set iff it includes $U$. So we have that $\left(\mathbf{L}^{\prime}, \rho^{\prime}\right)$ accepts $\hat{S}$ while it does not accept $\hat{S}^{\star}$. Then $\mathcal{A}$ can generates a private key $\operatorname{sk}_{\left(\mathbf{L}^{\prime}, \rho^{\prime}\right)}=\left\{g^{\alpha} \cdot h_{0}^{r}, g^{r},\left\{\left(h_{1}^{-U^{j}} h_{j+1}\right)^{r}\right\}_{j \in[1, \bar{k}]},\left\{v_{j^{\prime}}^{r}\right\}_{j^{\prime} \in[0, \kappa]}\right\}$ for $\left(\mathbf{L}^{\prime}, \rho^{\prime}\right)$ such that $r \stackrel{\$}{\leftarrow} \mathbb{Z}_{p}$ by the same way as answering private key queries. Note that such a monotone span program is not considered to be valid one in the real system when $U \in \mathcal{D}$. We consider such a key only in the security proof. Then $\mathcal{A}$ runs $\sigma \leftarrow \operatorname{Sign}\left(\mathrm{mpk}, \mathrm{M}, S, \mathrm{sk}_{\left(\mathbf{L}^{\prime}, \rho^{\prime}\right)},\left(\mathbf{L}^{\prime}, \rho^{\prime}\right)\right)$ and returns $\sigma$ to $\mathcal{F}$. Due to the perfect privacy of the scheme, the distribution of $\sigma$ is completely the same as honestly generated one.

- Next we consider the case of $S^{\star}=S$. First assume that $\Phi(\mathrm{M}) \neq 0$ since otherwise $\mathcal{A}$ aborts. $\mathcal{A}$ picks $\hat{r} \stackrel{s}{\leftarrow} \mathbb{Z}_{p}$ and implicitly sets $r=-a / \Phi(\mathrm{M})+\hat{r}$. Then $\sigma=\left(\sigma_{1}, \sigma_{2}\right)$ is set as

$$
\begin{aligned}
& \sigma_{1}=g^{\alpha} \cdot\left(h_{0} \prod_{j \in[1, \bar{k}+1]} h_{j}^{\mathbf{q}_{\hat{S}^{\star}}[j]} \cdot \mathrm{Wa}(\mathrm{M})\right)^{r} \\
& =g^{a^{K+1}+\tilde{\alpha}} \cdot\left(g^{\tilde{h}_{0}+\sum_{i \in[1, \bar{k}+1]} \mathbf{q}_{\hat{S}^{\star}}[i] \tilde{h}_{i}} \cdot\left(g^{a^{K}}\right)^{\Phi(\mathrm{M})} g^{\tilde{V}(\mathrm{M})}\right)^{-a / \Phi(\mathrm{M})+\hat{r}} \\
& =\left(g^{a}\right)^{-\tilde{h} / \Phi(\mathrm{M})} \cdot\left(g^{a^{K}}\right)^{\Phi(\mathrm{M}) \hat{r}} \cdot g^{\tilde{\alpha}+\hat{r} \tilde{h}}
\end{aligned}
$$

and $\sigma_{2}=\left(g^{a}\right)^{-1 / \Phi(\mathrm{M})} \cdot g^{\hat{r}}$ where $\tilde{h}=\tilde{h}_{0}+\sum_{i \in[1, \bar{k}+1]} \mathbf{q}_{\hat{S}^{\star}}[i] \tilde{h}_{i}+\tilde{V}(\mathrm{M})$. In the Equation (10), a problematic term $g^{a^{K+1}}$ cancels out. Thus $\mathcal{A}$ can efficiently compute $\sigma_{1}$ and $\sigma_{2}$ from the problem instance. Furthermore, since $r$ is uniformly distributed over $\mathbb{Z}_{p}, \sigma$ is correctly distributed by Lemma 3.

Extracting the Answer to the Problem from Forgery. At the last of the game, $\mathcal{F}$ outputs a forgery $\left(\mathrm{M}^{\star}, \sigma^{\star}=\left(\sigma_{1}^{\star}, \sigma_{2}^{\star}\right)\right)$ for the set $S^{\star}$. $\mathcal{A}$ aborts if $\Phi\left(\mathrm{M}^{\star}\right) \neq 0$ or Verify $\left(\mathrm{mpk}, \sigma^{\star}, \mathrm{M}^{\star}, S^{\star}\right)=0$. Otherwise, $\mathcal{A}$ can extract the answer to the CBDHE assumption as follows. Since Verify $\left(\mathrm{mpk}, \sigma^{\star}, \mathrm{M}^{\star}, S^{\star}\right)=1$, we have that $\sigma_{2}^{\star}=g^{-r}$ and

$$
\begin{aligned}
\sigma_{1}^{\star} & =g^{\alpha} \cdot\left(h_{0} \prod_{j \in[1, \bar{k}+1]} h_{j}^{\mathbf{q}_{\hat{S}^{\star}}[j]} \cdot \mathrm{Wa}\left(\mathrm{M}^{\star}\right)\right)^{r} \\
& =g^{a^{K+1}+\tilde{\alpha}} \cdot\left(g^{r}\right)^{\tilde{h}_{0}+\left(\sum_{j \in[1, \bar{k}+1]} \mathbf{q}_{\hat{S}^{\star}}[j] \cdot \tilde{h}_{j}\right)+\tilde{V}\left(\mathrm{M}^{\star}\right)}
\end{aligned}
$$

for some $r \in \mathbb{Z}_{p}$. The second equation in the above follows from the fact that $\Phi\left(\mathrm{M}^{\star}\right)=0$. Thus $\mathcal{A}$ can extract $g^{a^{K+1}}$ by computing $\sigma_{1}^{\star} \cdot g^{-\tilde{\alpha}} \cdot\left(\sigma_{2}^{\star}\right)^{\tilde{h}_{0}+\left(\sum_{j \in[1, \bar{k}+1]} \mathbf{q}_{\hat{S}^{\star}}[j] \cdot \tilde{h}_{j}\right)+\tilde{V}\left(\mathrm{M}^{\star}\right)}=g^{a^{K+1}}$.

Since the view of $\mathcal{F}$ is completely the same as that in Game 2, the success probability of $\mathcal{A}$ is $\operatorname{Pr}\left[X_{2}\right]$ which is non-negligible by the assumption. 


\section{Contents}

1 Introduction $\quad 2$

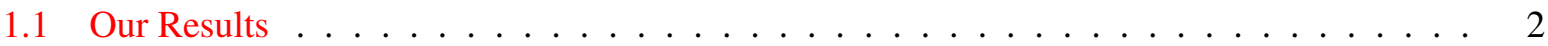

1.2 Related Works . . . . . . . . . . . . . . . . . . . . . . . 4

2 Preliminaries $\quad 5$

2.1 Definition of Predicate Encryption . . . . . . . . . . . . . . . 5

2.2 (Arithmetic) Span Program, ABE, and Doubly Spatial Encryption . . . . . . . . . . . 6

2.3 Embedding Lemma for $\mathrm{PE} \ldots \ldots \ldots \ldots \ldots$

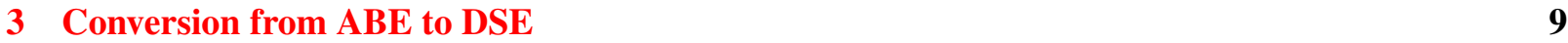

3.1 The Conversion . . . . . . . . . . . . . . . . . . . . . . 9

3.2 Correctness of the Conversion $\ldots \ldots \ldots \ldots \ldots$

4 From DSE to Non-Monotonic ABE 12

4.1 The Conversion . . . . . . . . . . . . . . . . . . . . . 13

4.2 Correctness of the Conversion . . . . . . . . . . . . . . . . . 14

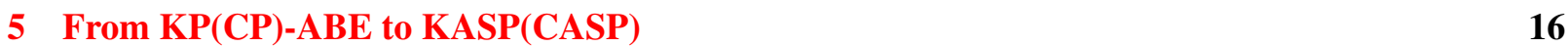

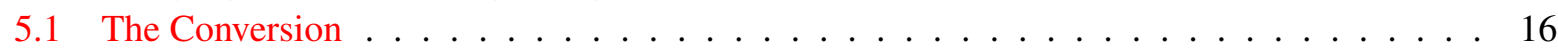

5.2 Correctness of the Conversion $\ldots \ldots \ldots \ldots \ldots \ldots \ldots$

6 Implications of Our Result $\quad 18$

7 Application to Attribute-Based Signature $\quad 21$

$\begin{array}{ll}\text { References } & 22\end{array}$

$\begin{array}{lll}\text { A Proof of Lemma } 1 & 23\end{array}$

B Key Delegation Algorithm $\quad 24$

C From K(C)ASP to KP(CP)-ABE $\quad 27$

C.1 The Conversion . . . . . . . . . . . . . . . . . . . . . . 27

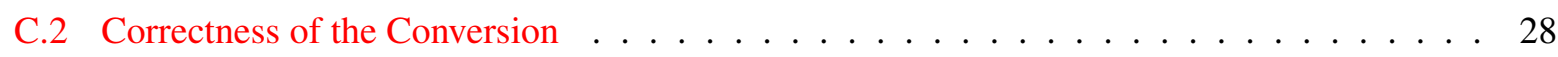

$\begin{array}{ll}\text { D Our Attribute-Based Signature Scheme } & 28\end{array}$

D.1 Predicate Signature . . . . . . . . . . . . . . . . . . . . . . . . . 29

D.2 Linear Secret Sharing Scheme . . . . . . . . . . . . . . . . . . . . . . . . 29

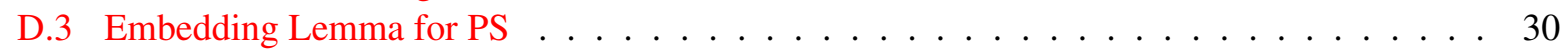

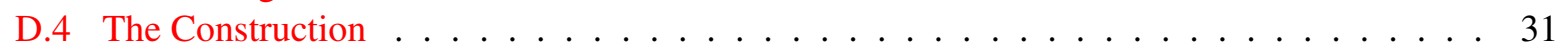

